\title{
The angiocrine Rspondin3 instructs interstitial macrophage transition via metabolic-epigenetic reprogramming and resolves inflammatory injury
}

\author{
Bisheng Zhou ${ }^{1,2}$, Lissette Magana ${ }^{1,2}$, Zhigang Hong ${ }^{1,2}$, Long Shuang Huang ${ }^{1,2}$, \\ Sreeparna Chakraborty 1,2, Yoshikazu Tsukasaki ${ }^{1,2}$, Cary Huang ${ }^{1,2}$, Li Wang 1,2, Anke Di ${ }^{1,2}$, \\ Balaji Ganesh ${ }^{3}$, Xiaopei Gao ${ }^{1,2}$, Jalees Rehman ${ }^{1,2,4} \bowtie$ and Asrar B. Malik ${ }^{1,2} \bowtie$
}

\begin{abstract}
Macrophages demonstrate remarkable plasticity that is essential for host defense and tissue repair. The tissue niche imprints macrophage identity, phenotype and function. The role of vascular endothelial signals in tailoring the phenotype and function of tissue macrophages remains unknown. The lung is a highly vascularized organ and replete with a large population of resident macrophages. We found that, in response to inflammatory injury, lung endothelial cells release the Wnt signaling modulator Rspondin3, which activates $\beta$-catenin signaling in lung interstitial macrophages and increases mitochondrial respiration by glutaminolysis. The generated tricarboxylic acid cycle intermediate $\alpha$-ketoglutarate, in turn, serves as the cofactor for the epigenetic regulator TET2 to catalyze DNA hydroxymethylation. Notably, endothelial-specific deletion of Rspondin3 prevented the formation of anti-inflammatory interstitial macrophages in endotoxemic mice and induced unchecked severe inflammatory injury. Thus, the angiocrine-metabolic-epigenetic signaling axis specified by the endothelium is essential for reprogramming interstitial macrophages and dampening inflammatory injury.
\end{abstract}

M acrophages in all tissues exhibit remarkable phenotypic plasticity, characterized by transitioning into distinct phenotypes with specific functions in response to microenvironmental cues ${ }^{1,2}$. Infection and injury drive the generation of pro-inflammatory phenotypes, whereas tissue niche signals can induce the switch of tissue macrophages toward anti-inflammatory and pro-reparative phenotypes to facilitate the resolution of inflammation $^{1,3}$. Thus, the orchestration of pro- and anti-inflammatory macrophage phenotypes governs the fate of organs during inflammation and injury ${ }^{1,3}$. In the lung, deregulation of macrophages is a leading cause of unrestrained inflammation to bacterial and viral infection and is a critical factor in the pathogenesis of acute lung injury (ALI) and acute respiratory distress syndrome, including its most severe manifestations involving cytokine storms that have been described in COVID-19 (refs. ${ }^{4,5}$ ).

Macrophage reprogramming requires tight regulation of gene expression governed by epigenetic programs and transcriptional regulation ${ }^{6,7}$. Also, studies have identified that metabolic adaptation is both a critical hallmark and prerequisite for the macrophage phenotype switch ${ }^{8,9}$. Local microenvironmental cues generated by tissue cells are increasingly recognized as critical determinants of resident macrophage identity, phenotype and function ${ }^{10-12}$. Resident macrophages are highly heterogeneous and unique as they occupy distinct tissue niches and hence exhibit the phenotype and function that is imprinted by niche-derived signals that trigger specific differentiation programs ${ }^{10,13,14}$.

Macrophages represent the most abundant immune cells in the healthy lung, consisting of two types of tissue-resident macrophages that are characterized by their localization: alveolar macrophages (AMs) that populate alveoli and airways and interstitial macrophages (IMs) that reside in lung parenchyma ${ }^{13}$. Lung IMs are less well understood but findings suggest that they are critical for maintaining lung homeostasis ${ }^{15,16}$. The vascular endothelial cells (ECs) lining all blood vessels serve as conduits for blood and tissue nutrient delivery but also constitute a niche for lung macrophages ${ }^{17}$. How the lung endothelial niche regulates lung macrophage plasticity is not known. In the present study, we found that the vascular endothelial niche was essential for lung IM reprogramming through the activation of a metabolic-epigenetic signaling axis. ECs release the angiocrine mediator Rspondin3 to induce metabolic and epigenetic reprogramming of IMs toward an anti-inflammatory phenotype via activation of the Wnt signaling pathway and were shown to mitigate inflammatory lung injury.

\section{Results}

Endothelial cells instruct macrophage phenotypic transition via angiocrine signaling. We first analyzed the paracrine factors released by ECs that could regulate macrophage transition (Extended Data Fig. 1a). We isolated lung ECs and collected conditioned medium from either LPS-activated ECs or control ECs, which was added to bone-marrow-derived macrophages (BMDMs). After $24 \mathrm{~h}$, BMDMs were collected for flow cytometry analysis. Macrophages were first gated by $\mathrm{CD} 11 \mathrm{~b}^{+} \mathrm{F} 4 / 80^{+} \mathrm{CD}^{+} 4^{+}$(gating strategy shown in Supplementary Fig. 1i) and the expression of anti-inflammatory markers (CD206, CD301, arginase 1 and interleukin (IL)-10) as well as pro-inflammatory macrophage markers (CD86, CD80, tumor necrosis factor (TNF) and inducible nitric oxide synthase (iNOS)) was analyzed. We observed that EC-conditioned medium significantly induced the expression of anti-inflammatory markers while suppressing pro-inflammatory markers and this trend was

'Department of Pharmacology and Regenerative Medicine, University of Illinois College of Medicine, Chicago, IL, USA. ${ }^{2}$ Center for Lung and Vascular Biology, University of Illinois College of Medicine, Chicago, IL, USA. ${ }^{3}$ Research Resources Center, University of Illinois, Chicago, IL, USA. ${ }^{4}$ Division of

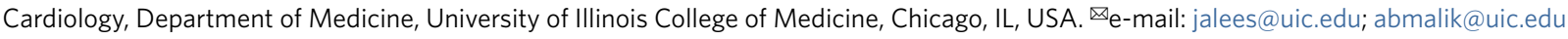


markedly augmented in ECs activated by the bacterial endotoxin lipopolysaccharide (LPS) (Fig. 1a). We also quantified the percentages of 'M1-like' (defined as 'CD86 ${ }^{\text {hi }} \mathrm{CD} 206^{\text {lo' }}$ ) and 'M2-like' (defined as 'CD206 ${ }^{\text {hi }} \mathrm{CD} 86^{\mathrm{lo}}$ ) macrophages ${ }^{18}$ and found that ECconditioned medium shifted the balance of macrophage populations toward an M2-like phenotype (Extended Data Fig. 1c,d). We then carried out a secretome analysis to identify candidate proteins mediating the paracrine EC effects on macrophage phenotype transition. We found that several proteins were released by ECs following LPS activation (Supplementary Table 1), with the Wnt signaling activator Rspondin 3 clearly ranked as the top secreted EC protein (Fig. 1b) and the release of Rspondin 3 was validated by quantitative ELISA (Fig. 1c) but without change on Rspo3 messenger RNA levels (Extended Data Fig. 1b). This suggested that angiocrine signals such as Rspondin 3 could be potential regulators for macrophage phenotype transition.

Rspondin3 mediates interstitial macrophage phenotypic transition and prevents inflammatory lung injury. To determine the role of Rspondin3 in regulating macrophage phenotype transition, BMDMs were stimulated with recombinant Rspondin3 protein $\left(40 \mathrm{ng} \mathrm{ml}^{-1}\right)$ and LPS $\left(100 \mathrm{ng} \mathrm{ml}^{-1}\right)$ alone or in combination. Flow cytometry analysis demonstrated that Rspondin 3 increased the expression of anti-inflammatory markers while concomitantly reducing pro-inflammatory markers in macrophages, whereas LPS strongly induced pro-inflammatory markers and reduced anti-inflammatory markers; crucially, Rspondin3 prevented the generation of LPS-induced pro-inflammatory markers (Fig. 1d and Extended Data Fig. 1e). We also found that Rspondin3 induced the release of anti-inflammatory cytokines such as IL-10 while reducing the release of pro-inflammatory cytokines such as IL-1 $\beta$ in macrophages (Fig. 1e). We observed increased expression of multiple anti-inflammatory marker genes (Mrc1, Arg1, Chil3, Retnla and Pparg and Il10) and decreased expression of pro-inflammatory marker genes (Cd86, Il1b, Tnf, Cxcl1, Il6 and Nos2) induced by Rspondin3 (Fig. If and Extended Data Fig. 1f), underscoring the crucial role of Rspondin 3 in promoting a macrophage shift toward an anti-inflammatory phenotype. Furthermore, we induced endotoxemia in wild-type (WT) mice and VE-cadherin-CreERT2 $2^{+} ; R s p o 3^{\mathrm{f} / \mathrm{fl}}$ mice (herein called $R s p o 3^{\mathrm{EC}-1-}$ mice) and isolated lung ECs from these mice as well as nonendotoxemic control mice (Supplementary Fig. 1). Conditioned medium collected from these ECs showed that Rspondin3 deficiency, specifically in EC medium, prevented the induction of anti-inflammatory markers and suppression of pro-inflammatory markers in macrophages (Extended Data Fig. 2a); however, EC-macrophage contact is dispensable for these effects (Extended Data Fig. 2b). This demonstrated the critical role of angiocrine Rspondin3 in mediating the effects of lung ECs on macrophage phenotype transition.

The mouse lung has two distinct resident macrophage populations, including AMs and IMs, which are involved in the regulation of lung homeostasis as well as lung injury ${ }^{19}$. In addition, other myeloid cells, such as monocytes (Mo), including $\mathrm{Ly}_{6 \mathrm{C}}{ }^{+}$Mo and Ly6C $^{-} \mathrm{Mo}$, neutrophils, dendritic cells (DCs), including $\mathrm{CD}_{103}{ }^{+}$ DCs, plasmacytoid DCs and CD11 $\mathrm{b}^{+}$DCs and eosinophils also reside in lungs ${ }^{20}$. We assessed AMs, IMs and other myeloid lung populations using cytometry by time-of-flight mass spectrometry (CyTOF), which enabled the detection of more than 30 surface markers and intracellular molecules simultaneously, using metal-labeled antibodies (Supplementary Table 4) with a myeloid cell-gating strategy (Fig. 2a). The absolute number of AMs, IMs, Ly6C $^{+}$Mo, Ly6C - Mo, neutrophils, CD103+ DCs, plasmacytoid DCs, CD $11 \mathrm{~b}^{+}$DCs and eosinophils in WT and Rspo $^{\mathrm{EC}-1-}$ mice with or without intravenous (i.v.) administration of Rspondin3 under basal and sublethal LPS challenge conditions $\left(12 \mathrm{mg} \mathrm{kg}^{-1}\right.$ intraperitoneally (i.p.) for $24 \mathrm{~h}$ or $48 \mathrm{~h}$ ) are shown in Fig. $2 \mathrm{~b}$ and Extended Data Fig. 3a. IMs expanded twofold to threefold in response to endotoxemia at $24 \mathrm{~h}$ and $48 \mathrm{~h}$ in WT mice, whereas the number of IMs did not change in Rspo $3^{\mathrm{EC}-/-}$ mice (Fig. 2b). The results thus show that endothelial Rspondin3 is a prerequisite for the expansion of IMs in response to endotoxemia (Fig. 2b). In addition, i.v. Rspondin3 was able to partially restore endotoxemia-induced IM expansion in Rspo3 $3^{\mathrm{EC}-/-}$ mice (Fig. 2b). However, the number of AMs in WT and Rspo $3^{\mathrm{EC}-1-}$ mice remained the same during the basal state and post-endotoxemia and i.v. Rspondin3 did not change AM numbers (Fig. 2b). The other myeloid populations, monocytes, DCs and eosinophils were also not affected by Rspondin3 (Extended Data Fig. 3a). Neutrophils in WT and Rspo $3^{\mathrm{EC}-1-}$ mice remained the same in basal conditions and $48 \mathrm{~h}$ post-LPS challenge but there were more neutrophils in Rspo $3^{\mathrm{EC}-/-}$ mice $24 \mathrm{~h}$ post-LPS (Extended Data Fig. 3a), likely reflecting exacerbated inflammatory injury at this time point in the absence of endothelial Rspondin3. Moreover, Rspondin3 activates Wnt signaling in IMs but not other lung myeloid cells (Extended Data Fig. 3d).

The levels of anti-inflammatory markers (CD206, CD301, RELM- $\alpha$, arginase 1 and IL-10) and pro-inflammatory markers

Fig. 1 | ECs instruct macrophage phenotype transition via the angiocrine factor Rspondin3. a, Levels of anti-inflammatory markers (CD206, CD301, arginase 1 and IL-10) and pro-inflammatory markers (CD86, CD80, TNF and iNOS) in BMDMs incubated with control medium, normal murine lung EC-conditioned medium (EC) or LPS-activated murine lung EC-conditioned medium (activated EC) as measured by flow cytometry. Representative overlaid flow cytometry histograms showing mean fluorescence intensity (MFI) (left) and quantified data from three independent experiments with $n=3$ samples per group (mean \pm s.d.) (right). Statistical significance was determined by two-way analysis of variance (ANOVA) with Dunnett's multiple comparisons test using GraphPad Prism and individual $P$ values (left to right) are: CD206 ( ${ }^{\star} P=0.0249$, ${ }^{\star \star \star \star} P<0.0001$ ); CD301 ( ${ }^{\star \star \star \star} P<0.0001$, ${ }^{\star \star \star \star} P<0.0001$ ); arginase 1

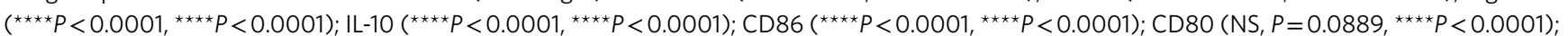
TNF ( $\left.{ }^{\star \star \star \star} P<0.0001,{ }^{\star \star \star \star} P<0.0001\right)$; and iNOS ( $\left.{ }^{\star \star \star \star} P<0.0001,{ }^{\star \star \star \star} P<0.0001\right)$. NS, not significant. $\mathbf{b}$, Top-ranked secreted proteins identified by a proteomics-based secretome assay using conditioned medium collected from LPS-activated murine lung ECs (normalized to total spectra). c, ELISA measurements for Rspondin 3 concentrations in EC supernatants from either LPS-activated or control ECs from three independent repeats; $n=6$ samples per group (mean \pm s.d.), two-sided unpaired Student's $t$-test was determined using GraphPad Prism, ${ }^{\star \star \star \star} P<0.0001$. d, Levels of anti-inflammatory markers (CD206, CD301, arginase 1 and IL-10) and pro-inflammatory markers (CD86, CD80, TNF and iNOS) as measured by flow cytometry in BMDMs stimulated with Rspondin3 $\left(40 \mathrm{ng} \mathrm{ml}^{-1}\right)$ or LPS $\left(100 \mathrm{ng} \mathrm{ml}^{-1}\right)$ alone or a combination of both for $24 \mathrm{~h}$ from three independent experiments with $n=3$ samples per group (mean \pm s.d.). Statistical significance was determined by two-way ANOVA with Tukey's multiple comparisons test using GraphPad Prism and individual $P$ values (left to right) are: CD206 ( $\left.{ }^{\star \star \star \star} P<0.0001,{ }^{\star \star \star \star \star} P<0.0001\right) ; C D 301\left({ }^{\star \star \star \star} P<0.0001,{ }^{\star \star \star \star} P<0.0001\right) ;$ arginase $\left.1{ }^{\star \star \star \star \star} P<0.0001,{ }^{\star \star \star \star \star} P<0.0001\right)$; IL-10 ( $\left.{ }^{\star \star \star \star} P<0.0001,{ }^{\star \star \star \star} P<0.0001\right) ; \mathrm{CD} 86$ (NS, $\left.P=0.1457,{ }^{\star \star \star \star} P<0.0001\right) ; \mathrm{CD} 80$ (NS, $\left.P=0.1576,{ }^{\star \star \star \star} P<0.0001\right) ;$ TNF $\left({ }^{\star \star \star \star \star} P<0.0001,{ }^{\star \star \star \star \star} P<0.0001\right) ;$ and iNOS $\left({ }^{\star \star * \star} P<0.0001,{ }^{\star \star * \star} P<0.0001\right)$. e, Heat map representing fold changes of anti-inflammatory cytokines and pro-inflammatory cytokines measured by ELISA in supernatants from BMDMs treated with Rspondin3 or LPS alone or a combination of both for $24 \mathrm{~h}$ from three independent experiments. TGF- $\beta$, transforming growth factor- $\beta$. f, Heat map representing gene expression levels (mean relative quantification (RQ) value) of anti-inflammatory marker genes (Mrc1, Arg1, Retnla, Chil3, Pparg and II10) and pro-inflammatory marker genes (Cd86, Cxcl1, II16, Tnf, II6 and Nos2) as measured by QPCR in BMDMs treated with Rspondin3 or LPS alone or a combination of both for $24 \mathrm{~h}$ from three independent experiments. 
(CD86, CD80, CD69, iNOS and TNF) were also analyzed by CyTOF (Fig. 2c and Extended Data Fig. 3b,c). We observed that endotoxemia in WT mice significantly increased the expression of pro-inflammatory markers at $24 \mathrm{~h}$ in IMs (Fig. $2 \mathrm{c}$ and Extended Data Fig. 3b) and that these inflammatory markers were reduced by $50 \%$ at $48 \mathrm{~h}$ post-LPS (although the values remained greater than the basal condition) (Fig. $2 c$ and Extended Data Fig. 3b). These pro-inflammatory markers in IMs were twofold to threefold greater in $R s p o 3^{\mathrm{EC}-1-}$ mice than those observed in WT mice at $24 \mathrm{~h}$ after LPS administration and were maintained at these high levels at $48 \mathrm{~h}$ (Fig. $2 \mathrm{c}$ and Extended Data Fig. 3b). We observed that i.v. Rspondin3 markedly reduced the expression of pro-inflammatory markers in IMs in both WT and Rspo $3^{\mathrm{EC}-1-}$ mice (Fig. 2c and Extended Data
Fig. 3b). The anti-inflammatory markers (CD206, CD301, RELM- $\alpha$, arginase 1 and IL-10) increased twofold to fourfold in IMs as compared to the basal condition in response to LPS for $24 \mathrm{~h}$ and continued to increase fourfold to sixteen fold in response to LPS at $48 \mathrm{~h}$ in WT mice (Fig. 2c and Extended Data Fig. 3b). These findings show that the activation of adaptive anti-inflammatory programming of IMs is elicited by endotoxemia. However, in $R s p o 3^{\mathrm{EC}-I-}$ mice, deletion of endothelial Rspo3 impaired the induction of these anti-inflammatory markers in IMs at $24 \mathrm{~h}$ after LPS challenge and this impairment became even more evident at $48 \mathrm{~h}$ post-LPS challenge (Fig. 2c and Extended Data Fig. 3b). Furthermore, we observed that the impairment of anti-inflammatory IM programming in $R s p o 3^{\mathrm{EC}-1-}$ mice was markedly restored by i.v. Rspondin 3

a
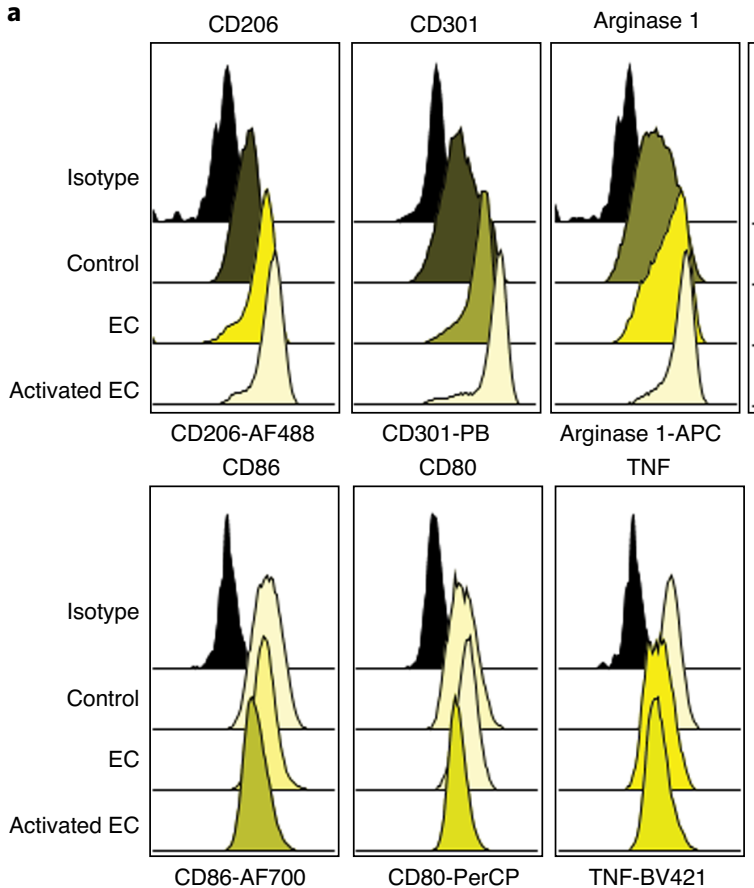

IL-10

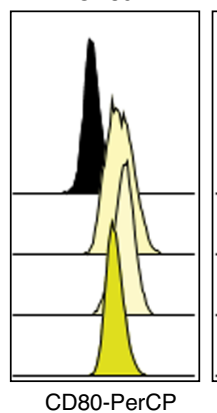

TNF
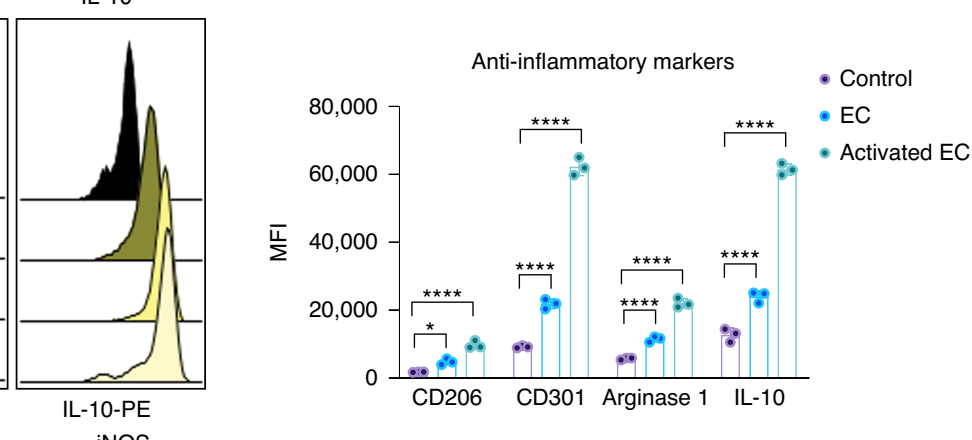

b
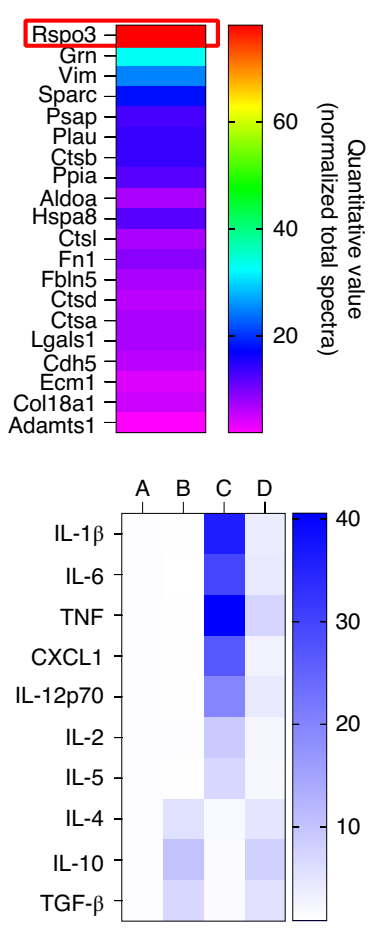

c
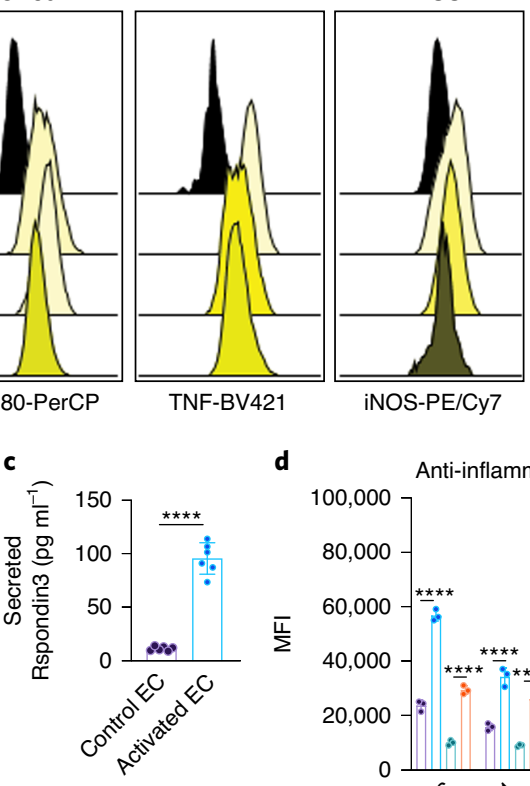

d
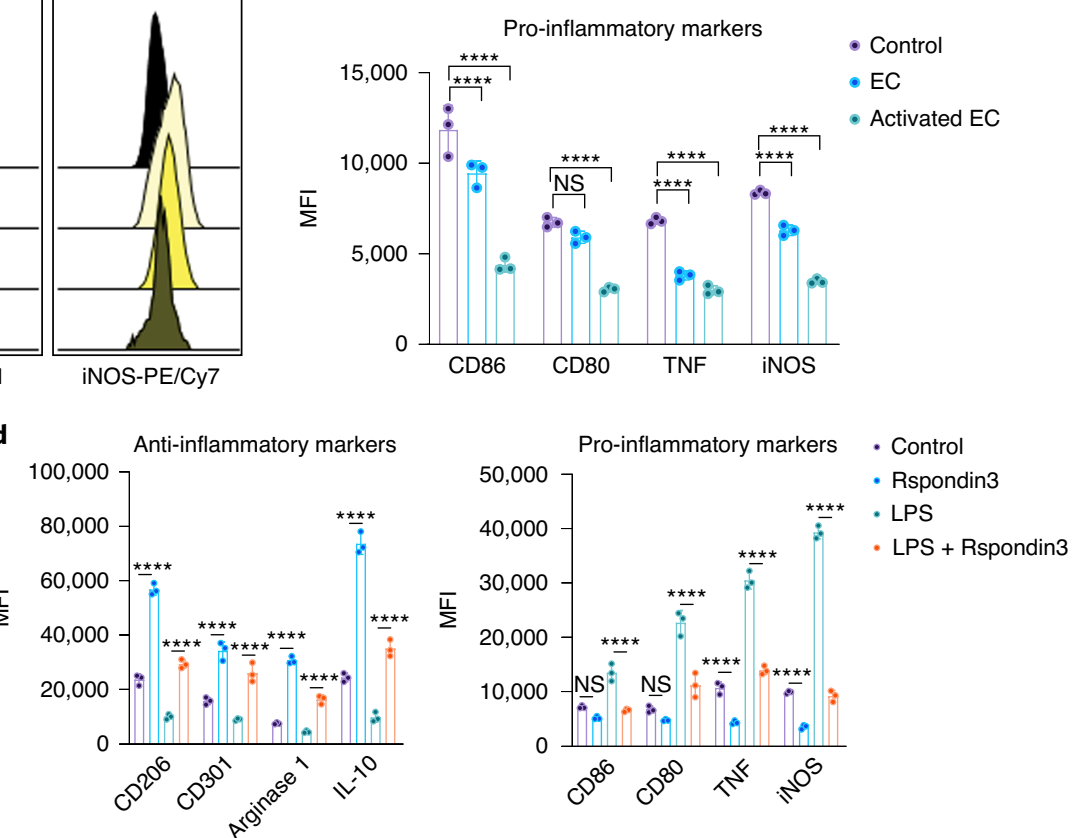

f

A: Control

B: Rspondin3

C: LPS

D: LPS + Rspondin3

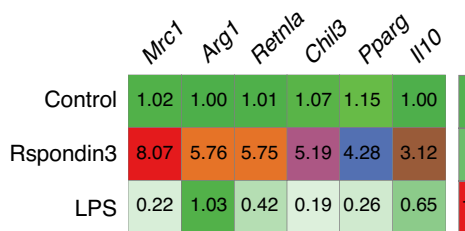

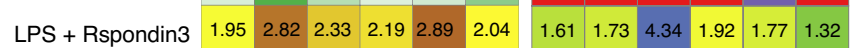

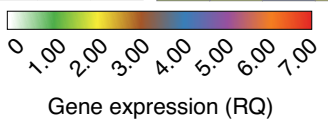


(Fig. $2 \mathrm{c}$ and Extended Data Fig. 3b). In contrast to what we observed in IMs, the expression of anti-inflammatory and pro-inflammatory markers in AMs did not differ between WT and Rspo $3^{\mathrm{EC}-1-}$ mice, as assessed by CyTOF (Extended Data Fig. 3c). These data suggested that angiocrine Rspondin3 specifically regulates IMs but not other lung myeloid cells in mice.

We next addressed whether the lung IM phenotype shift induced by endothelial Rspondin3 impacted the extent of inflammatory lung injury. Lung inflammation was assessed by quantifying neutrophil infiltration using the myeloperoxidase (MPO) activity assay as well as by quantifying changes in lung vascular permeability using the albumin-Evans blue dye tracer. Deletion of endothelial Rspo3 (Rspo ${ }^{\mathrm{EC}-1-}$ versus WT) had no effect on lung inflammation at baseline, whereas it markedly enhanced lung inflammatory injury during endotoxemia (Fig. 2d,e). Survival studies also showed that endothelial-specific deletion of Rspo3 significantly increased mortality as compared to control mice (Fig. 2f). The i.v. Rspondin3 acted as a therapeutic that attenuated lung inflammatory injury and enhanced survival in endotoxemic Rspo $3^{\mathrm{EC}-1-}$ mice (Fig. $2 \mathrm{~d}-\mathrm{f}$ ). These data demonstrated that Rspondin3-induced IM phenotypic transition prevents inflammatory lung injury.

We also used flow sorting to isolate AMs and IMs and performed gene profiling by quantitative PCR (qPCR). We observed that induction of the anti-inflammatory genes (Arg1, Chil3, Retnla, Mrc1, Clec10a, Il10, Clec7a, Pparg and Tgfb1) was significantly reduced in IMs obtained from endotoxemic Rspo $3^{\mathrm{EC}-/-}$ mice when compared to control mice (Extended Data Fig. 4a). In contrast, activation of pro-inflammatory genes (Cxcl1, Tnf, Nos2, Il1b, Cd80, $C d 86$ and $C d 69$ ) was markedly enhanced in IMs from endotoxemic Rspo $3^{\mathrm{EC}-1-}$ mice (Extended Data Fig. 4a). However, both the expression of anti-inflammatory and pro-inflammatory markers in AMs was not affected by the absence of endothelial Rspondin3 during the basal state or during endotoxemia (Extended Data Fig. 4a).

To establish whether the observed role of endothelial Rspondin3 was also applicable to other forms inflammatory lung injury, we used a bleomycin model, in which bleomycin is administered intratracheally to induce inflammatory $\mathrm{ALI}^{15}$. At day 5 post-bleomycin (ALI phase), WT and Rspo $3^{\mathrm{EC}-1-}$ mice were killed and myeloid cells were analyzed by CyTOF. We observed that among lung myeloid populations, monocytes, neutrophils and eosinophils were not affected by bleomycin-induced ALI; DC numbers increased after bleomycin but no difference was found between WT and Rspo $3^{\mathrm{EC}-l-}$ mice (Extended Data Fig. 4b). AMs decreased by $60 \%$ after bleomycin administration but there was no difference between WT and Rspo3 $3^{\mathrm{EC}-/-}$ mice (Extended Data Fig. 4b). IMs expanded threefold in WT mice after bleomycin exposure, whereas IMs in Rspo $3^{\mathrm{EC}-l-}$ mice were unable to mount this expansion response to injury (Extended Data Fig. 4b). The anti-inflammatory and pro-inflammatory phenotypes for lung macrophages following bleomycin-induced inflammatory lung injury were also analyzed by CyTOF (Extended Data Fig. 4c,d). Endothelial-specific deletion of Rspo3 shifted the lung IM population toward a pro-inflammatory phenotype in response to bleomycin-induced lung injury and thus mirrored the effects we had seen in endotoxemia-induced injury. These data suggested that the role of endothelial Rspondin3 as a regulator of an anti-inflammatory lung IM phenotype is a generalizable principle in lung inflammatory injury.

LGR4 is required for Rspondin3-induced macrophage transition. Rspondin family proteins induce Wnt- $\beta$-catenin signaling by binding to members of the leucine-rich repeat-containing $G$ protein receptor (LGR) family (Lgr4, Lgr5 and Lgr6) $)^{21-23}$. We found that only Lgr4 was highly expressed in macrophages (Supplementary Fig. 2a,b). Depletion of Lgr4 in macrophages abrogated Rspondin3-induced expression of the anti-inflammatory marker CD206 and reversed Rspondin3-induced suppression of the pro-inflammatory marker CD86 (Fig. 3a). We also observed that Lgr4 depletion dampened Rspondin3-induced gene expression of anti-inflammatory markers and promoted activation of pro-inflammatory genes in BMDMs in response to LPS (Fig. 3b and Extended Data Fig. 5a). Furthermore, depletion of Lgr4 augmented the release of pro-inflammatory cytokine IL- $1 \beta$ in BMDMs in response to LPS and prevented the release of anti-inflammatory cytokine IL-10 induced by Rspondin 3 (Fig. 3c). These together, indicated the requisite role of LGR4 in mediating Rspondin3-induced macrophage phenotype transition.

We found that Rspondin3 activated Wnt signaling in BMDMs in a LGR4-dependent manner (Fig. 3d). To investigate the role of LGR4 in lung macrophages in vivo, we first evaluated its expression in IMs, AMs and other myeloid cells by CyTOF and found that LGR4 was highly expressed in IMs as opposed to AMs and other

Fig. 2 | Rspondin3 mediates lung IM phenotype transition and prevents inflammatory lung injury. $\mathbf{a}$, Identifying lung macrophages as well as other myeloid cells by CyTOF. Resident macrophages were identified by CD45+F4/80+Ly6G-Ly6C-CD64+MerTK+ and IMs and AMs were further identified

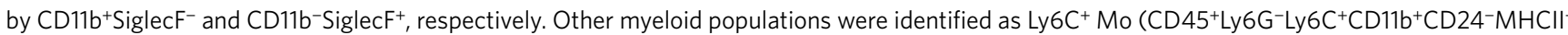
SiglecF-CD206-); Ly6C- Mo (CD45+Ly6G-Ly6C-CD11b+CD24-MHCII-SiglecF-CD206-); neutrophils (CD45+Ly6G+CD116+F4/80-); CD103+ DCs (CD

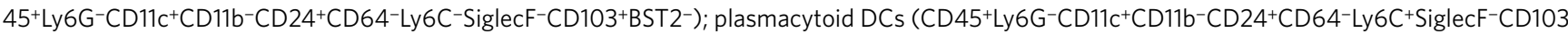
$\left.{ }^{-B S T 2}{ }^{+}\right) ; C^{2} 11 b^{+}$DCs (CD45+Ly6G-CD11C+CD11b+CD24+CD64-Ly6C-SiglecF-CD103-BST2--) and eosinophils (CD45+ Ly6G-Ly6C-CD11b+CD24+Siglec $\left.\mathrm{F}^{+} \mathrm{CD} 11 \mathrm{c}^{-} \mathrm{MHCII}^{-} \mathrm{CD}^{-} 4^{-}\right)$. b. Absolute cell number for lung IMs and AMs in WT and Rspo3 ${ }^{\mathrm{EC}-/-}$ mice with or without i.v. Rspondin3 ( $\left.0.25 \mathrm{mg} \mathrm{kg}{ }^{-1}\right)$ under basal conditions and following post-sublethal LPS challenge $\left(12 \mathrm{mg} \mathrm{kg}^{-1}\right.$, i.p.) for $24 \mathrm{~h}$ or $48 \mathrm{~h}$ as measured by CyTOF (data are representative of three independent experiments with five mice per group). Graphs show the mean \pm s.d. with each dot representing an individual mouse. Statistical significance was determined by two-way ANOVA with Tukey's multiple comparisons test using GraphPad Prism and individual $P$ values (left to right) are: IMs (NS, $P=0.3306$; NS, $P=0.5741$; NS, $P=0.2460$; ${ }^{\star \star \star \star} P<0.0001$; ${ }^{* \star \star \star} P<0.0001 ;{ }^{* \star \star \star} P<0.0001 ;{ }^{\star \star \star \star} P<0.0001 ;{ }^{* \star \star \star} P<0.0001$; $\left.{ }^{\star \star \star \star} P<0.0001\right)$; and AMs (NS, $P=0.8041 ; N S, P=0.9768 ; N S, P=0.9902 ; N S, P=0.9835 ; N S, P=0.7590 ; N S, P=0.9409 ; N S, P=0.9998 ; N S, P=0.9862 ; N S, P=0.9844)$. c, Heat map showing levels of anti-inflammatory markers (top) and pro-inflammatory markers (bottom) in lung IMs in WT and Rspo3EC-/- mice with or without i.v. Rspondin3 under basal and post-sublethal LPS challenge for $24 \mathrm{~h}$ or $48 \mathrm{~h}$ as measured by CyTOF ( $n=5$ mice per group with three independent repeats, shown as fold changes by the mean CyTOF signal intensity normalized to control group). d, Lung vascular permeability was measured by using the albumin-Evans blue dye tracer (EBA) in WT and Rspo3 ${ }^{\mathrm{EC}-1-}$ mice with or without i.v. Rspondin3 under basal and post-sublethal LPS challenge for $24 \mathrm{~h}$ or $48 \mathrm{~h}$ (data are representative of three independent experiments with five mice per group). Graphs show mean \pm s.d. with each dot representing an individual mouse. Statistical significance was determined by two-way ANOVA with Tukey's multiple comparisons test using GraphPad Prism and individual $P$ values (left to right) are: NS, $P=0.9997 ; \mathrm{NS}, P=0.9996$; ${ }^{\star \star \star \star} P<0.0001 ;{ }^{\star \star \star \star} P<0.0001 ;{ }^{\star \star \star \star \star} P<0.0001 ;{ }^{\star \star \star \star \star} P<0.0001 ;{ }^{\star \star \star \star} P<0.0001 ;{ }^{* \star \star \star} P<0.0001$. e, MPO activity of flushed lung samples from WT and Rspo3 ${ }^{\mathrm{EC}-/-}$ mice with or without i.v. Rspondin3 under basal and post-sublethal LPS challenge for $24 \mathrm{~h}$ or $48 \mathrm{~h}$ (data are representative of three independent experiments with five mice per group). Graphs show mean \pm s.d. with each dot representing an individual mouse. Statistical significance was determined by two-way ANOVA with Tukey's multiple comparisons test using GraphPad Prism and individual $P$ values (left to right) are: NS, $P=0.9774$; NS, $P=0.9715$; ${ }^{\star \star \star \star} P<0.0001$; ${ }^{\star \star \star \star} P<0.0001$; ${ }^{\star \star \star \star} P<0.0001$; ${ }^{\star \star \star \star} P<0.0001 ;{ }^{\star} P=0.0250 ;{ }^{\star \star \star \star \star} P<0.0001$. f, Survival curves for WT and Rspo3 ${ }^{\mathrm{EC}-/-}$ mice with or without i.v. Rspondin3 during endotoxemia conditions $(n=16$ mice for each group). 
myeloid populations (Extended Data Fig. 5b,c and Supplementary Fig. 2e). In vivo, with macrophage-specific Lgr4 knockout mice (herein called $L g r 4^{\mathrm{M} \varphi-/-}$ ) (Supplementary Fig. 2f), we found that L $g r 4^{\mathrm{M} \varphi-l-}$ mice showed exacerbated inflammatory lung injury responses as compared to WT mice induced by sublethal LPS challenge, as measured by lung MPO activity and albumin-Evans blue dye tracer assessment of lung vascular permeability (Fig. 3h,i). These changes could not be rescued by i.v. Rspondin 3 as was the case in WT mice (Fig. 3h,i). The number of IMs in $\operatorname{Lgr} 4^{\mathrm{M} \varphi-/-}$ mice also failed to increase in response to endotoxemia by i.v. Rspondin3 (Fig. 3e). Furthermore, expression of anti-inflammatory markers and pro-inflammatory markers in $\operatorname{Lgr} 4^{\mathrm{M} \varphi-/-}$ mice and control mice during endotoxemia was analyzed by CyTOF. We found that deletion of Lgr4 prevented the generation of IMs and failed to induce expression of anti-inflammatory markers, which was coupled with marked increases in pro-inflammatory markers in response to

a
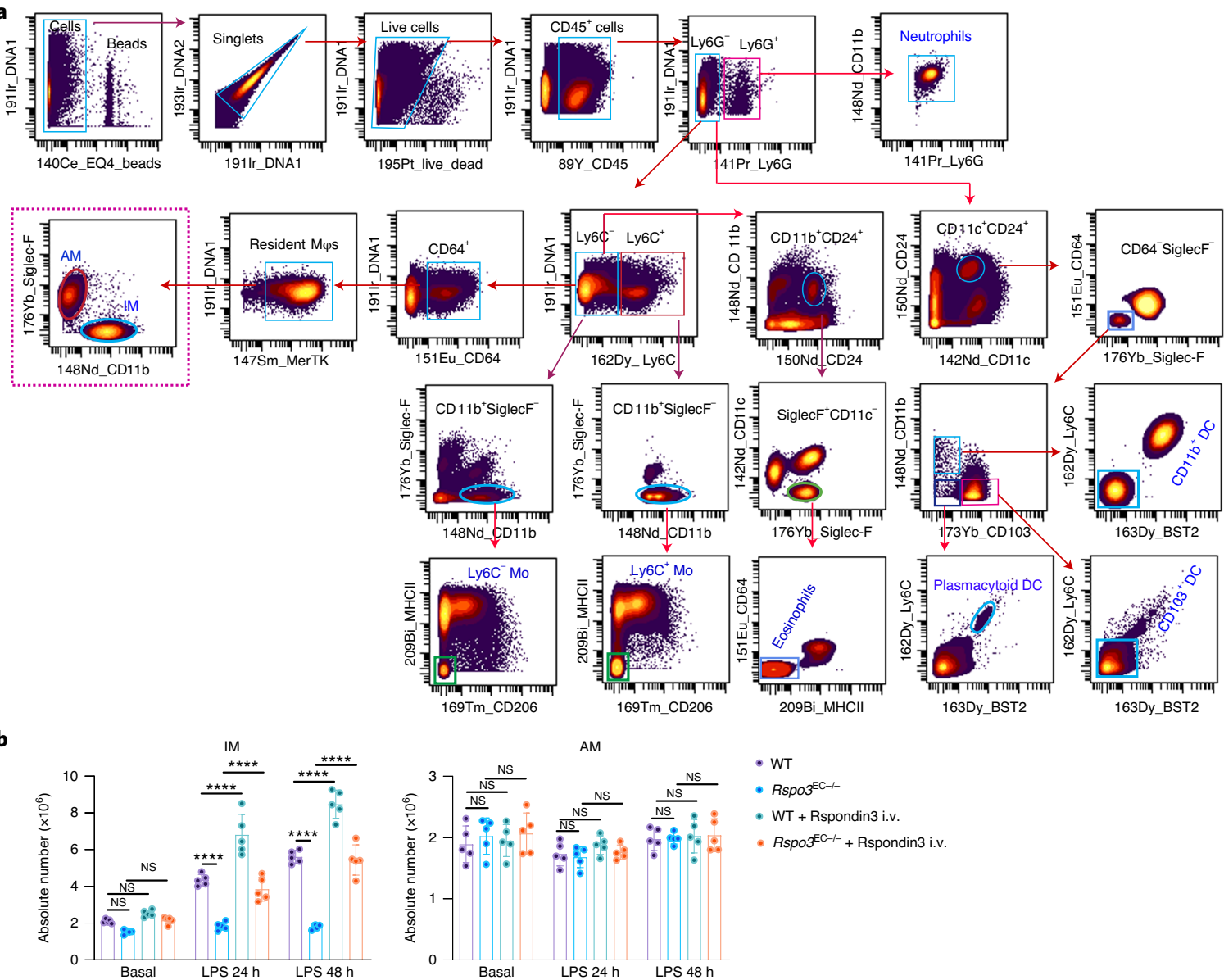

WT

- Rspo3 ${ }^{\mathrm{EC}-1-}$

- $\mathrm{WT}+\mathrm{Rspondin} 3$ i.v.

- Rspo3 ${ }^{\mathrm{EC}-1-}+$ Rspondin3 i.v.

C

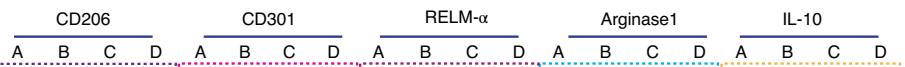

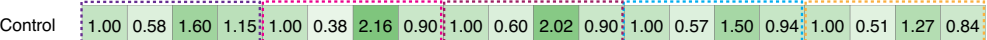

\begin{tabular}{l|lllll|l|l|l|l|l|l|l|l|l|l|l|l|l|l|l} 
LPS $24 \mathrm{~h}$ & 3.47 & 1.06 & 8.57 & 2.35 & 3.94 & 1.28 & 5.99 & 2.86 & 1.91 & 0.91 & 4.41 & 1.54 & 2.06 & 1.06 & 3.79 & 1.71 & 1.57 & 0.83 & 2.12 & 1.44
\end{tabular}

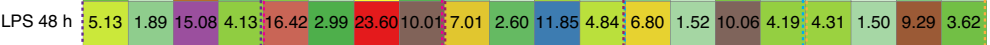

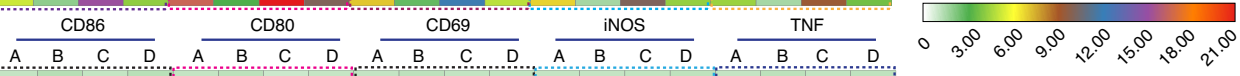

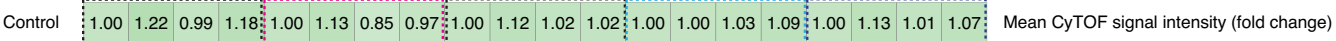

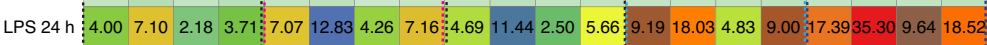

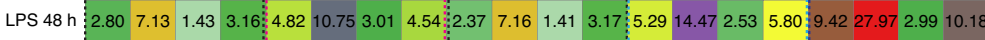

d

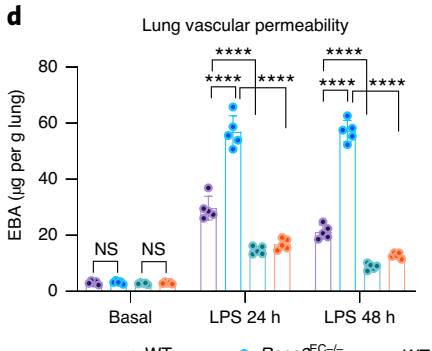

- WT e

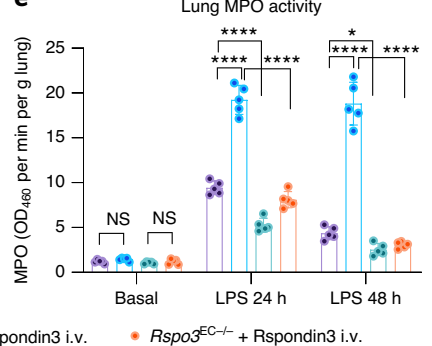

B: Rspo3 ${ }^{\mathrm{EC}-1}$

C: WT + Rspondin3 i.v.

Rspo3 ${ }^{\mathrm{EC}-/-}+$ Rspondin3 i.v.

f

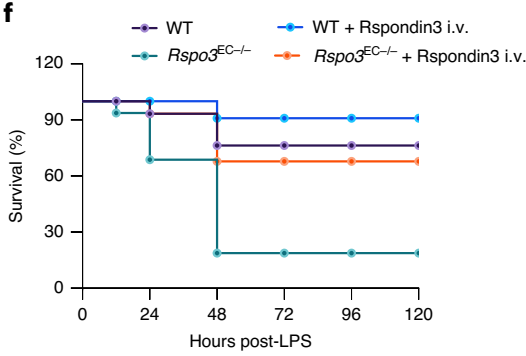


endotoxemia; furthermore, these changes were not reversed by i.v. Rspondin3 (Fig. 3e,f). Also, in Lgr $4^{\mathrm{M} \varphi-l-}$ mice, Rspondin3 failed to induce Wnt- $\beta$-catenin signaling in IMs as determined by measuring active $\beta$-catenin (nonphosphorylated $\beta$-catenin) (Fig. $3 g$ ). Taken together, these data showed LGR4 is the receptor in IMs required for Rspondin3-mediated reprograming of macrophages and Rspondin3-mediated attenuation of inflammatory lung injury.

及-catenin signals Rspondin3-induced macrophage transition. Rspondin family proteins activate both $\beta$-catenin-dependent canonical Wnt- $\beta$-catenin signaling and $\beta$-catenin-independent noncanonical Wnt signaling ${ }^{22,24}$. We next determined whether $\beta$-catenin was essential for Rspondin3-mediated generation of IMs and prevention of inflammatory lung injury. Mice with a genetic deletion of Ctnnb1 (encoding $\beta$-catenin) in macrophages $\left(\right.$ Lyz2-cre ${ }^{+}$Ctnnb1 ${ }^{\mathrm{fl} / \mathrm{fl}}$, herein called $C$ tnnb $^{\mathrm{M} \varphi-/-}$ ) and $C \mathrm{Ctnnb}^{\mathrm{f} / / \mathrm{fl}}$ (WT) were generated (Supplementary Data Fig. 3a). We measured Ctnnb1 expression in BMDMs prepared from Ctnnb $1^{\mathrm{M} \varphi-/-}$ mice and WT mice (Supplementary Data Fig. 3b,c) and measured active $\beta$-catenin in IMs in vivo by CyTOF (Supplementary Data Fig. 3d). We observed that deletion of Ctnnb1 in macrophages abrogated Rspondin3-induced expression of the anti-inflammatory marker CD206 and instead increased expression of the pro-inflammatory marker CD86 (Fig. 4a). We also found that deletion of Ctnnb1 in macrophages suppressed expression of the anti-inflammatory genes induced by Rspondin 3 and promoted induction of pro-inflammatory marker genes in
BMDMs in response to LPS challenge (Fig. 4a and Extended Data Fig. 6). ELISA confirmed that deletion of Ctnnb1 augmented the release of the pro-inflammatory cytokine IL-1 $\beta$ in BMDMs in response to LPS and prevented the release of anti-inflammatory cytokine IL-10 by Rspondin3 (Fig. 4c). In vivo, the number of IMs in Ctnnb1 $1^{\mathrm{M} \varphi-/-}$ mice failed to increase in response to endotoxemia, and this failure could not be rescued by i.v. Rspondin 3 as it was in the WT mice (Fig. 4d). Furthermore, Ctnnb1 $1^{\mathrm{M} \varphi-l-}$ mice showed a failure to induce expression of anti-inflammatory markers in lung IMs, whereas it concomitantly increased the expression of pro-inflammatory markers in response to endotoxemia; this could not be reversed by i.v. Rspondin3 (Fig. 4e). We found that Ctnnb1 $1^{\mathrm{M} \varphi-/-}$ mice demonstrated augmented inflammatory lung injury and mortality in response to endotoxemia, which could not be rescued by i.v. Rspondin 3 (Fig. $4 \mathrm{f}-\mathrm{h}$ ). Thus, these results showed that macrophage $\beta$-catenin was required for Rspondin3-induced IM phenotype transition and Rspondin3-mediated attenuation of inflammatory lung injury.

Rspondin3 reprograms metabolism in macrophages. To address whether the Rspondin3-induced macrophage phenotype transition involved a specific metabolic reprogramming, we carried out a metabolic flux assay to detect changes in the mitochondrial oxygen consumption rate (OCR) and rate of extracellular acidification (ECAR), as measures of OXPHOS and glycolysis, respectively. In BMDMs cultured in full DMEM containing $10 \mathrm{mM}$ glucose, $2 \mathrm{mM}$

Fig. 3 | LGR4 expressed in macrophages is required for Rspondin3-induced macrophage phenotype transition. a, Representative flow cytometry overlay histogram and quantified data for the levels of anti-inflammatory marker CD206 and pro-inflammatory marker CD86 in BMDMs with or without (siNC, nontarget control short interfering RNA) Lgr4 depletion at basal condition or treated with Rspondin3, LPS alone or a combination of both for $24 \mathrm{~h}$ from three independent experiments with $n=3$ samples per group (mean \pm s.d.). Statistical significance was determined by two-way ANOVA Tukey's multiple comparisons using GraphPad Prism. $P$ values (left to right) are: CD206 ( $\left.{ }^{\star \star \star \star} P<0.0001 ;{ }^{\star \star \star \star} P<0.0001 ; N S, P=0.9520 ; N S, P=0.8256\right)$ and $C D 86$ ${ }^{\star} P=0.0286 ;{ }^{\star \star \star \star} P<0.0001 ;$ NS, $P=0.9252 ;$ NS, $P=0.0619$ ). b. Heat map representing the expression levels (mean RQ value) of anti-inflammatory marker genes (Mrc1, Arg1, Retnla and Chil3) and pro-inflammatory marker genes ( $C d 86, C x c 11, \| 17 b$ and Tnf) measured by qPCR in BMDMs with or without Lgr4 depletion treated with Rspondin3 or LPS alone or a combination of both for $24 \mathrm{~h}$ from three independent experiments. $\mathbf{c}$, Concentrations of the anti-inflammatory cytokine IL-10 and pro-inflammatory cytokine IL-1 $\beta$ in supernatants of BMDMs with or without Lgr4 depletion measured by ELISA from three independent experiments with $n=3$ samples per group (mean \pm s.d.). Statistical significance was determined by two-way ANOVA with Sidak's multiple comparisons using GraphPad Prism. $P$ values (left to right) are: IL-10 (NS, $P=0.9995 ;{ }^{\star \star \star \star} P<0.0001 ; N S, P=0.9643 ;{ }^{* \star \star \star} P<0.0001$ ) and IL-1 $\beta$ (NS, $P=0.9908 ; N S, P=0.1029 ;{ }^{\star \star \star \star} P<0.0001$; $\left.{ }^{\star \star \star \star} P<0.0001\right)$. d, Wnt signaling activity in BMDMs as measured by TOP-Flash reporter assays from three repeats with three samples per group (mean \pm s.d.). Statistical significance was determined by two-way ANOVA with Sidak's multiple comparisons using GraphPad Prism. P values (left to right) are: ${ }^{\star \star \star \star} P<0.0001 ;$ NS, $P>0.9999$. e, Absolute cell numbers of IMs in WT and Lgr4M ${ }^{M}-/-$ mice with or without i.v. Rspondin3 under basal and after sublethal LPS challenge for $24 \mathrm{~h}$ measured by CyTOF (data are representative of three independent experiments with five mice per group). Graphs show mean \pm s.d. with each dot representing an individual mouse. Statistical significance was determined by two-way ANOVA with Tukey's multiple comparisons test using GraphPad Prism and individual $P$ values (left to right) are: NS, $P=0.5877 ;$ NS, $P=0.3916 ; \mathrm{NS}, P=0.3806 ;{ }^{\star \star} P=0.0055 ;{ }^{\star \star \star \star} P<0.0001 ; \mathrm{NS},=0.9983$. f, Levels of anti-inflammatory and pro-inflammatory markers in IMs in WT and $\operatorname{Lgr} 4^{\mathrm{M} \varphi-/-}$ mice with or without i.v. Rspondin3 under basal and post-sublethal LPS challenge for $24 \mathrm{~h}$ measured by CyTOF (data are representative of three independent experiments with five mice per group). Graphs show mean \pm s.d. with each dot representing an individual mouse. Statistical significance was determined by two-way ANOVA with Tukey's multiple comparisons test using GraphPad Prism and individual $P$ values (left to right) are: CD206 (NS, $\left.P=0.1062 ; N S, P=0.7861 ; N S, P=0.9854 ;{ }^{* \star \star \star} P<0.0001 ;{ }^{\star \star \star \star} P<0.0001 ; N S, P=0.9719\right) ; C D 301$ (NS, $P=0.9222 ; N S, P=0.7557 ; N S$, $P=0.9865 ;{ }^{\star \star} P=0.0027$; $\left.{ }^{\star \star \star \star} P<0.0001 ; N S, P=0.9810\right)$; arginase 1 (NS, $P=0.1902 ; \mathrm{NS}, P=0.5609 ; \mathrm{NS} P=0.9958$; ${ }^{\star \star \star \star} P<0.0001 ;{ }^{\star \star \star \star} P<0.0001 ; \mathrm{NS}$, $P=0.9608$ ); IL-10 (NS, $P=0.1421 ; N S, P=0.6669 ;$ NS, $P=0.9775 ; * \star \star \star P<0.0001 ; * \star * \star P<0.0001 ; N S P=0.8189) ; C D 86$ (NS, $P=0.2238 ; N S, P>0.9999$; NS, $\left.P>0.9999 ;{ }^{\star \star \star \star} P<0.0001 ;{ }^{\star \star \star} P=0.0009 ; \mathrm{NS}, P=0.5211\right) ; \mathrm{CD} 80$ (NS, $P=0.3158 ; \mathrm{NS}, P=0.9885 ; \mathrm{NS} P=0.9998 ;{ }^{* \star \star \star} P<0.0001 ;{ }^{\star \star} P=0.0062 ; \mathrm{NS}$, $P=0.9579$ ); iNOS (NS, $P=0.2172 ; \mathrm{NS}, P=0.9996 ; \mathrm{NS}, P=0.9930 ;{ }^{\star \star \star} P<0.0001 ; * \star P=0.0020 ; \mathrm{NS}, P=0.9150$ ); TNF (NS, $P=0.2758 ; \mathrm{NS}, P=0.9947$; NS, $P>0.9999 ;{ }^{* \star \star \star} P<0.0001$; ${ }^{\star \star \star \star} P<0.0001$; NS, $P=0.9986$ ). g, Active $\beta$-catenin (nonphospho) levels in IMs in WT and $L g r 4{ }^{M} \varphi-/-$ mice with or without i.v. Rspondin3 under basal and post-sublethal LPS challenge for $24 \mathrm{~h}$ as measured by CyTOF (data are representative of three independent experiments with five mice per group). Graphs show the mean \pm s.d. with each dot representing an individual mouse. Statistical significance was determined by two-way ANOVA with Tukey's multiple comparisons test using GraphPad Prism and individual $P$ values (left to right) are: NS, $P=0.1588$; ${ }^{\star \star \star \star} P<0.0001$; NS, $P>0.9999 ;{ }^{* \star \star} P=0.0001 ;{ }^{\star \star \star \star} P<0.0001 ; \mathrm{NS}, P=0.9971$. h, Lung vascular permeability was measured using EBA dye tracer in WT and $L g r 4 \mathrm{M} \varphi-/-$ mice with or without i.v. Rspondin3 under basal conditions and post-sublethal LPS challenge for $24 \mathrm{~h}$ (data are representative of three independent experiments with five mice per group). Graphs show mean \pm s.d. with each dot representing an individual mouse. Statistical significance was determined by two-way ANOVA with Tukey's multiple comparisons test using GraphPad Prism and individual $P$ values (left to right) are: NS, $P>0.9910$; ${ }^{* \star \star \star} P<0.0001$; ${ }^{\star * \star \star} P<0.0001 ; \mathrm{NS}, P=0.8521$. i, MPO activity of flushed lung samples from WT and L $\mathrm{gr}^{\mathrm{M} \varphi \mathrm{p}-/-}$ mice with or without i.v. Rspondin3 under basal and post-sublethal LPS challenge for $24 \mathrm{~h}$ (data are representative of three independent experiments with five mice per group). Graphs show mean \pm s.d. with each dot representing an individual mouse. Statistical significance was determined by two-way ANOVA with Tukey's multiple comparisons test using GraphPad Prism and individual $P$ values (left to right) are: NS, $P>0.9990 ;{ }^{\star \star \star \star} P<0.0001 ;{ }^{\star \star \star \star} P<0.0001 ; N S, P=0.5800$. 
glutamine and $2 \mathrm{mM}$ sodium pyruvate, we observed that Rspondin 3 markedly increased mitochondrial respiration as measured by the basal OCR and the spare respiratory capacity (SRC), which is assessed by mitochondrial uncoupling (Fig. $5 \mathrm{a}-\mathrm{c}$ ). At the same time, Rspondin 3 treatment reduced basal ECAR (Extended Data Fig. 7a). We also found that Rspondin3 treatment of LPS-activated BMDMs was able to reverse the metabolic phenotype of pro-inflammatory macrophages by preventing upregulation of glycolysis and by restoring mitochondrial oxygen consumption (Fig. 5a-c and Extended Data Fig. 7a). To determine the precise carbon source utilized by Rspondin3-increased mitochondrial respiration, we used DMEM containing only glucose, glutamine or free fatty acids as substrates for the metabolic assay, respectively. Here we observed that Rspondin 3 failed to increase mitochondrial OCR in macrophages
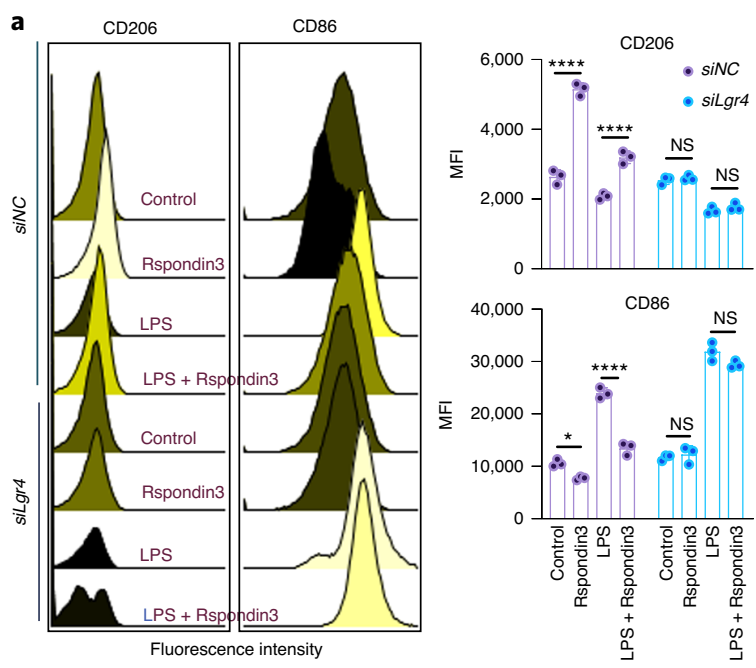

b
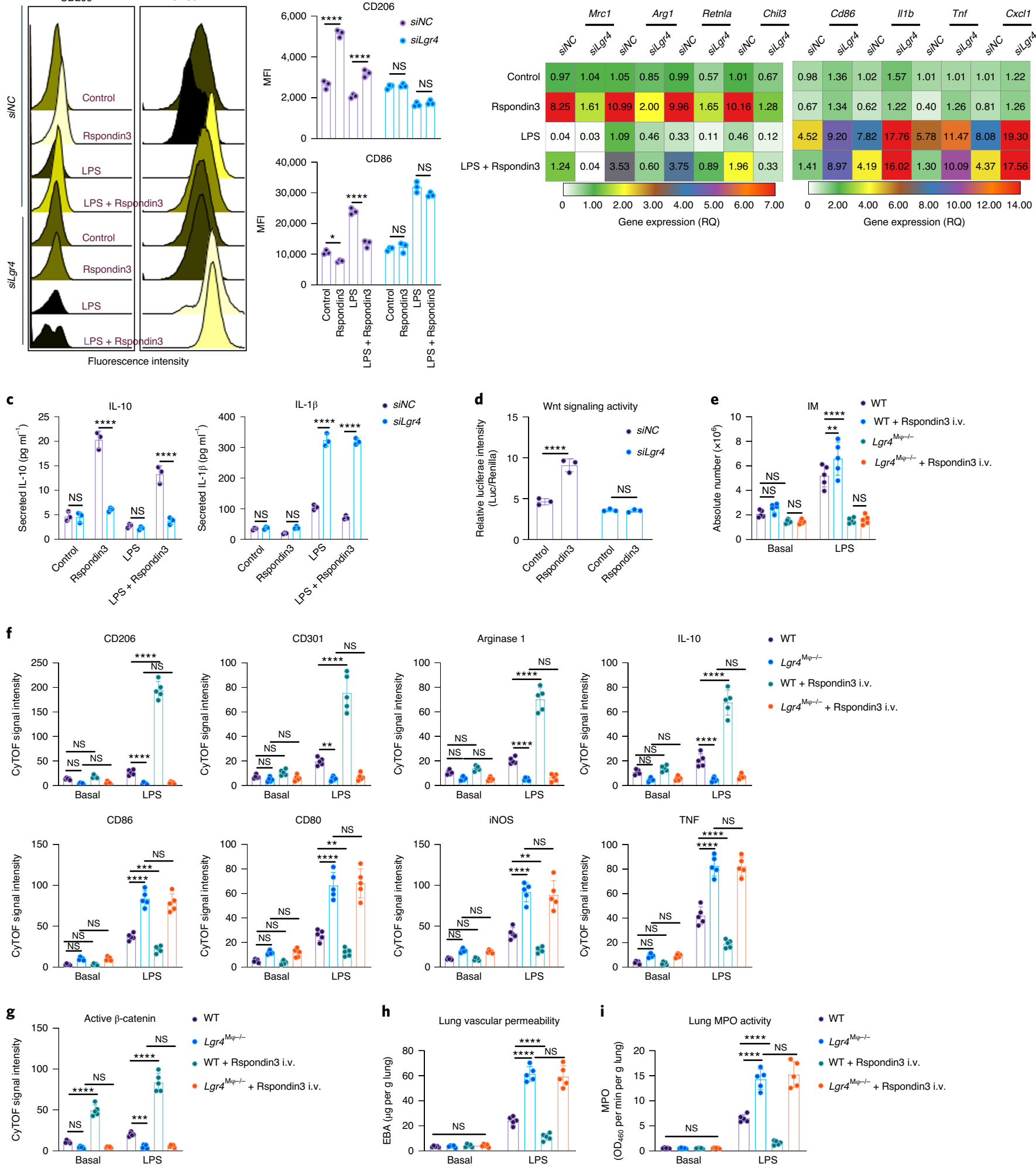
exposed to either glucose or free fatty acids as substrates (Fig. 5g-1), whereas Rspondin3 markedly increased OCR with glutamine as the sole substrate (Fig. $5 \mathrm{~d}-\mathrm{f}$ ). We also observed that Rspondin3 increased the activity of glutaminase, which is responsible for glutaminolysis (Extended Data Fig. 7f) and induced gene expression of glutaminase $(G l s)$ (Extended Data Fig. $7 \mathrm{~g}$ ). Thus, these results showed that the Rspondin3-induced anti-inflammatory switch in macrophages involved increased OXPHOS via glutaminolysis. Next, using macrophages prepared from $\operatorname{Lgr} 4^{\mathrm{M} \varphi-/-}$ mice and $\operatorname{Ctnnb} 1^{\mathrm{M} \varphi-/-}$ mice, we observed that deletion of Lgr4 or Ctnnb1 abrogated Rspondin3-induced changes in OCR and ECAR (Fig. 5m-O and Extended Data Fig. 7e). Rspondin3-mediated increases in the activity of glutaminase and levels of Gls were prevented in macrophages from $\operatorname{Lgr}^{\mathrm{M} \varphi-/-}$ mice and Ctnnb $1^{\mathrm{M} \varphi-/-}$ mice (Extended Data Fig. 7f,g). Thus, Rspondin3-mediated reprogramming of macrophages required the activation of glutaminolysis via LGR $4 / \beta$-catenin signaling.

Rspondin3 induces epigenetic reprogramming through $\alpha$-ketoglutarate-TET2 to activate anti-inflammatory transition. Glutaminolysis induces the generation of $\alpha$-ketoglutarate, which serves as the cofactor for DNA hydroxymethylation catalyzed by TET methylcytosine dioxygenase or histone demethylation catalyzed by JMJD3 demethylase ${ }^{25-27}$. We thus addressed whether the Rspondin3-mediated increase of $\alpha$-ketoglutarate generation (Fig. 6a) induced epigenetic reprogramming in DNA hydroxymethylation or histone methylation. Measurements of TET and JMJD3 activities using nuclear extracts from macrophages with or without Rspondin3 stimulation showed that Rspondin3 increased TET activities (Fig. 6b), whereas there was no effect on JMJD3 activities (Supplementary Fig. 4a). As the TET enzymes induce DNA hydroxymethylation and open chromatin and activate gene expression in the associated loci ${ }^{28}$, we determined whether Rspondin 3 induced DNA hydroxymethylation in macrophages. Global 5-hydroxymethylcytosine $(5 \mathrm{hmC})$ measurements showed that Rspondin 3 markedly increased $5 \mathrm{hmC}$ levels and prevented LPS-induced $5 \mathrm{hmC}$ downregulation in macrophages (Fig. 6c). Furthermore, 5hmC DNA immunoprecipitation followed by qPCR (hMeDIP-qPCR) used to detect $5 \mathrm{hmC}$ enrichment on specific gene loci showed notable enrichment of $5 \mathrm{hmC}$ within the proximal promoter regions of anti-inflammatory genes (Mrc1, Arg1, Chil3 and Retnla) (Fig. 6d and Extended Data Fig. 8a). Chromatin immunoprecipitation (ChIP) with an antibody specific for $\mathrm{H} 3 \mathrm{~K} 4 \mathrm{me} 3$, the active histone marker for open chromatin regions and transcription active genes, followed with qPCR (ChIP-qPCR) targeting anti-inflammatory gene promoters (Mrc1, Arg1, Chil3 and Retnla) also showed that Rspondin3 activated the chromatin state on these gene loci (Fig. 6e and Extended Data Fig. 8b). However, in macrophages obtained from $\operatorname{Lgr} 4^{\mathrm{M} \varphi-l-}$ mice or Ctnnb1 $1^{\mathrm{M} \varphi-/-}$ mice, in which Lgr4 or Ctnnb1 was deleted in macrophages, we observed that Rspondin 3 failed to increase $\alpha$-ketoglutarate levels, TET activity and $5 \mathrm{hmC}$ levels as well as activation of anti-inflammatory genes (Fig. 6a-e and Extended Data Fig. 8a,b). Thus, the Rspondin3-induced metabolic shift enhanced $\alpha$-ketoglutarate generation in macrophages, which activated the epigenetic program to catalyze DNA hydroxymethylation by TETs that utilize $\alpha$-ketoglutarate as a cofactor.

Using macrophage Tet 2 knockout mice $\left(L y z 2-\mathrm{cre}^{+} ; \mathrm{Tet} 2^{\mathrm{f} / \mathrm{fl}}\right.$, herein called Tet $2^{\mathrm{M} \varphi-l-}$ ) (Supplementary Fig. $4 \mathrm{c}-\mathrm{e}$ ), we observed that the number of IM in Tet $2^{\mathrm{M} \varphi-/-}$ mice failed to increase in response to endotoxemia and they could not be rescued by i.v. Rspondin3 (Extended Data Fig. 8d). Tet $2^{\mathrm{M} \varphi-/-}$ mice also showed a failure of IMs

Fig. 4 | $\beta$-catenin signals Rspondin3-induced macrophage phenotype transition. a, Representative flow cytometry overlay histograms and quantified data for the levels of the anti-inflammatory marker CD206 and pro-inflammatory marker CD86 shown by MFI in BMDMs from WT and Ctnnb7 ${ }^{\mathrm{M} \varphi-/-}$ mice at the baseline condition or treated with Rspondin3 or LPS alone or a combination of both for $24 \mathrm{~h}$ from three independent experiments with $n=3$ samples per group (mean \pm s.d.). Statistical significance was determined by two-way ANOVA with Tukey's multiple comparisons using GraphPad Prism. $P$ values (left to right) are: CD206 ( $\left.{ }^{\star \star \star \star} P<0.0001 ;{ }^{\star \star} P=0.0029 ; N S, P=0.9982 ; N S, P=0.9765\right)$ and $C D 86\left({ }^{\star} P=0.0318 ;{ }^{* \star \star \star} P<0.0001 ; N S, P=0.8020 ; N S\right.$, $P=0.1195)$. $\mathbf{b}$, Heat map representing gene expression levels (shown as mean RQ value) of anti-inflammatory marker genes (Mrc1, Arg1, Retnla and Chil3) and pro-inflammatory marker genes ( $C d 86, C x C l 1,1 / 1 b$ and $T n f$ ) measured by QPCR in BMDMs from WT and Ctnnb $7^{\mathrm{M} \varphi-/-}$ mice in the baseline condition or treated with Rspondin 3 or LPS alone or a combination of both for $24 \mathrm{~h}$ from three independent repeats. c, Concentrations of the anti-inflammatory cytokine IL-10 and pro-inflammatory cytokine IL-1 $\beta$ in supernatants measured by ELISA in BMDMs from WT and Ctnnb $7^{\mathrm{M} \varphi-/-}$ mice at the baseline condition or treated with Rspondin3, LPS alone or a combination of both for $24 \mathrm{~h}$ from three independent experiments with $n=3$ samples per group (mean \pm s.d.). Statistical significance was determined by two-way ANOVA with Sidak's multiple comparisons using GraphPad Prism. $P$ values (left to right) are: IL-10 (NS, $P=0.9742 ;{ }^{\star \star \star \star} P<0.0001$; NS, $\left.P=0.7458 ;{ }^{\star \star \star \star} P<0.0001\right)$ and IL-1 $\beta$ (NS, $P=0.9995 ;$ NS, $\left.P=0.9936 ;{ }^{\star \star \star \star} P<0.0001 ;{ }^{\star \star \star \star} P<0.0001\right)$. d, Absolute cell number for IMs in WT and Ctnnb $7^{\mathrm{M} \varphi-/-}$ mice with or without i.v. Rspondin3 under basal state and post-sublethal LPS challenge for $24 \mathrm{~h}$ measured by CyTOF (data are representative of three independent experiments with five mice per group). Graphs show mean \pm s.d. with each dot representing an individual mouse. Statistical significance was determined by two-way ANOVA with Tukey's multiple comparisons test using GraphPad Prism and individual $P$ values (left to right) are: NS, $P=0.6725 ;{ }^{\star *} P=0.0069 ; \mathrm{NS}, P=0.9991 ;{ }^{\star \star \star \star} P<0.0001 ;{ }^{\star \star \star \star} P<0.0001 ; \mathrm{NS}, P=0.9595$. e, Levels of anti-inflammatory markers (CD206, CD301, arginase 1 and IL-10) and pro-inflammatory markers (CD86, CD80, iNOS and TNF) in lung IMs in WT and Ctnnb7 ${ }^{\mathrm{M} \varphi-/-}$ mice with or without i.v. Rspondin3 under basal and post-sublethal LPS challenge for $24 \mathrm{~h}$ as measured by CyTOF (data are representative of three independent experiments with five mice per group). Graphs show mean \pm s.d. with each dot representing an individual mouse. Statistical significance was determined by two-way ANOVA with Tukey's multiple comparisons test using GraphPad Prism and individual $P$ values (left to right) are: CD206 (NS, $P=0.2099$; NS, $P=0.7433 ;$ NS, $P=0.9536$; ${ }^{\star \star \star \star} P<0.0001$; ${ }^{\star \star \star \star} P<0.0001 ; N S, P=0.9178$ ); CD301 (NS, $P=0.8729 ; N S, P=0.9144 ; N S, P=0.9998 ;{ }^{\star \star \star \star \star} P<0.0001$; ${ }^{\star \star \star \star} P<0.0001 ; \mathrm{NS}, P=0.8828$ ); arginase 1 (NS, $P=0.3580 ; \mathrm{NS}, P=0.9857 ; \mathrm{NS}, P=0.6133$; ${ }^{\star \star \star \star} P<0.0001 ;{ }^{\star \star \star \star} P<0.0001 ; \mathrm{NS}, P=0.8287$ ); IL-10 (NS, $\left.P=0.1331 ; N S, P=0.2284 ; N S, P=0.6216 ;{ }^{\star \star \star \star} P<0.0001 ;{ }^{* \star \star \star} P<0.0001 ; N S, P=0.1970\right) ; C D 86$ (NS, $P=0.9977 ; N S, P=0.7760 ; N S, P=0.9908$; $\left.{ }^{\star \star \star \star} P<0.0001 ;{ }^{\star \star \star} P=0.0008 ; N S, P=0.9614\right) ; C D 80$ (NS, $P=0.6493 ; N S, P=0.8283 ; N S, P=0.8780 ;{ }^{* \star \star \star} P<0.0001 ;{ }^{* \star \star} P=0.0008 ; N S, P=0.7463$ ); iNOS (NS, $P=0.8586 ;$ NS, $P=0.9763 ;$ NS, $P=0.9937$; ${ }^{\star \star \star \star} P<0.0001 ;{ }^{\star \star \star} P=0.0004 ;$ NS, $\left.P=0.9994\right) ;$ TNF (NS, $P=0.9677 ; N S, P=0.8926 ;$ NS, $P=0.9740 ;{ }^{\star \star \star \star} P<0.0001 ;{ }^{\star \star \star \star} P<0.0001$; NS, $\left.P=0.7855\right)$. f, Lung vascular permeability as measured in WT and Ctnnb $7^{\mathrm{M} \varphi-/-}$ mice with or without i.v. Rspondin3 under basal and post-sublethal LPS challenge for $24 \mathrm{~h}$ (data are representative of three independent experiments with five mice per group). Graphs show mean \pm s.d. with each dot representing an individual mouse. Statistical significance was determined by two-way ANOVA with Tukey's multiple comparisons test using GraphPad Prism and individual $P$ values (left to right) are: NS, $P=0.9997 ; \mathrm{NS}, P=0.9973 ; \mathrm{NS}, P=0.9998$; ${ }^{\star \star \star \star} P<0.0001$; ${ }^{\star} P=0.0254 ; \mathrm{NS}, P=0.9603$. g, MPO activity of flushed lung samples from WT and Ctnnb $7^{\mathrm{M} \varphi-/-}$ mice with or without i.v. Rspondin3 under basal and post-sublethal LPS challenge for $24 \mathrm{~h}$ (data are representative of three independent experiments with five mice per group). Graphs show mean \pm s.d. with each dot representing an individual mouse. Statistical significance was determined by two-way ANOVA with Tukey's multiple comparisons test using GraphPad Prism and individual $P$ values (left to right) are: NS, $P=0.9996 ;$ NS, $P=0.9997 ; \mathrm{NS}, P>0.9999$; ${ }^{* \star \star \star *} P<0.0001 ;{ }^{\star \star \star \star} P<0.0001 ; \mathrm{NS}, P=0.9195$. h, Survival curve for WT and Ctnnb $7^{\mathrm{M} \varphi-/-}$ mice with or without i.v. Rspondin3 following sublethal LPS challenge ( $n=16$ mice for each group). 
to induce expression of anti-inflammatory markers, while markedly increasing expression of pro-inflammatory markers in response to endotoxemia, which could not be rescued by i.v. Rspondin3 (Fig. $6 f$ and Extended Data Fig. 8c). Tet2 deletion in macrophages exacerbated inflammatory lung injury induced by endotoxemia, which was not rescued by i.v. Rspondin3 (Fig. 6g,h). We observed that Tet 2 deletion prevented Rspondin3-induced DNA hydroxymethylation in macrophages (Fig. 6c-e and Extended Data Fig. 8a,b). We also observed $\beta$-catenin translocated to the nucleus of macrophages and markedly increased nuclear TET $2 / \beta$-catenin interaction as revealed by proximity ligation assays (Extended Data Fig. 6e,f). Therefore, in addition to the role of $\alpha$-ketoglutarate serving as the cofactor for TET2, direct nuclear $\beta$-catenin/TET2 interaction may contribute to epigenetic reprogramming. These data demonstrated that Rspondin3 epigenetically reprogrammed macrophages through TET2-catalyzed DNA hydroxymethylation as a consequence of
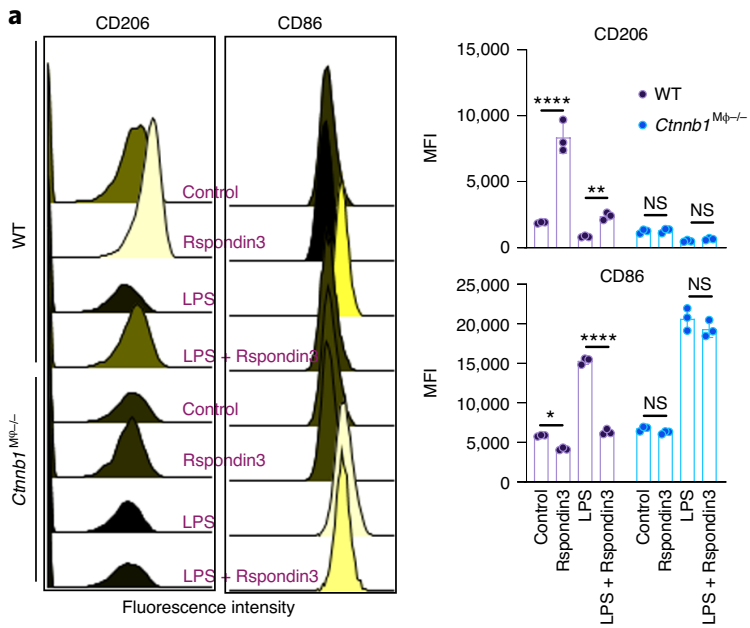

b
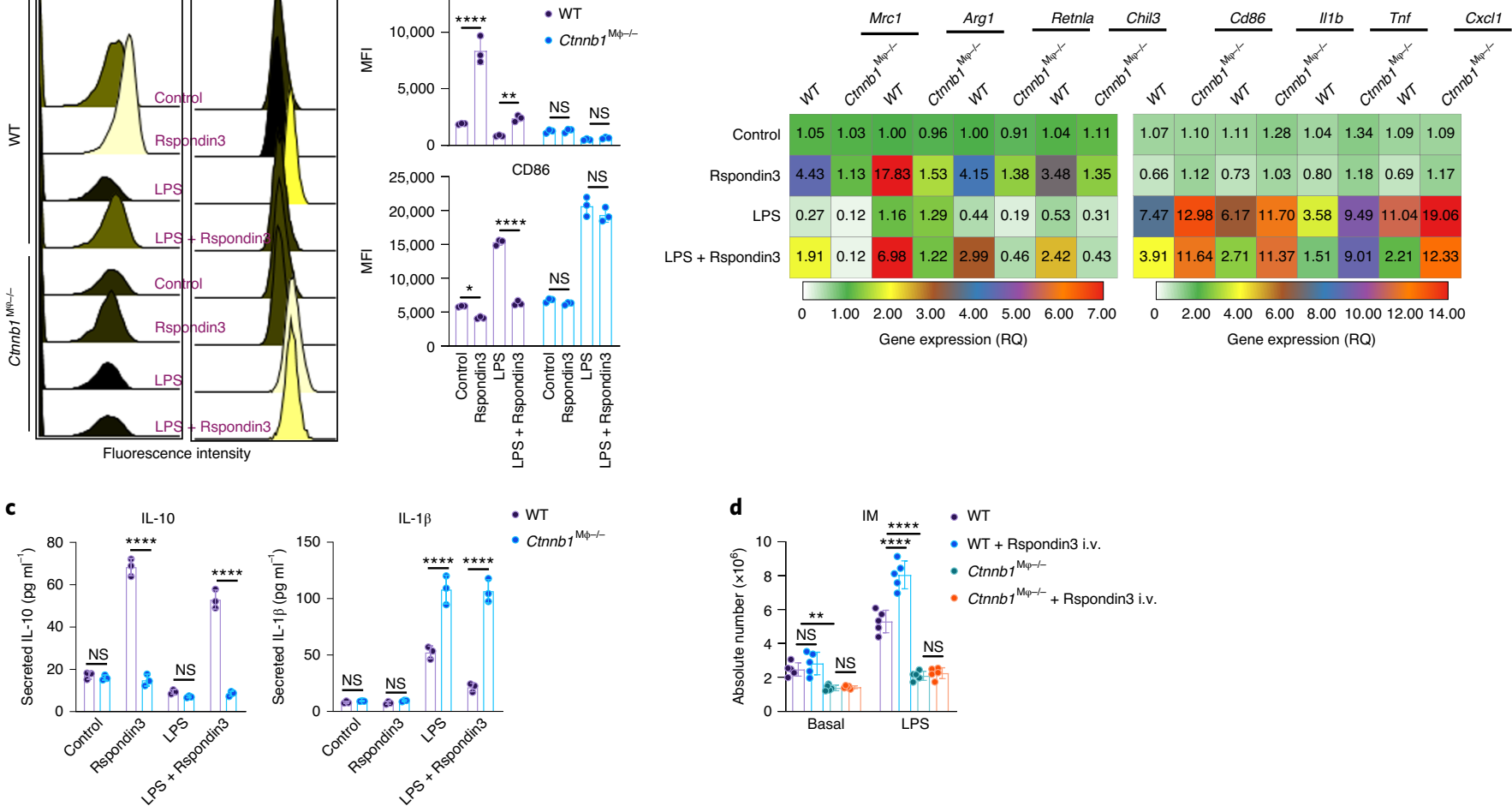

e
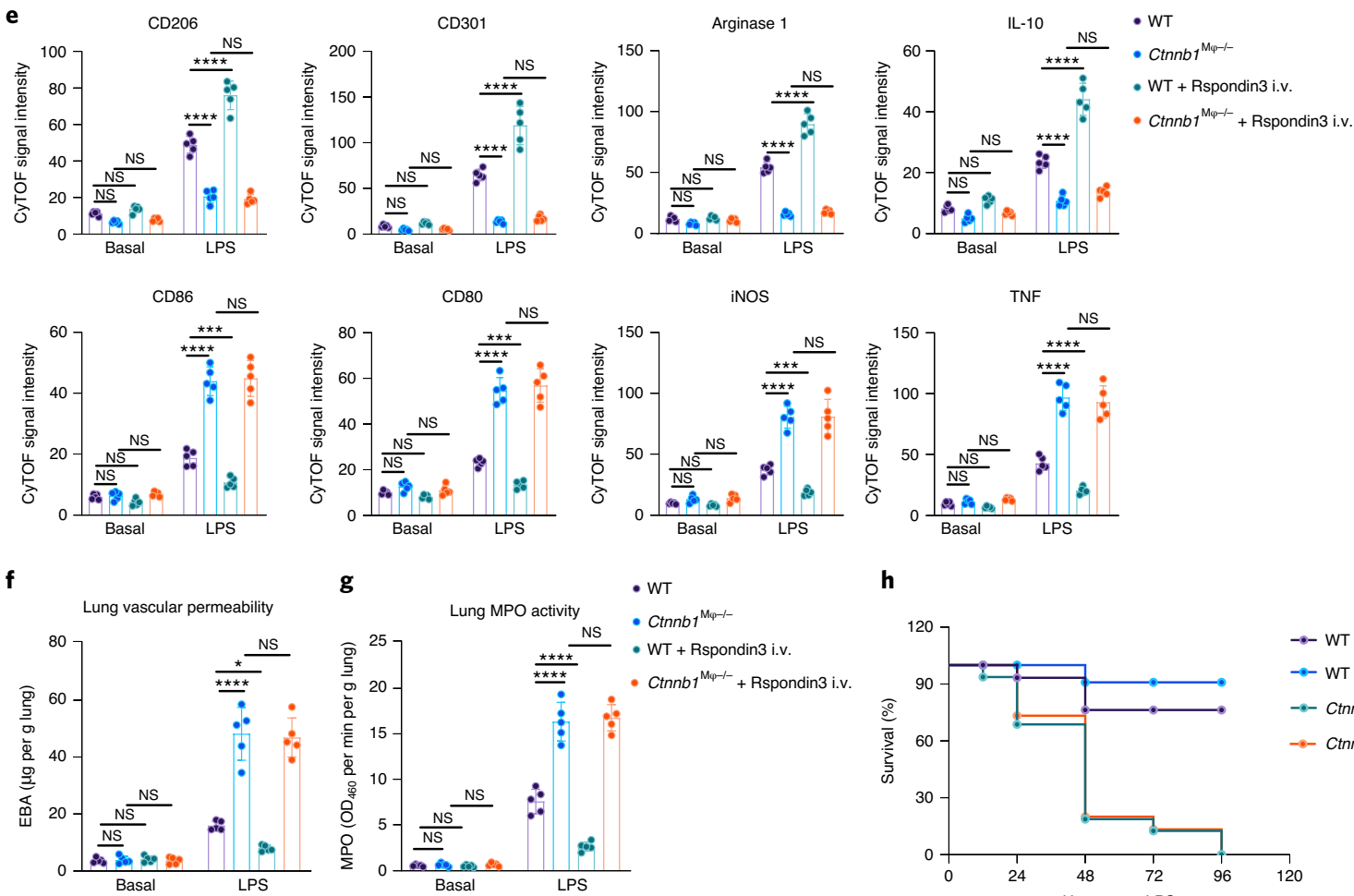

h

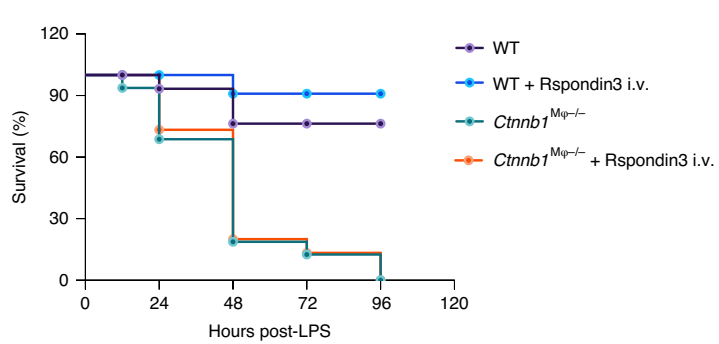


enhanced glutamine metabolism, which provided $\alpha$-ketoglutarate as the necessary cofactor mediating macrophage phenotype transition.

Taken together, these results show that TET2, the primary enzyme catalyzing DNA hydroxymethylation with $\alpha$-ketoglutarate as cofactor, was essential for reprogramming macrophages and thereby prevented inflammatory lung injury through the activation of Rspondin3-induced metabolic-epigenetic programs in macrophages (Extended Data Fig. 8g).

\section{Discussion}

The tissue niche is emerging as a crucial regulator of the identity, phenotype and function of resident macrophages ${ }^{10-12,29}$. Recent studies have identified metabolic shifts, epigenetic modifications and activation of specific transcriptional programs as key mediators of macrophage reprogramming and phenotype switch ${ }^{1,7,8,25,30,31}$. Here, we demonstrated that ECs are essential in driving the generation of a population of lung IMs with potent tissue-reparative and anti-inflammatory functions during inflammatory injury. The endothelium releases the Wnt signaling activator Rspondin 3 that acts as a key angiocrine signal, mediating the reprogramming of IMs. This transition is the result of metabolic-epigenetic reprogramming fueled by the activation of glutamine metabolism and generation of the metabolite $\alpha$-ketoglutarate, a requisite cofactor in TET2-mediated DNA hydroxymethylation ${ }^{32}$. Thus, Rspondin3 generated by ECs orchestrates both metabolic and epigenetic programs to promote lung IM transition toward an anti-inflammatory phenotype and thus promote resolution of inflammation and tissue repair as adaptive mechanisms during inflammatory injury.

The unique lung microenvironment has been demonstrated to be crucial for resident AM development, differentiation and plasticity $^{12,13,33,34}$, whereas little is known about the properties and generation of the population of IMs. Single-cell RNA sequencing recently identified subpopulations of $\mathrm{IMs}^{15,35}$. However, how the lung microenvironment imprints the phenotype and function of these cells remains unclear. In the present study, using CyTOF, which enabled high-dimensional analysis of macrophage phenotypes and rigorous analysis of IMs, AMs and other lung myeloid populations, we found a large absolute number of IMs that was comparable to AMs. These results are consistent with a recent study using design-based stereology showing a relatively large number of IMs in human lungs ${ }^{36}$. We also found that lung IM numbers further increased and reached levels significantly greater than AMs post-LPS challenge. This finding is consistent with studies demonstrating high turnover of lung IMs at steady-state and a profound capacity to expand in response to stressors ${ }^{16,37,38}$. Thus, our results are fully congruent with evidence that IMs represent an important lung resident macrophage population.

We determined the role of lung ECs in providing the niche signals that tailor lung IM phenotype and function, as IMs are located in close proximity to the lung vascular endothelial niche. We observed that Rspondin 3 was constitutively generated by ECs, whereas its release increased up to tenfold in response to LPS. We demonstrated that endothelial-derived Rspondin 3 bound to its cognate receptor LGR4 in macrophages and activated $\beta$-catenin-dependent Wnt signaling. Endothelial-specific genetic deletion of Rspo3 demonstrated that it was required for the maintenance of the lung IM population during homeostasis and for the expansion of IMs following experimental inflammatory injury induced by either endotoxemia or bleomycin in mice. The adaptive expansion and phenotypic switch toward anti-inflammatory

Fig. 5 | Rspondin3 increases mitochondrial respiration through glutamine metabolism. a, Mitochondrial OCR measured in BMDMs stimulated with Rspondin3, LPS alone or a combination of both for $24 \mathrm{~h}$ with complete DMEM containing $10 \mathrm{mM}$ glucose, $2 \mathrm{mM}$ glutamine and $2 \mathrm{mM}$ sodium pyruvate as substrate. Data are representative of three independent experiments, $n=10-12$ samples per group and graphs show the mean \pm s.d. $\mathbf{b}$, Bar figure for basal OCR in $\mathbf{a}$. Data are representative of three independent experiments, with 10-12 samples per group. Graphs show the mean \pm s.d., with each dot representing an individual sample per time point. Statistical significance was determined by ordinary one-way ANOVA with Tukey's multiple comparisons test using GraphPad Prism and $P$ values are: ${ }^{\star \star \star \star} P<0.0001$; ${ }^{\star \star \star} P=0.0003$. c, Bar figure for SRC in $\mathbf{a}$. Data are representative of three independent experiments with 10-12 samples per group. Graphs show mean \pm s.d. with each dot representing an individual sample per time point. Statistical significance was determined by ordinary one-way ANOVA with Tukey's multiple comparisons test using GraphPad Prism and $P$ values are: ${ }^{\star \star \star \star} P<0.0001$; $\star \star \star \star P<0.0001$. d, OCR measured in BMDMs stimulated with Rspondin3, LPS alone or a combination of both for $24 \mathrm{~h}$ with only $2 \mathrm{mM}$ glutamine as substrate. Data are representative of three independent experiments, $n=10-12$ samples per group and graphs show mean \pm s.d. e, Bar figure for basal OCR from d. Data are representative of three independent experiments, with 10-12 samples per group. Graphs show mean \pm s.d. with each dot representing an individual sample per time point. Statistical significance was determined by ordinary one-way ANOVA with Tukey's multiple comparisons test using GraphPad Prism and $P$ values are: ${ }^{\star \star \star \star} P<0.0001 ;{ }^{* \star \star *} P<0.0001$. f, Bar figure for SRC in $\mathbf{d}$. Data are representative of three independent experiments with 10-12 samples per group. Graphs show mean \pm s.d. with each dot representing an individual sample per time point. Statistical significance was determined by ordinary one-way ANOVA with Tukey's multiple comparisons test using GraphPad Prism and $P$ values are ${ }^{\star \star \star \star} P<0.0001 ;{ }^{* \star \star \star} P<0.0001$. g, OCR measured in BMDMs stimulated with Rspondin3, LPS alone or a combination of both for $24 \mathrm{~h}$ with only $10 \mathrm{nM}$ free fatty acid as palmitate bound to BSA added as substrate. Data are representative of three independent experiments, $n=10-12$ samples per group and graphs show the mean \pm s.d. h, Bar figure for basal OCR from $\mathbf{g}$. Data are representative of three independent experiments, with 10-12 samples per group. Graphs show mean \pm s.d. with each dot representing an individual sample well per time point. Statistical significance was determined by ordinary one-way ANOVA with Tukey's multiple comparisons test using GraphPad Prism and $P$ values are: NS, $P=0.10 ;$ NS, $P=0.0528$. i, Bar figure for SRC in $\mathbf{g}$. Data are representative of three independent experiments with 10-12 samples per group. Graphs show mean \pm s.d. with each dot representing an individual sample per time point. Statistical significance was determined by ordinary one-way ANOVA with Tukey's multiple comparisons test using GraphPad Prism and $P$ values are: NS, $P=0.2595 ; \mathrm{NS}, P=0.3785$. j, OCR measured in BMDMs stimulated with Rspondin3, LPS alone or a combination of both with only $10 \mathrm{mM}$ glucose as substrate. Data are representative of three independent experiments, $n=10-12$ samples per group and graphs show mean $\pm s$.d. $\mathbf{k}$, Bar figure for basal OCR in $\mathbf{j}$. Data are representative of three independent experiments with 10-12 samples per group. Graphs show mean \pm s.d. with each dot representing an individual sample per time point. Statistical significance was determined by ordinary one-way ANOVA with Tukey's multiple comparisons test using GraphPad Prism and P values are: NS, $P=0.9992 ; N S, P=0.9773$. I, Bar figure for SRC in $\mathbf{j}$. Data are representative of three independent experiments with 10-12 samples per group. Graphs show mean \pm s.d. with each dot representing an individual sample per time point. Statistical significance was determined by ordinary one-way ANOVA with Tukey's multiple comparisons test using GraphPad Prism and $P$ values are: NS, $P=0.9556 ; N S, P=0.8668$. $\mathbf{m}, O C R$ measurements in WT, $L g r 4^{\mathrm{M} \varphi-/-}$ and Ctnnb $7^{\mathrm{M} \varphi-/-}$ BMDMs stimulated with or without Rspondin3 in the medium with only $2 \mathrm{mM}$ glutamine as substrate. Data are representative of three independent experiments, $n=10-12$ samples per group and graphs show mean \pm s.d. $\mathbf{n}, \mathbf{o}$, Bar figure for basal OCR ( $\mathbf{n}$ ) and SRC (o) for $\mathbf{m}$. Data are representative of three independent experiments, with 10-12 samples per group. Graphs show mean \pm s.d. with each dot representing an individual well per time point. Statistical significance was determined by ordinary one-way ANOVA with Tukey's multiple comparisons test using GraphPad Prism and $P$ values are: ${ }^{\star \star \star \star} P<0.0001 ; N S, P>0.9999 ;$ NS, $P=0.4223$; ${ }^{\star \star \star \star} P<0.0001 ; N S, P>0.9999, N S, P=0.5107$. 
IMs during endotoxemia was essential for resolution of inflammation. This response was inactivated in the absence of angiocrine Rspondin3. The defective anti-inflammatory switch increased mortality from $15 \%$ in control mice to $80 \%$ in $R s p o 3^{\mathrm{EC}-/-}$ mice. Thus, Rspondin 3 mediated the phenotypic and functional regulation of IMs and was required for resolving inflammatory lung injury and reducing mortality. It has been recently reported that deletion of Rspondin receptor Lgr4 in myeloid cells enhanced the efficacy of checkpoint blockade therapy by switching the macrophage phenotype in tumors toward a pro-inflammatory state $^{39}$, and Lgr4-deficient mice were highly susceptible to LPSinduced septic shock due to the increased production of proinflammatory cytokines by macrophages ${ }^{40}$. Our findings complement these reports by demonstrating that LGR4 is the receptor mediating the anti-inflammatory and reparative effects of endothelial Rspondin3 on the macrophage phenotype.
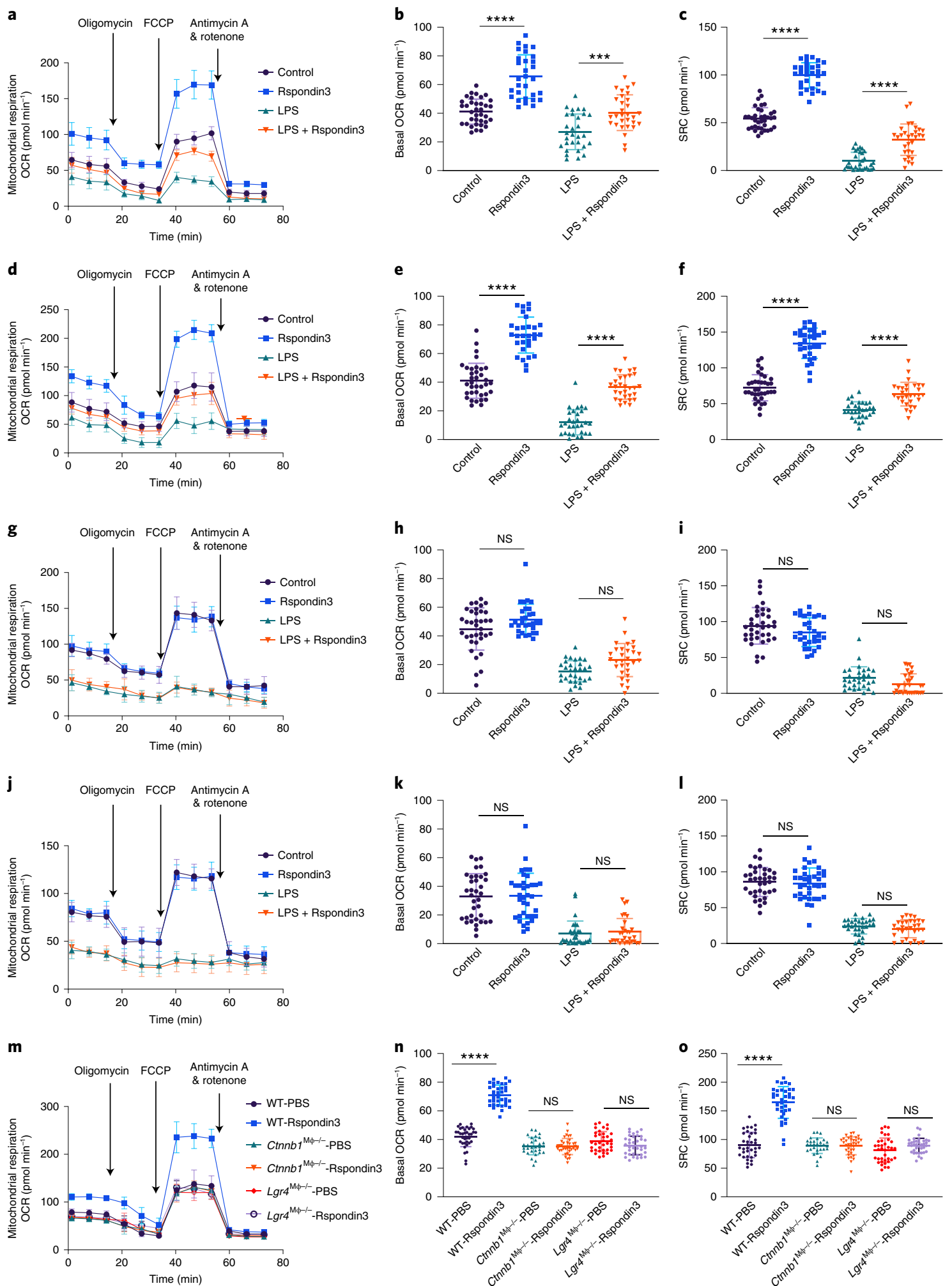


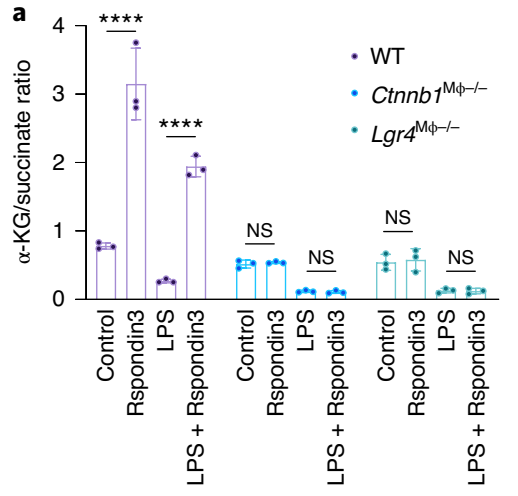

d

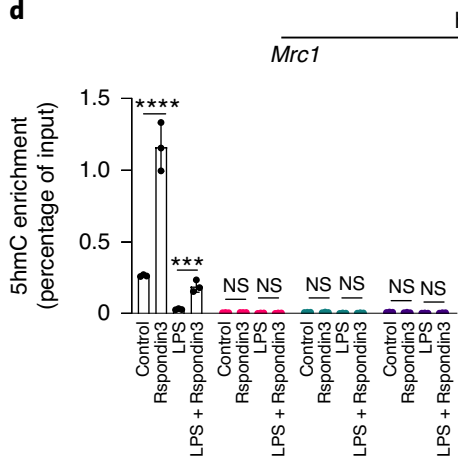

hMeDIP

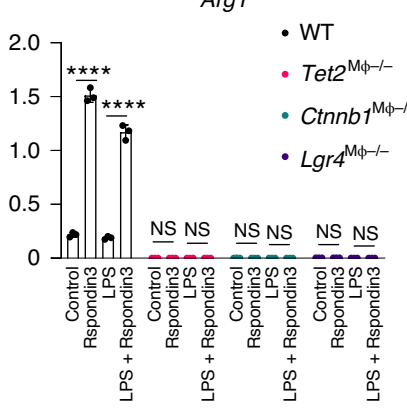

f

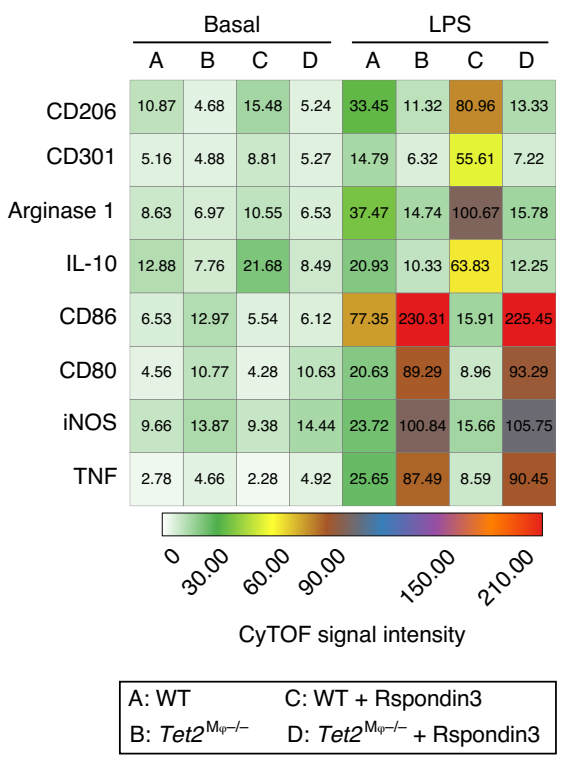

g

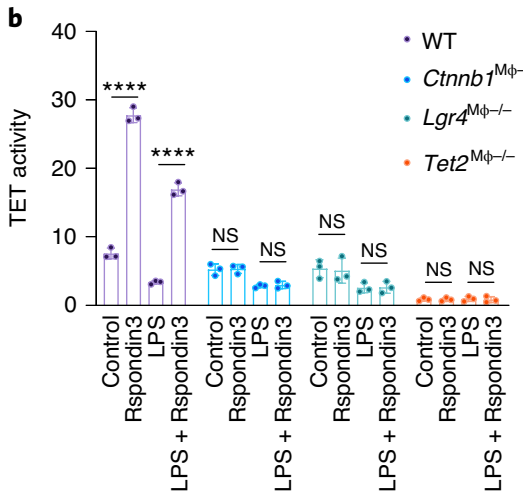

e

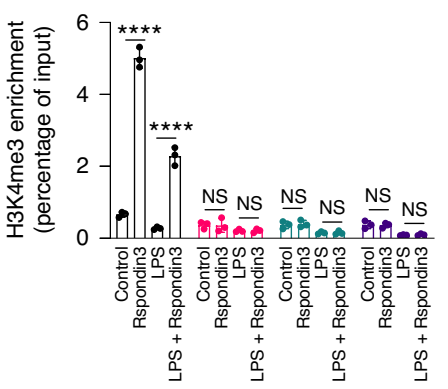

h
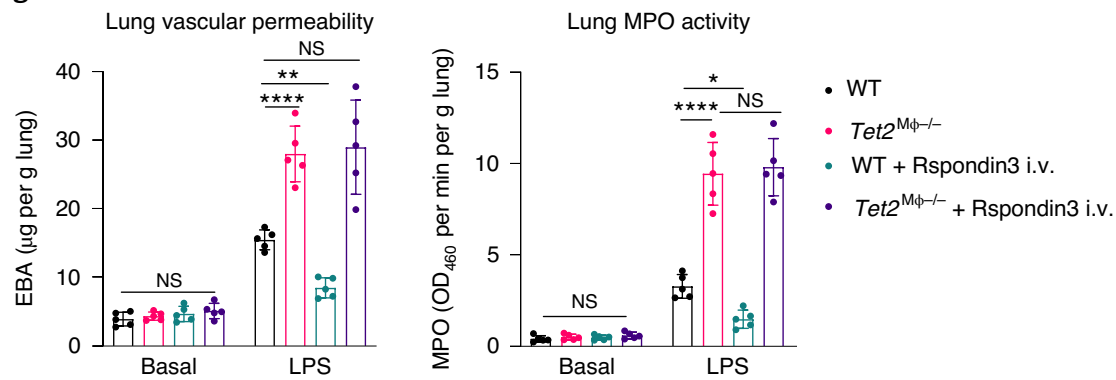

WT

- Ctnnb ${ }^{\mathrm{MQ} \phi-1-}$

- $\operatorname{Lgr} 4^{\mathrm{M} \phi-1-}$

- $T e t 2^{\mathrm{M} \phi-1-}$

E $0.04-\left.\right|^{\frac{* \star *}{2}}$

ֻे

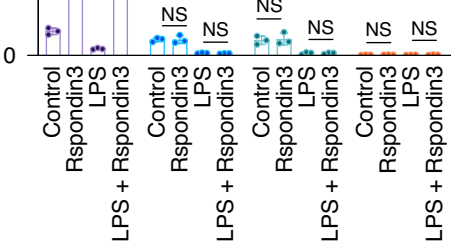

ChIP witn anti-H3K4me3

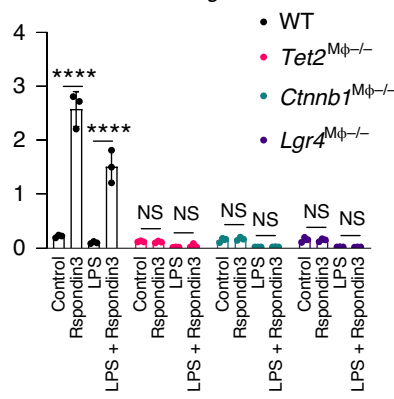


Fig. 6 | Rspondin3 induces TET2-mediated DNA hydroxymethylation of anti-inflammatory genes. a, $\alpha$-ketoglutarate ( $\alpha$-KG) to succinate ratios measured in WT, Lgr4 ${ }^{\mathrm{M} \varphi-/-}$ and Ctnnb7 ${ }^{\mathrm{M} \varphi-/-}$ BMDMs treated with Rspondin3 or LPS alone or a combination of both. Data are representative of three independent experiments, $n=3$ samples per group (mean \pm s.d.). Statistical significance was determined by two-way ANOVA with Tukey's multiple comparisons test using GraphPad Prism. $P$ values (left to right) are: ${ }^{\star \star \star \star} P<0.0001$; ${ }^{\star \star \star \star} P<0.0001 ; N S, P=0.9985 ; N S, P=0.9999 ; N S, P=0.9937 ; N S, P>0.9999 . \mathbf{b}, N u c l e a r$ extracts from WT, Lgr4 $\mathrm{M}_{\mathrm{M}-/-}, \mathrm{Ctnnnb}^{\mathrm{M} \varphi-/-}$ and Tet2 ${ }^{\mathrm{M} \varphi-/-}$ BMDMs treated with Rspondin3, LPS alone or a combination of both were used for TET activity measurement. Data are representative of three independent experiments, $n=3$ samples per group (mean \pm s.d.). Statistical significance was determined by two-way ANOVA with Tukey's multiple comparisons test using GraphPad Prism. $P$ values (left to right) are: ${ }^{\star \star \star \star} P<0.0001 ;{ }^{\star \star \star \star} P<0.0001 ;$ NS, $P=0.9988 ;$ NS, $P=0.9953 ; \mathrm{NS}, P=0.9760 ; \mathrm{NS}, P=0.9998 ; \mathrm{NS}, P>0.9999 ; \mathrm{NS} P=0.9989$. c, Genomic DNA prepared from WT, Lgr4 ${ }^{\mathrm{M} \varphi-/-}, \mathrm{Ctnnb} 7^{\mathrm{M} \varphi-/-}$ and Tet2 ${ }^{\mathrm{M} \varphi-/-} \mathrm{BMDMs}$ treated with Rspondin3, LPS alone or a combination of both were used for measurement of levels of $5 \mathrm{hmC}$. Data are representative of three independent experiments, $n=3$ samples per group (mean \pm s.d.). Statistical significance was determined by two-way ANOVA with Tukey's multiple comparisons test using GraphPad Prism. $P$ values (left to right) are: ${ }^{\star \star \star \star} P<0.0001 ;{ }^{* \star \star \star} P<0.0001 ; N S, P>0.9999 ; N S, P=0.9998 ;$ NS, $P=0.9937$; NS, $P>0.9999 ;$ NS, $P>0.9999 ;$ NS, $P>0.9999$. d, Hydroxymethylated DNA immunoprecipitation (hMeDIP) with an anti-5hmC antibody was performed using genomic DNA prepared from WT, Lgr4 ${ }^{\mathrm{M} \varphi-/-}, \mathrm{Ctnn}_{\mathrm{b}} 7^{\mathrm{M} \varphi-/-}$ and Tet2 ${ }^{\mathrm{M} \varphi-/-} \mathrm{BMDMs}$ treated with Rspondin3, LPS alone or a combination of both and qPCR with primers targeting proximal promoters of the indicated genes were used to detect enrichment of $5 \mathrm{hmC}$. Data are representative of three independent experiments, $n=3$ samples per group (mean \pm s.d.). Statistical significance was determined by two-way ANOVA with Tukey's multiple comparisons test using GraphPad Prism. $P$ values (left to right) are: Mrc1 ( ${ }^{\star \star \star \star} P<0.0001 ;{ }^{* \star \star} P=0.0004 ; N S, P>0.9999 ; N S, P>0.9999 ; N S, P>0.9999 ; N S, P>0.9999 ;$ NS, $P>0.9999 ; N S, P>0.9999)$ and $\operatorname{Arg} 1{ }^{\star \star \star \star \star} P<0.0001 ;{ }^{\star \star \star \star} P<0.0001 ; N S, P=0.9996 ; N S, P>0.9999 ;$ NS, $P>0.9999 ; N S, P>0.9999 ; N S, P>0.9999 ; N S$, $P>0.9999)$. e, ChIP with an anti-H3K4me3 antibody performed on WT, Lgr4 ${ }^{\mathrm{M} \varphi-/-}$, Ctnnb $7^{\mathrm{M} \varphi-/-}$ and Tet2 ${ }^{\mathrm{M} \varphi-/-}$ BMDMs treated with Rspondin3 or LPS alone or a combination of both and APCR with primers targeting the proximal promoters of the indicated genes were used to detect the enrichment of $\mathrm{H} 3 \mathrm{~K} 4 \mathrm{me} 3$. Data are representative of three independent experiments, $n=3$ samples per group (mean \pm s.d.). Statistical significance was determined by two-way ANOVA with Tukey's multiple comparisons test using GraphPad Prism. $P$ values (left to right) are: $\operatorname{Mrcl}\left({ }^{\star \star \star \star} P<0.0001 ;{ }^{\star \star \star \star} P<0.0001 ; N S, P>0.9999\right.$; NS, $P>0.9999 ; N S, P=0.9933 ; N S, P>0.9999 ; N S, P=0.9985 ; N S, P=0.9996)$ and Arg1 ( ${ }^{\star \star \star \star} P<0.0001 ;{ }^{\star \star \star \star} P<0.0001 ; N S, P=0.9996 ; N S, P>0.9933$; NS, $P=0.9947$; NS, $P>0.9999 ; N S, P>0.9999 ; N S, P>0.9999)$. f, Heat map of levels of anti-inflammatory markers and pro-inflammatory markers in lung IMs in WT and Tet2 ${ }^{\mathrm{M} \varphi-/-}$ mice with or without i.v. Rspondin3 under basal conditions and post-sublethal LPS challenge for $24 \mathrm{~h}$ as measured by CyTOF from three independent repeats ( $n=5$ mice per group, shown as the mean CyTOF signal intensity). $\mathbf{g}$, Lung vascular permeability measured by the EBA assay in WT and Tet $2^{\mathrm{M} \varphi-/-}$ mice with or without i.v. Rspondin3 under basal and post-sublethal LPS challenge for $24 \mathrm{~h}$ (data are representative of three independent experiments with five mice per group). Graphs show mean \pm s.d. with each dot representing an individual mouse. Statistical significance was determined by two-way ANOVA with Tukey's multiple comparisons test using GraphPad Prism and individual $P$ values (left to right) are: NS, $P>0.9215$; $* \star \star \star P<0.0001$; ${ }^{\star \star} P=0.0044$; NS, $P=0.9554$. h, MPO activity of flushed lung samples from WT and Tet2 ${ }^{\mathrm{M} \varphi-/-}$ mice with or without i.v. Rspondin3 under basal and post-sublethal LPS challenge for $24 \mathrm{~h}$ (data are representative of three independent experiments with five mice per group). Graphs show the mean \pm s.d. with each dot representing an individual mouse. Statistical significance was determined by two-way ANOVA with Tukey's multiple comparisons test using GraphPad Prism and individual $P$ values (left to right) are: NS, $P>0.9872 ;{ }^{* * * *} P<0.0001 ;{ }^{\star} P=0.0147 ; \mathrm{NS}, P=0.9134$.

In summary, we demonstrated an orchestrated angiocrinemetabolic-epigenetic signaling axis mediating the maintenance and functional regulation of lung IMs. These studies provide fundamental insights into the endothelial niche in lungs mediated by Rspondin3, which reprograms IMs to resolve inflammation and promote tissue repair following inflammatory injury. The role of Rspondin3- $\beta$-catenin-induced metabolic and epigenetic reprogramming raises the possibility of harnessing the angiocrine signaling pathway in macrophages to therapeutically target inflammatory tissue injury.

\section{Online content}

Any methods, additional references, Nature Research reporting summaries, source data, extended data, supplementary information, acknowledgements, peer review information; details of author contributions and competing interests; and statements of data and code availability are available at https://doi.org/10.1038/ s41590-020-0764-8.

Received: 3 September 2019; Accepted: 17 July 2020;

Published online: 24 August 2020

\section{References}

1. Ginhoux, F., Schultze, J. L., Murray, P. J., Ochando, J. \& Biswas, S. K. New insights into the multidimensional concept of macrophage ontogeny, activation and function. Nat. Immunol. 17, 34-40 (2016).

2. Wynn, T. A. \& Vannella, K. M. Macrophages in tissue repair, regeneration, and fibrosis. Immunity 44, 450-462 (2016).

3. Murray, P. J. \& Wynn, T. A. Protective and pathogenic functions of macrophage subsets. Nat. Rev. Immunol. 11, 723-737 (2011).

4. Salome, B. \& Magen, A. Dysregulation of lung myeloid cells in COVID-19. Nat. Rev. Immunol. 20, 277 (2020).
5. Byrne, A. J., Mathie, S. A., Gregory, L. G. \& Lloyd, C. M. Pulmonary macrophages: key players in the innate defence of the airways. Thorax $\mathbf{7 0}$, 1189-1196 (2015)

6. Lawrence, T. \& Natoli, G. Transcriptional regulation of macrophage polarization: enabling diversity with identity. Nat. Rev. Immunol. 11, 750-761 (2011).

7. Satoh, T. et al. The Jmjd3-Irf4 axis regulates M2 macrophage polarization and host responses against helminth infection. Nat. Immunol. 11, 936-944 (2010)

8. Phan, A. T., Goldrath, A. W. \& Glass, C. K. Metabolic and epigenetic coordination of $\mathrm{T}$ cell and macrophage immunity. Immunity 46, 714-729 (2017).

9. Artyomov, M. N., Sergushichev, A. \& Schilling, J. D. Integrating immunometabolism and macrophage diversity. Semin. Immunol. 28, 417-424 (2016)

10. Amit, I., Winter, D. R. \& Jung, S. The role of the local environment and epigenetics in shaping macrophage identity and their effect on tissue homeostasis. Nat. Immunol. 17, 18-25 (2016).

11. Colegio, O. R. et al. Functional polarization of tumour-associated macrophages by tumour-derived lactic acid. Nature 513, 559-563 (2014).

12. Svedberg, F. R. et al. The lung environment controls alveolar macrophage metabolism and responsiveness in type 2 inflammation. Nat. Immunol. 20, 571-580 (2019).

13. Hussell, T. \& Bell, T. J. Alveolar macrophages: plasticity in a tissue-specific context. Nat. Rev. Immunol. 14, 81-93 (2014).

14. Galli, S. J., Borregaard, N. \& Wynn, T. A. Phenotypic and functional plasticity of cells of innate immunity: macrophages, mast cells and neutrophils. Nat. Immunol. 12, 1035-1044 (2011).

15. Chakarov, S. et al. Two distinct interstitial macrophage populations coexist across tissues in specific subtissular niches. Science 363, eaau0964 (2019).

16. Sabatel, C. et al. Exposure to bacterial CpG DNA protects from airway allergic inflammation by expanding regulatory lung interstitial macrophages. Immunity 46, 457-473 (2017).

17. Ding, B. S. et al. Endothelial-derived angiocrine signals induce and sustain regenerative lung alveolarization. Cell 147, 539-553 (2011).

18. Wang, Q. et al. Vascular niche IL-6 induces alternative macrophage activation in glioblastoma through HIF-2 $\alpha$. Nat. Commun. 9, 559 (2018). 
19. Misharin, A. V., Morales-Nebreda, L., Mutlu, G. M., Budinger, G. R. \& Perlman, H. Flow cytometric analysis of macrophages and dendritic cell subsets in the mouse lung. Am. J. Resp. Cell Mol. Biol. 49, 503-510 (2013).

20. Becher, B. et al. High-dimensional analysis of the murine myeloid cell system. Nat. Immunol. 15, 1181-1189 (2014).

21. de Lau, W., Peng, W. C., Gros, P. \& Clevers, H. The R-spondin/Lgr5/Rnf43 module: regulator of Wnt signal strength. Genes Dev. 28, 305-316 (2014).

22. Glinka, A. et al. LGR4 and LGR5 are R-spondin receptors mediating Wnt/ $\beta$-catenin and Wnt/PCP signalling. EMBO Rep. 12, 1055-1061 (2011).

23. Wang, D. et al. Structural basis for R-spondin recognition by LGR4/5/6 receptors. Genes Dev. 27, 1339-1344 (2013).

24. Scholz, B. et al. Endothelial RSPO3 controls vascular stability and pruning through non-canonical WNT/Ca ${ }^{2+} / \mathrm{NFAT}$ signaling. Dev. Cell. 36, 79-93 (2016).

25. Liu, P. S. et al. $\alpha$-ketoglutarate orchestrates macrophage activation through metabolic and epigenetic reprogramming. Nat. Immunol. 18, 985-994 (2017).

26. Carey, B. W., Finley, L. W., Cross, J. R., Allis, C. D. \& Thompson, C. B. Intracellular $\alpha$-ketoglutarate maintains the pluripotency of embryonic stem cells. Nature 518, 413-416 (2015).

27. Yang, Q. et al. AMPK/ $\alpha$-ketoglutarate axis dynamically mediates DNA demethylation in the Prdm16 promoter and brown adipogenesis. Cell Metab. 24, 542-554 (2016).

28. Deplus, R. et al. TET2 and TET3 regulate GlcNAcylation and H3K4 methylation through OGT and SET1/COMPASS. EMBO J. 32, 645-655 (2013).

29. Ramasamy, S. K., Kusumbe, A. P., Wang, L. \& Adams, R. H. Endothelial Notch activity promotes angiogenesis and osteogenesis in bone. Nature 507, 376-380 (2014).

30. Keiran, N. et al. SUCNR1 controls an anti-inflammatory program in macrophages to regulate the metabolic response to obesity. Nat. Immunol. 20, 581-592 (2019).

31. Kang, S. et al. Semaphorin $6 \mathrm{D}$ reverse signaling controls macrophage lipid metabolism and anti-inflammatory polarization. Nat. Immunol. 19, 561-570 (2018).
32. Pan, W. et al. The DNA methylcytosine dioxygenase Tet 2 sustains immunosuppressive function of tumor-infiltrating myeloid cells to promote melanoma progression. Immunity 47, 284-297 (2017).

33. Goiffon, R. J., Martinez, S. C. \& Piwnica-Worms, D. A rapid bioluminescence assay for measuring myeloperoxidase activity in human plasma. Nat. Commun. 6, 6271 (2015).

34. Lambrecht, B. N. TGF- $\beta$ gives an air of exclusivity to alveolar macrophages. Immunity 47, 807-809 (2017).

35. Schyns, J. et al. Non-classical tissue monocytes and two functionally distinct populations of interstitial macrophages populate the mouse lung. Nat. Commun. 10, 3964 (2019).

36. Hume, P. S. et al. Localization of macrophages in the human lung via design-based stereology. Am. J. Respir. Crit. Care Med. 201, 1209-1217 (2020)

37. Bedoret, D. et al. Lung interstitial macrophages alter dendritic cell functions to prevent airway allergy in mice. J. Clin. Invest. 119, 3723-3738 (2009).

38. Minamino, T. \& Komuro, I. Regeneration of the endothelium as a novel therapeutic strategy for acute lung injury. J. Clin. Invest. 116, 2316-2319 (2006).

39. Tan, B. et al. Inhibition of Rspo-Lgr4 facilitates checkpoint blockade therapy by switching macrophage polarization. Cancer Res. 78, 4929-4942 (2018).

40. Du, B. et al. Lgr4/Gpr48 negatively regulates TLR2/4-associated pattern recognition and innate immunity by targeting CD14 expression. J. Biol. Chem. 288, 15131-15141 (2013).

41. Shen, Q. et al. Tet2 promotes pathogen infection-induced myelopoiesis through mRNA oxidation. Nature 554, 123-127 (2018)

42. Zhang, Q. et al. Tet2 is required to resolve inflammation by recruiting Hdac2 to specifically repress IL-6. Nature 525, 389-393 (2015).

Publisher's note Springer Nature remains neutral with regard to jurisdictional claims in published maps and institutional affiliations.

(C) The Author(s), under exclusive licence to Springer Nature America, Inc. 2020 


\section{Methods}

Mice. C57BL/6J, Lyz2-cre+ (Jax, cat. no. 019096), Rspo3 $3^{\mathrm{A} / \mathrm{fl}}$ (Jax, cat. no. 027313), Ctnnb1 $1^{\mathrm{A} / \mathrm{f}}$ (Jax, cat. no. 022775), Tet2 $2^{\mathrm{Af/1}}$ (Jax, cat. no. 017573), TCF/Lef:H2B-GFP transgenic mice (Jax, cat. no. 013752) and Rosa26-floxed STOP-Cas9 mice (Jax, cat. no. 024857) were originally purchased from Jackson Laboratory. VE-cadherin-CreERT2 mice (MGI, cat. no. 3848982) were kindly provided by R. Adams. Rspo $3^{\text {I//1/l }}$ mice were crossed with VE-cadherin-CreERT2 mice to generate

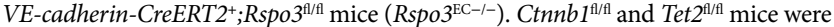
crossed with $\mathrm{Lyz}_{2-\mathrm{cre}}{ }^{+}$mice to generate $\mathrm{Lyz2} 2-\mathrm{cre}^{+} ; \mathrm{Ctnnb1}^{\mathrm{t} / \mathrm{fl}}$ mice $\left(\mathrm{Ctnnb}^{\mathrm{M} \varphi---}\right)$

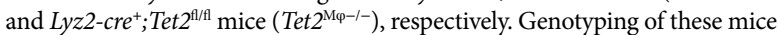
strains was either performed by regular PCR using the recommended primers on the Jackson Laboratory website (www.jax.org) followed by DNA gel imaging or by Transnetyx (Cordova) using TaqMan probe-based qPCR. All mice were housed in a temperature-controlled specific-pathogen-free facility under 12-h light-dark cycles at the University of Illinois at Chicago Animal Care Facility. Veterinary care and animal experimental procedures were approved by the University of Illinois Animal Care \& Use Committee in accordance with the guidelines of the National Institutes of Health.

Cells. Mouse BMDMs were isolated and differentiated into mature macrophages as previously described ${ }^{43}$. Mouse lung microvascular ECs were isolated, purified and cultured as previously described ${ }^{44}$. Mouse lung IMs and AMs were isolated by FACS on the MoFlo Astrios cell sorter (Beckman Coulter) with the strategy described previously ${ }^{15}$.

siRNA transfection. Mouse Lgr4 siRNA with four different targeting sites as a pool (Dharmacon, cat no. E-047291-00-0005) and nontargeting pool siRNA (Dharmacon, cat. no. D-001910-10) were transfected into BMDMs using Lipofectamine 3000 reagents (Thermo Fisher Scientific, cat. no. L3000015) as described ${ }^{45}$.

Generation of macrophage-specific $\operatorname{Lgr} 4$ knockout $\left(\operatorname{Lgr} 4^{\mathrm{M} \varphi-l-}\right)$ mice. $\operatorname{Lgr} 4^{\mathrm{M} \varphi-/}$ mice were generated using the CRISPR-Cas9 strategy. Briefly, a transgenic mouse strain with Cas9-specific expression in myeloid cells under control by Lyz2-cre ${ }^{+}$ was generated by crossing Rosa26-floxed STOP-Cas 9 mice with $L y z 2-c r e^{+}$mice as described $^{25}$, then single guide RNA targeting three different sites (as a pool) for Lgr4 (IDT, cat. no. Mm.Cas9.LGR4.1.AA; IDT, cat. no. Mm.Cas9.LGR4.1.AB; IDT, cat. no. Mm.Cas9.LGR4.1.AC) was packaged in liposome using Invivofectamine 3.0 reagent (Thermo Fisher Scientific) and delivered into mice via an i.v. tail vein injection ( $10 \mathrm{nmol}$ per mouse) to achieve macrophage uptake by phagocytosis in vivo ${ }^{46}$.

Recombinant proteins. Recombinant murine Rspondin 3 was purchased from RDsystem (cat. no. 4120-RS-025). Recombinant M-CSF was purchased from PeproTech (cat. no. 315-02)).

Acute lung injury models. Two ALI mice models were used in this study. The first model is the widely used classical LPS-induced ALI model in which mice are systemically challenged with sublethal LPS $\left(12 \mathrm{mg} \mathrm{kg}^{-1} \text { i.p. }\right)^{43,44,47}$. The second inflammatory lung injury model is the bleomycin model, which has also been previously described ${ }^{15,48}$. Briefly, mice were anesthetized with isoflurane, their lungs were intubated orally with a 20-gauge Angiocath (Franklin Lanes) and $0.025 \mathrm{IU}$ bleomycin (Thermo Fisher Scientific, cat. no. AAJ67560SDL) in $50 \mu \mathrm{l}$ of sterile of PBS were instilled through the catheter. Mice were killed $5 \mathrm{~d}$ (ALI phase) after the instillation of bleomycin for further analysis.

RNA isolation and real-time qPCR. Total RNA from BMDMs or lung ECs was isolated using the PureLink RNA kit (Thermo Fisher Scientific, cat. no. $12183018 \mathrm{~A}$ ) and were reverse-transcribed into complementary DNA using iScript reverse transcription supermix (Bio-Rad, cat. no. 1708840) according to manufacturer's instructions. The cDNA obtained was mixed with PowerUp SYBR Green Master Mix (Thermo Fisher Scientific, cat. no. A25742) with specific qPCR primers (Supplementary Table 2) for qPCR on an ABI Prism 7000 system and analyzed with QuantStudio software v.1.3 (Thermo Fisher Scientific). Heat maps for the change folds of gene levels were generated by Morpheus (https://software. broadinstitute.org/morpheus).

Chromatin immunoprecipitation. ChIP was performed with cross-linked chromatin from mouse BMDMs using a SimpleChIP plus sonication Chromatin IP kit (CST, cat. no. 56383) with ChIP grade H3K4me3 antibody (CST, cat. no. 9751) with a dilution ratio of 1:500 according to the manufacturer's instructions. The GPCR following ChIP was performed with the primers listed in Supplementary Table 2 .

Lung vascular permeability and inflammation measurements. Lung vascular permeability was measured by the EBA dye tracer and lung inflammation was evaluated by MPO activity as previously described ${ }^{43,44}$.

Preparation of mouse lung single-cell suspensions. Mice were anesthetized with ketamine/xylazine and killed, followed by perfusing with $10 \mathrm{ml}$ of PBS via the right ventricle. The lungs were removed and cut into tiny pieces with scissors, followed by incubation with digestion buffer $\left(1 \mathrm{mg} \mathrm{ml}^{-1}\right.$ of collagenase IV and $0.1 \mathrm{mg} \mathrm{ml}^{-1}$ DNase I, both from Millipore-Sigma) in a shaking water bath $\left(37^{\circ} \mathrm{C}\right)$ for $30 \mathrm{~min}$. The digested lung pieces were passed through a $20-\mathrm{G}$ needle ten times and filtered by a $40-\mu \mathrm{m}$ nylon mesh to obtain a single-cell suspension. The remaining red blood cells were lysed using RBC lysis buffer (BioLegend, cat no. 420301). The acquired lung single-cell suspensions were used for the followed applications such as flow cytometry, CyTOF analysis and cell sorting.

Flow cytometry. For BMDMs, flow cytometry was performed with anti-mouse CD11b, F4/80, CD64 to gate-matured macrophages. Mouse macrophage anti-inflammatory markers CD206, CD301, IL-10 and arginase 1 and pro-inflammatory markers CD86, CD80, iNOS and TNF were stained with the fluorophore-coupled antibodies listed in Supplementary Table 3. For mouse lung macrophages, flow cytometry was performed to identify lung macrophage subpopulations ${ }^{19}$. Briefly, cells were stained with anti-mouse CD45, CD11b, CD64, Ly6G, SiglecF, CD86 and CD206 antibodies listed in Supplementary Table 3 using an eBioscience flow cytometry staining buffer (Thermo Fisher Scientific, cat. no. 00-4222-26) according to the manual. Samples were run on a CytoFLEX S Flow Cytometer (Beckman) and data were analyzed by Kaluza Analysis 2.1 software (Beckman).

\section{Cytometry by time-of-flight mass spectrometry. CyTOF allows for} high-dimensional analysis of cell surface markers, cytokines and signaling molecules simultaneously at the single-cell level ${ }^{20}$. Thus, we used the CyTOF for phenotyping and function assay for the mouse lung myeloid populations including IMs, AMs, monocytes, neutrophils, DCs and eosinophils. A panel of metal-labeled antibodies (Supplementary Table 4), including several markers for macrophages and myeloid cells as well as cytokines and signaling molecules (CD45, CD11b, F4/80, CD64, Ly6C, Ly6G, MerTK, CD24, CD206, SiglecF, CD11c, CD301, arginase 1, LGR4, $\beta$-catenin, IL-10, iNOS, TNF, CX3CR1, CD80, CD69, CD86, CCR2, CD115, I-A/I-E, BST2, RELM- $\alpha$, CD103, CD3, CD49 and TER-119) were used for staining according to the FLUIDIGM recommended protocol. Samples were run on the Helios CyTOF mass cytometer (FLUIDIGM) at the flow cytometry core of the Research Resources Center of the University of Illinois at Chicago. Data from CyTOF were analyzed using the Cytobank online analysis tool (www.cytobank.org).

Metabolism assays. Macrophages (BMDMs) prepared from the indicated mouse strains were uniformly plated in XF96 plates overnight and treated with PBS, Rspondin3, LPS alone or LPS and Rspondin3 together for $24 \mathrm{~h}$. Metabolic profiling was performed with the Seahorse XF96 extracellular flux analyzer (Agilent) with Wave 2.6.1 software (Agilent) as previously described ${ }^{49}$. Experiments were conducted in XF medium (nonbuffered DMEM containing $10 \mathrm{mM}$ glucose, $2 \mathrm{mM}$ glutamine and $2 \mathrm{mM}$ sodium pyruvate), with OCR measured basally and in response to sequential addition of oligomycin, FCCP and rotenone plus antimycin A. Fuel preference was measured after $1 \mathrm{~h}$ of incubation in Seahorse XF basal medium (Agilent) with no fuel substrates and then immediately before the assay supplemented with either $10 \mathrm{mM}$ glucose, $4 \mathrm{mM}$ glutamine or $10 \mathrm{nM}$ free fatty acids in the form of palmitate BSA. All Seahorse XF data were normalized to an equal cell number of $1 \mathrm{mg}$ total protein.

ELISA. Supernatants were collected from BMDMs under different conditions as indicated in figure legends and the cytokines IL- 10 and IL- $1 \beta$ were measured using mouse IL-10 DuoSet ELISA (RDsystem, cat. no. DY417) or mouse IL-1 $\beta$ DuoSet ELISA (RDsystem, cat. no. DY401), respectively. Secreted Rspondin3 in cultured lung ECs was measured using mouse Rspondin 3 DuoSet ELISA (RDsystem, cat. no. DY4120-005) with the supernatants collected. Optical density data were collected on a Synergy HTX multimode microplate reader (BioTek) with Gen5 software (BioTek). And cell numbers in each well of the cell culture plate were counted using a CyQuant kit (Thermo Fisher Scientific, cat. no. C7026). Calculated levels of cytokines were adjusted with cell number.

Immunoblotting. Protein samples for lung ECs and non-ECs were prepared and immunoblots were performed as previously described ${ }^{44}$. Polyclonal anti-RSPO3 (Abclonal, A8389) with a dilution of 1:3,000 and monoclonal anti- $\beta$-actin (8H10D10) mouse (CST, cat. no. 3700) with a dilution of 1:5,000 were used for immunoblotting.

Angiocrine factor profiling. Proteomics-based global secretome analysis was performed by the mass spectrometry core of Research Resources Center in University of Illinois at Chicago using EC-conditioned medium as previously described ${ }^{50}$.

Immunofluorescence staining and proximity ligation assay. Monoclonal anti-TET2 rabbit (CST, cat. no. 36449) and monoclonal anti- $\beta$-catenin mouse (Thermo Fisher Scientific, cat. no. 13-8400) with a dilution of 1:500 were used as primary antibodies. An AF488-conjugated donkey anti-mouse IgG and an AF594-conjugated goat anti-rabbit IgG, used as secondary antibodies with a dilution of 1:2,000, were used for immunofluorescence staining following the CST immunofluorescence general protocol (https://www.cellsignal.com/contents/ 
resources-protocols/immunofluorescence-general-protocol/if). Images were taken with the Zeiss LSM880 confocal microscope with Zen software 3.1 (Zeiss) and analyzed with ImageJ software v.1.52p. Proximity ligation assays (PLAs) for detecting protein interactions with high specificity and sensitivity were performed with Duolink In Situ Red Starter Kit Mouse/Rabbit (Millipore-Sigma, cat. no. DUO92101) using the above monoclonal anti-TET2 rabbit and monoclonal anti- $\beta$-catenin mouse as validated by immunofluorescence staining according to the standard manual ${ }^{51}$. Briefly, monoclonal anti-TET2 rabbit and monoclonal anti- $\beta$-catenin mouse were first used to detect TET2 and $\beta$-catenin on macrophages, then a pair of PLA probes (anti-rabbit plus and anti-mouse minus) were used as secondary antibodies; next, hybridizing connector oligonucleotides joined the PLA probes only if they were in close proximity to each other and ligase formed a closed circle DNA template for generating rolling-circle amplification that gives red fluorescence.

Assay for specific enrichment of 5 hmC DNA fragments. To detect the enrichment of $5 \mathrm{hmC}$ DNA fragments, genomic DNA was isolated from macrophages and hMeDIP was performed using a highly specific purified $5 \mathrm{hmC}$ antibody with a dilution ratio of 1:500 contained by an hMeDIP kit purchased from ActiveMotif (cat. no. 55010) as reported ${ }^{52}$. Real-time qPCR was performed using $5 \mathrm{hmC}$ antibody precipitated DNA with specific primers on the proximal promoter regions of genes as listed in Supplementary Table 2.

$\alpha$-ketoglutarate and succinate measurements and glutaminase activity assay The levels of $\alpha$-ketoglutarate and succinate in macrophages were measured by $\alpha$-Ketoglutarate Colorimetric/Fluorometric Assay kit (BioVision, cat. no. K677) and Succinate (Succinic Acid) Colorimetric Assay kit (BioVision, cat. no. K649), respectively as previously described ${ }^{25}$. Glutaminase activity was measured using the PicoProbe Glutaminase (GLS) Activity Assay kit (Fluorometric) (BioVision, cat. no. K455) according to the manual, as previously described ${ }^{53}$.

5mC-hydroxylase TET activity and JMJD3 demethylase activity assay. The activities of 5mC-hydroxylase TET and JMJD3 demethylase were measured using the Epigenase 5mC-Hydroxylase TET Activity Assay kit (Epigentek, cat. no. P-3086) and Epigenase JMJD3 Demethylase Activity kit (Epigentek, cat. no. P-3084), respectively according to the manual, as previously reported ${ }^{26,54}$. Briefly, nuclear extracts were prepared from macrophages with the EpiQuik Nuclear Extraction kit (Epigentek, cat. no. OP-0002), then $5 \mu \mathrm{g}$ of nuclear protein from each sample was used to incubate with the TET or JMJD3 demethylase substrates respectively, the catalytic activity was measured by the absorbance on a microplate reader (BioTek) with $10 \mathrm{~min}$ at $450 \mathrm{~nm}$ with a reference wavelength of $655 \mathrm{~nm}$ with Gen 5 software (BioTek). The TET or JMJD3 activity was represented by optical density $\mathrm{min}^{-1} \mathrm{mg}^{-1}$.

Global DNA hydroxymethylation assay. Global $5 \mathrm{hmC}$ levels were measured using the MethylFlash Global DNA Hydroxymethylation (5-hmC) ELISA kit (Epigentek, cat. no. P-1032) according to the manual, as described ${ }^{26}$. Briefly, genomic DNA was isolated from macrophages from the indicated groups and $1 \mu \mathrm{g}$ of DNA was bound to strip-wells that have a high DNA affinity included in the kit. Then the hydroxymethylated fraction of DNA was detected using a $5 \mathrm{hmC}$ monoclonal antibody-based detection complex in a one-step manner and then quantified colorimetrically by reading the absorbance on a microplate spectrophotometer (BioTek) at $450 \mathrm{~nm}$ with Gen5 software (BioTek). The percentage of $5 \mathrm{hmC}$ was calculated using the formula generated from an accompanied standard curve.

Statistical analysis. Statistical significance was analyzed using Prism v.8 (GraphPad Software) with tests as described in figure legends. All experiments were repeated independently at least three times. Sample size and $P$ values are all cited in the figures and their captions.

Reporting Summary. Further information on research design is available in the Nature Research Reporting Summary linked to this article.

\section{Data availability}

Source data are provided with this paper. Other data that support the findings of this study are available from the corresponding author upon request.

\section{References}

43. Di, A. et al. The TWIK2 potassium efflux channel in macrophages mediates NLRP3 inflammasome-induced inflammation. Immunity 49, 56-65 (2018).

44. Liu, M. et al. Sox17 is required for endothelial regeneration following inflammation-induced vascular injury. Nat. Commun. 10, 2126 (2019).

45. Nepal, S. et al. STAT6 induces expression of Gas6 in macrophages to clear apoptotic neutrophils and resolve inflammation. Proc. Natl Acad. Sci. USA 116, 16513-16518 (2019).

46. Luo, Y. L. et al. Macrophage-specific in vivo gene editing using cationic lipid-assisted polymeric nanoparticles. ACS Nano. 12, 994-1005 (2018).

47. Ficz, G. et al. Dynamic regulation of 5-hydroxymethylcytosine in mouse ES cells and during differentiation. Nature 473, 398-402 (2011).

48. Sorensen, I., Adams, R. H. \& Gossler, A. DLL1-mediated Notch activation regulates endothelial identity in mouse fetal arteries. Blood 113, 5680-5688 (2009).

49. Nelson, V. L. et al. PPAR $\gamma$ is a nexus controlling alternative activation of macrophages via glutamine metabolism. Genes Dev. 32, 1035-1044 (2018).

50. Blanco, M. A. et al. Global secretome analysis identifies novel mediators of bone metastasis. Cell Res. 22, 1339-1355 (2012).

51. Fredriksson, S. et al. Protein detection using proximity-dependent DNA ligation assays. Nat. Biotechnol. 20, 473-477 (2002).

52. Takayama, K. et al. TET2 repression by androgen hormone regulates global hydroxymethylation status and prostate cancer progression. Nat. Commun. 6, 8219 (2015)

53. Wang, J. B. et al. Targeting mitochondrial glutaminase activity inhibits oncogenic transformation. Cancer Cell 18, 207-219 (2010).

54. Thienpont, B. et al. Tumour hypoxia causes DNA hypermethylation by reducing TET activity. Nature 537, 63-68 (2016).

\section{Acknowledgements}

The studies were supported by National Institutes of Health grants R01-HL45638 (to A.B.M.), P01-HL60678 (to A.B.M. and J.R.), T32-HL007829 (to A.B.M.), R01-HL118068 (to J.R. and A.B.M.) and R01-HL90152 (to J.R. and A.B.M.). The VE-Cadherin-CreERT2 mice were kindly provided by R. Adams at the Max Planck Institute.

\section{Author contributions}

B.Z., J.R. and A.B.M. designed the experiments and evaluated the data. B.Z., L.M., Z.H., L.S.H., S.C., Y.T., C.H., L.W., A.D., B.G. and X.G. performed the experiments. B.Z., J.R. and A.B.M. conceived the project and directed the research. B.Z., J.R. and A.B.M. wrote the manuscript.

\section{Competing interests}

The authors declare no competing interests.

\section{Additional information}

Supplementary information is available for this paper at https://doi.org/10.1038/ s41590-020-0764-8.

Correspondence and requests for materials should be addressed to J.R. or A.B.M.

Peer review information Zoltan Fehervari was the primary editor on this article and managed its editorial process and peer review in collaboration with the rest of the editorial team.

Reprints and permissions information is available at www.nature.com/reprints. 
a

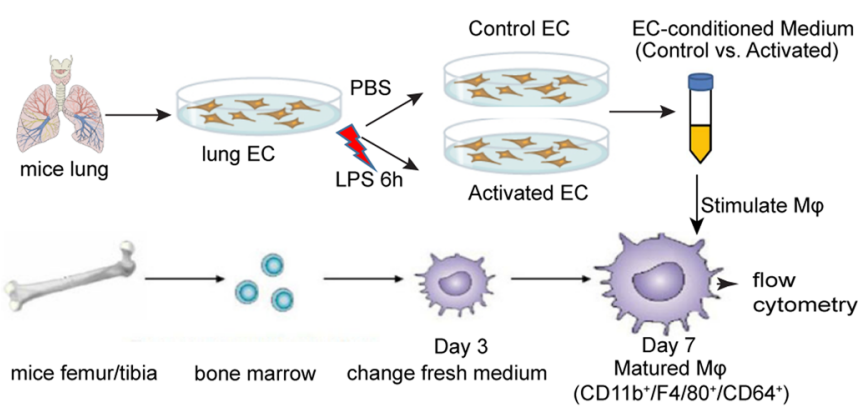

d

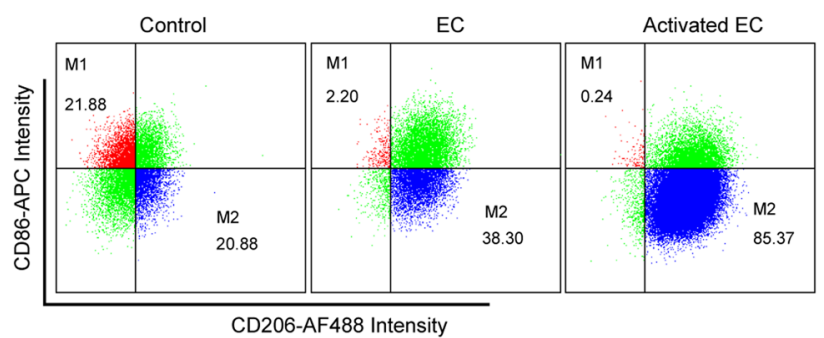

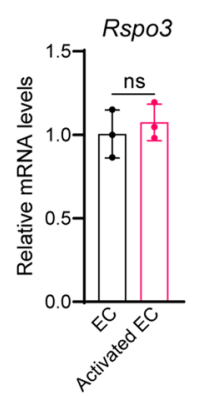

C

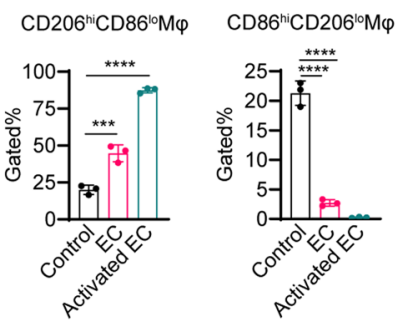

e

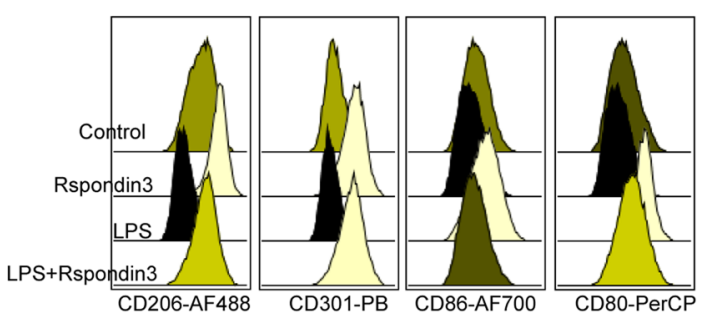

f
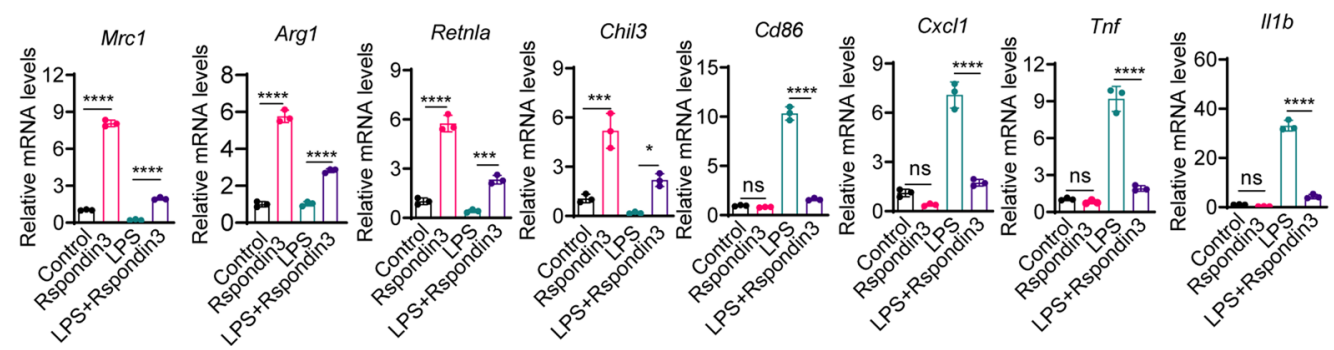

Extended Data Fig. 1 | Endothelial cells instruct macrophage phenotype transition via Rspondin3. a, Schematic of investigation of the angiocrine effects of ECs on macrophages; $\mathbf{b}$, mRNA levels of Rspo3 in ECs with or without activated by LPS was measured by qPCR; $n=3$ samples per group ( $m e a n \pm s d)$, two-sided unpaired Student's t-test was determined using GraphPad Prism. ns $P=0.5417$. c, Alternatively activated (M2) and classically activated (M1) macrophages measured by flow cytometry from three independent experiments with $n=3$ samples per group (mean $\pm \mathrm{sd}$ ); Statistical significance was determined by one-way ANOVA with Tukey's multiple comparisons test using GraphPad Prism with individual P values (left to right) are ${ }^{\star \star \star} P=0.0006,{ }^{\star \star \star \star} P<0.0001,{ }^{\star \star \star \star} P<0.0001,{ }^{\star \star \star \star *} P<0.0001$. d, Representative flow cytometry plots from three independent repeats showing ratios of $\mathrm{M} 1\left(\mathrm{CD} 86^{\mathrm{hi}} \mathrm{CD} 206^{10}\right)$ and $\mathrm{M} 2\left(\mathrm{CD} 206^{\mathrm{hi}} \mathrm{CD} 86^{10}\right)$ populations in BMDMs treated with control medium, EC-conditioned medium (EC), or activated EC conditioned medium (Activated EC) for 24h, respectively; e, Represented overlaid flow cytometry histograms from three independent repeats showing the MFI of the anti-inflammatory markers (CD206, CD301) and pro-inflammatory markers (CD86, CD80) in BMDMs stimulated with Rspondin3, LPS alone or in combination for 24h; $\mathbf{f}$, Gene expression levels of anti-inflammatory maker genes (Mrc1, Arg1, Retnla, Chil3) and pro-inflammatory marker genes (Cd86, Cxcl1, I17b, Tnf) as measured by qPCR in BMDMs treated with Rspondin3, LPS alone or in combination for 24h from three independent experiments with $\mathrm{n}=3$ samples per group (mean $\pm \mathrm{sd}$ ); Statistical significance was determined by one-way ANOVA with Tukey's multiple comparisons test using GraphPad

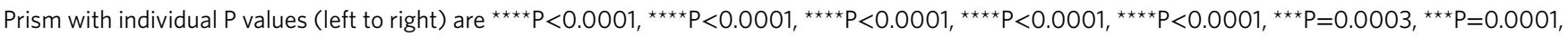
${ }^{\star} \mathrm{P}=0.0126$, ns: $\mathrm{P}=0.9486$, ${ }^{\star \star \star *} \mathrm{P}<0.0001$, ns $\mathrm{P}=0.2351$, ${ }^{\star \star \star \star} \mathrm{P}<0.0001$, ns $\mathrm{P}=0.9462,{ }^{\star \star \star * \star} \mathrm{P}<0.0001, \mathrm{~ns} \mathrm{P}=0.8783$, ${ }^{\star \star \star \star} \mathrm{P}<0.0001$. 

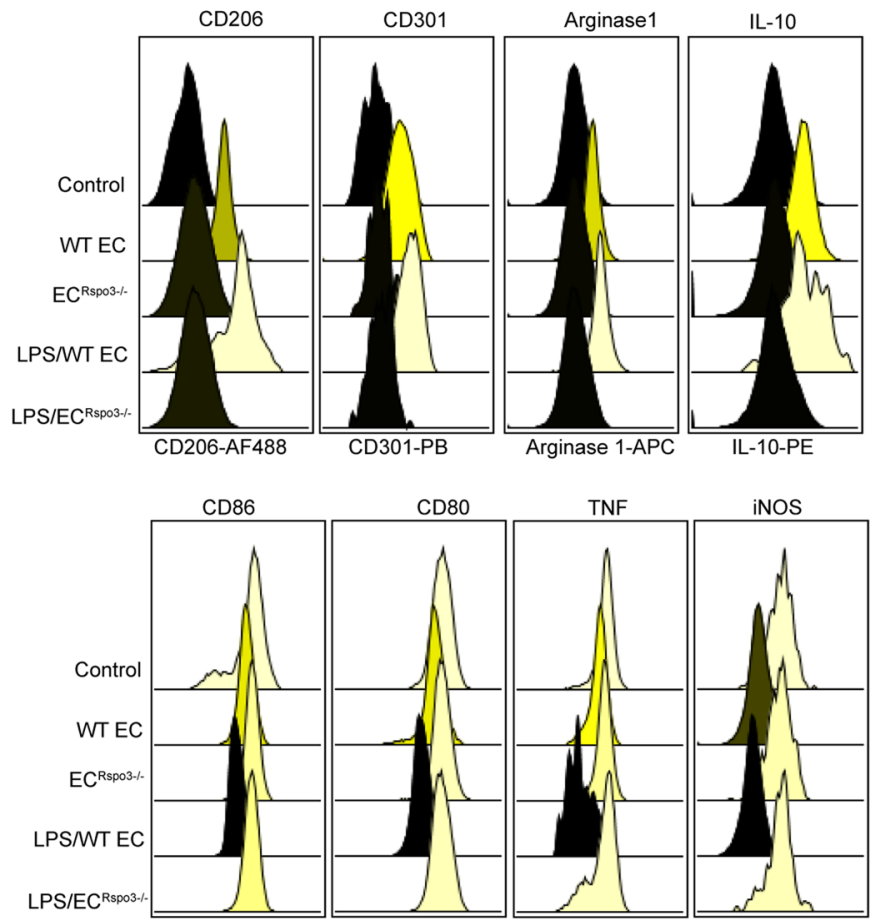

b
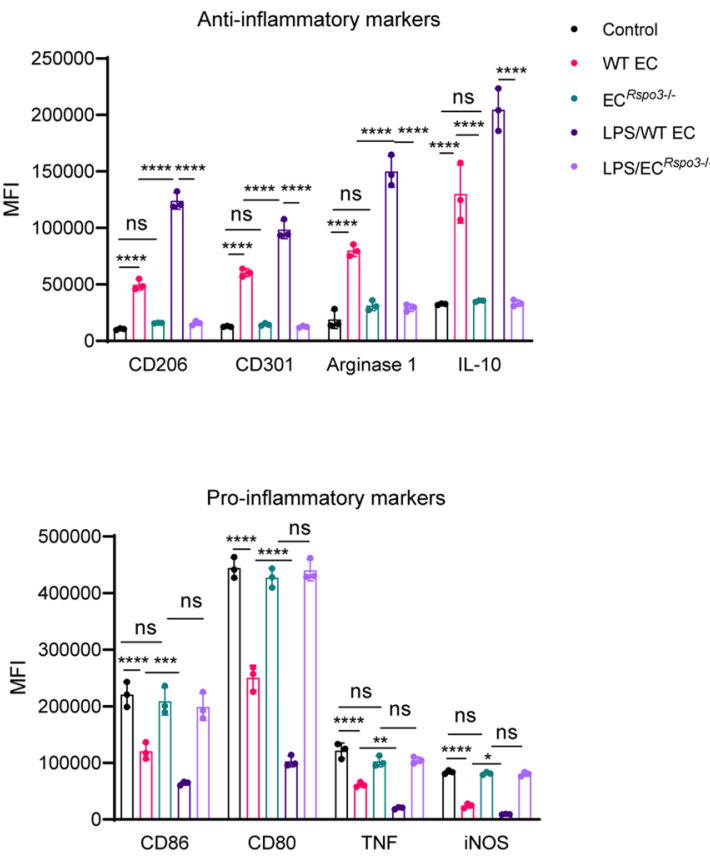
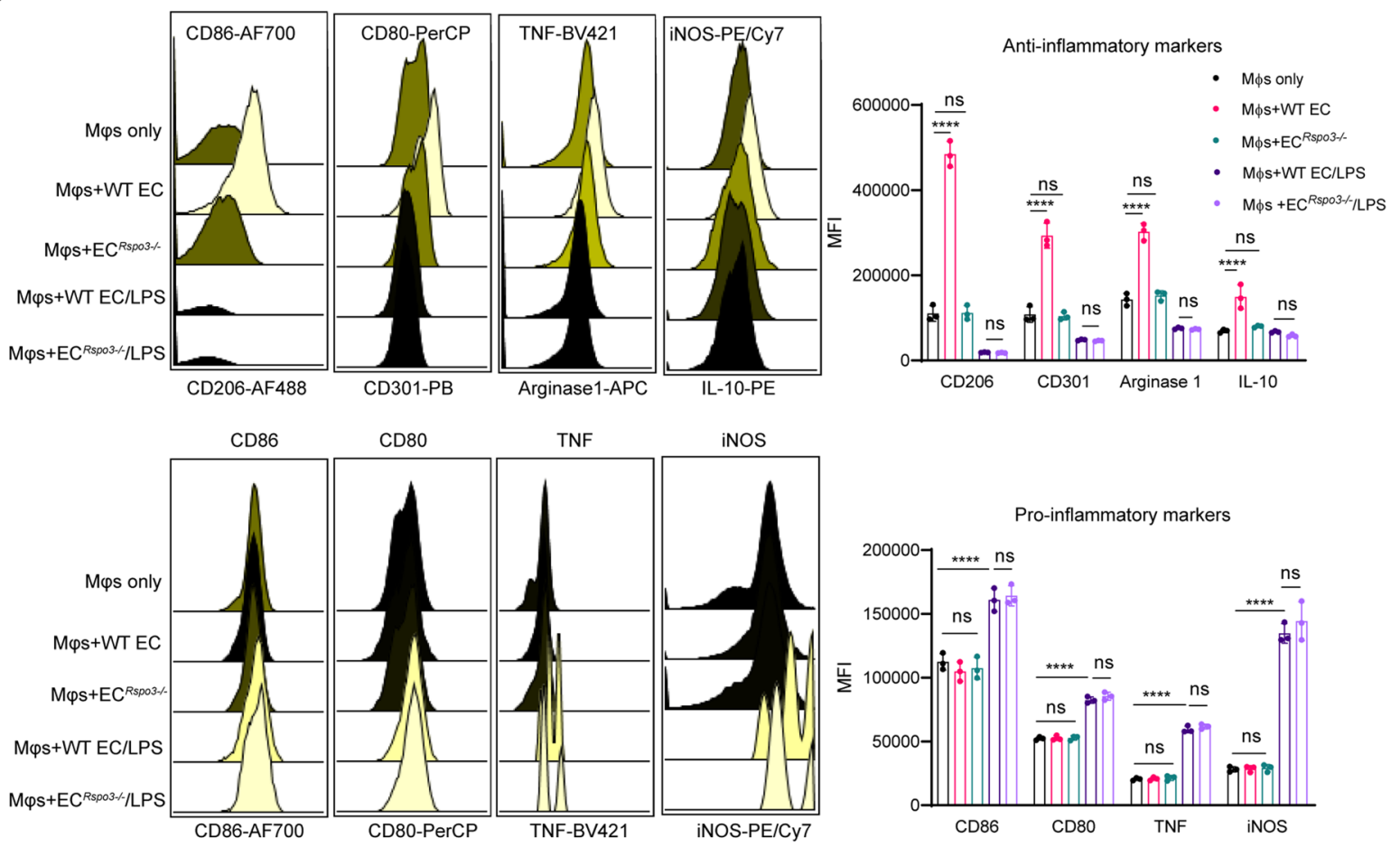

Extended Data Fig. 2 | See next page for caption. 
Extended Data Fig. 2 | Rspondin3 regulates macrophage phenotype transition via an angiocrine manner. a, Levels of anti-inflammatory markers (CD206, CD301, arginase 1, IL-10) and pro-inflammatory markers (CD86, CD80, TNF, iNOS) measured by flow cytometry in BMDMs incubated with the EC conditioned medium collected from lung ECs isolated from wildtype mice (WT EC), Rspo3 ${ }^{\mathrm{EC}-}$ - mice (EC ${ }^{\text {sspo3-/ }}$ ) at baseline or endotoxemia conditions (LPS i.p., $12 \mathrm{mg} / \mathrm{kg}$ for 24h) (LPS/WT EC, LPS/ EC Rspo3-/ ), respectively; Data are representative of three independent experiments with $\mathrm{n}=3$ samples per group (mean $\pm \mathrm{sd}$ ), statistical significance was determined by two-way ANOVA with Tukey's multiple comparisons test using GraphPad Prism with individual $P$ values (left to right) are: $C D 206$ ( $\left.{ }^{\star \star \star \star} P<0.0001, n s P=0.9405,{ }^{\star \star \star} P=0.0002,{ }^{\star \star \star \star \star} P<0.0001\right), C D 301{ }^{(\star \star \star \star} P<0.0001, n s P=0.9991,{ }^{\star \star \star \star \star} P<0.0001$, $\left.{ }^{\star \star \star \star \star} \mathrm{P}<0.0001\right)$, arginase1 ( $\left.{ }^{\star \star \star \star} \mathrm{P}<0.0001, \mathrm{~ns} \mathrm{P}=0.4164,{ }^{\star \star \star \star} \mathrm{P}<0.0001,{ }^{\star \star \star \star \star} \mathrm{P}<0.0001\right), \mathrm{IL}-10\left({ }^{\star \star \star \star} \mathrm{P}<0.0001, \mathrm{~ns} \mathrm{P}=0.9933\right.$, $\left.{ }^{\star \star \star \star} \mathrm{P}<0.0001,{ }^{\star \star \star \star \star} \mathrm{P}<0.0001\right)$,

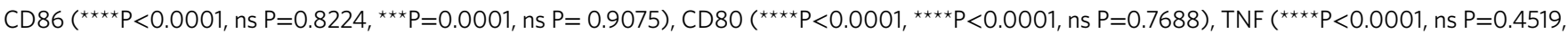
${ }^{\star} \mathrm{P} P=0.0061$, ns $\mathrm{P}=0.9997$ ), iNOS ( ${ }^{\star \star * \star} \mathrm{P}<0.0001$, ns $\mathrm{P}=0.9996$, ns $\mathrm{P}=0.6726$, ns $\left.P>0.9999\right)$. b, Levels of anti-inflammatory markers (CD206, CD301, arginase 1, IL-10) and pro-inflammatory markers (CD86, CD80, TNF, iNOS) measured by flow cytometry in BMDMs co-cultured in-contact with the

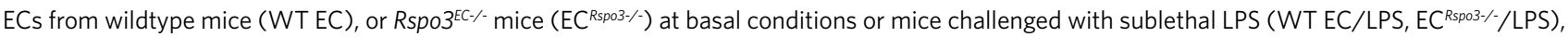
respectively; Data are representative of three independent experiments with $n=3$ samples per group (mean $\pm s$.d), with each dot representing an individual sample. Statistical significance was determined by two-way ANOVA with Tukey's multiple comparisons test using GraphPad Prism with individual P values

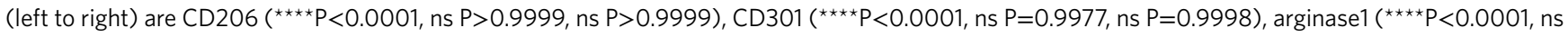
$P=0.9328$, ns $P=0.9998$ ), IL-10 ( ${ }^{\star \star \star \star} P<0.0001$, ns $P=0.8989$, ns $P=0.9393$ ), CD86 (ns $P=0.8308$, ${ }^{\star \star \star \star ~} P<0.0001, n s P=0.9600$ ), $C D 80$ (ns $P>0.9999$, ${ }^{\star \star \star \star} P<0.0001$, ns $P=0.9679$ ), TNF (ns $P=0.9997$, ${ }^{\star \star \star \star} P<0.0001$, ns $P=0.9903$ ), iNOS (ns $P=0.9996$, ${ }^{\star \star \star \star} P<0.0001$, ns $P=0.2748$ ). 
a
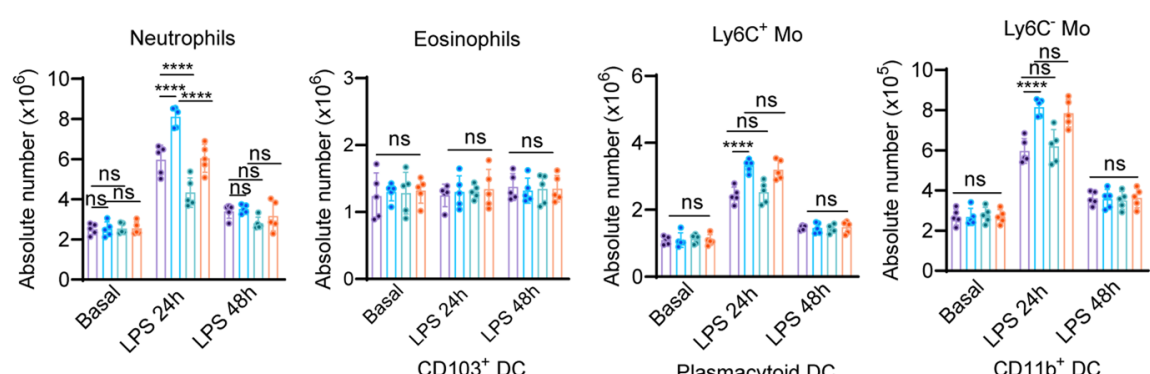

- $\mathrm{WT}$

$\mathrm{CD} 103^{+} \mathrm{DC}$
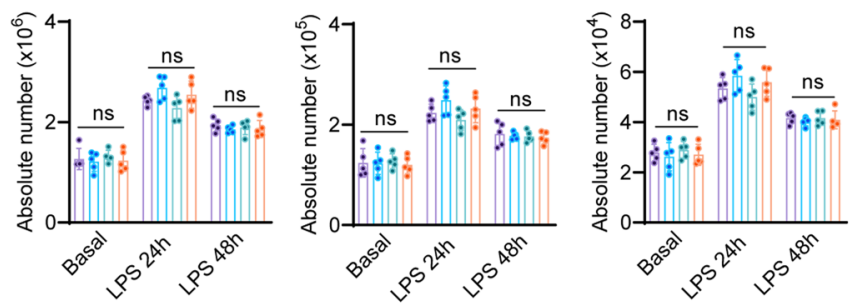

b
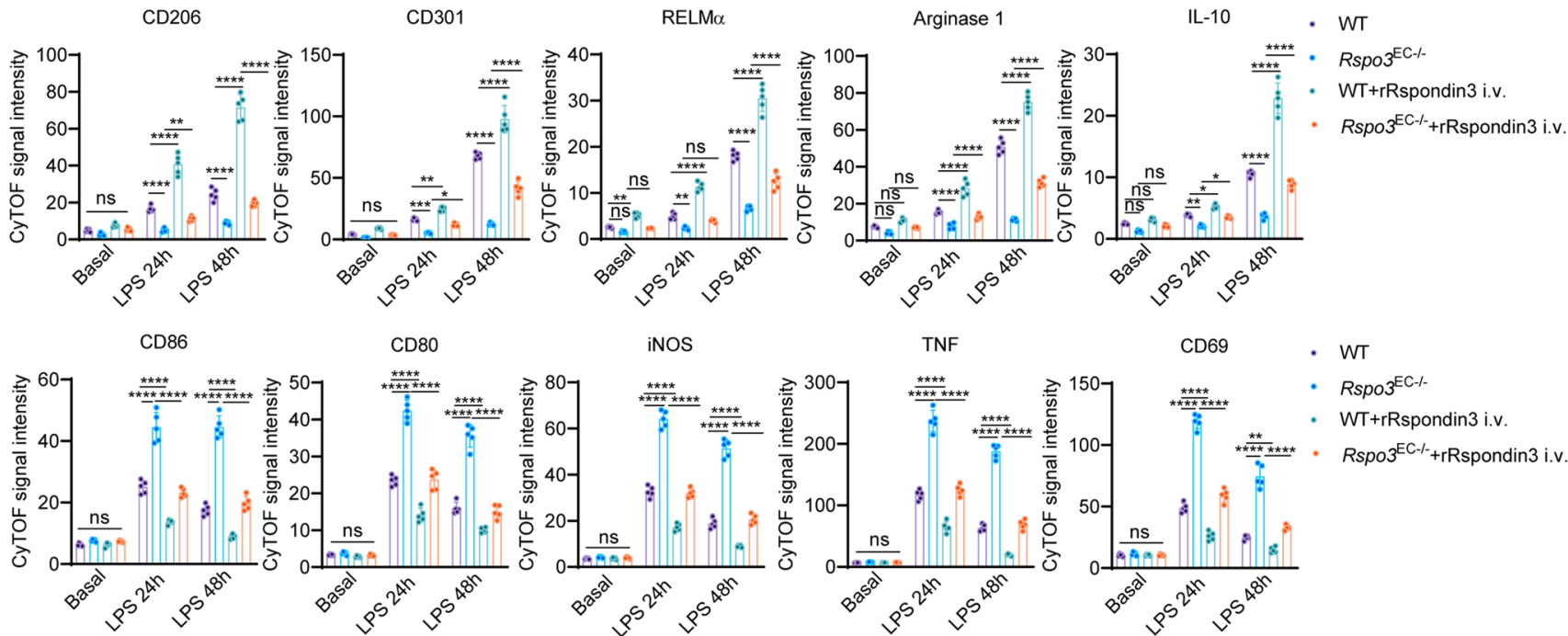

C

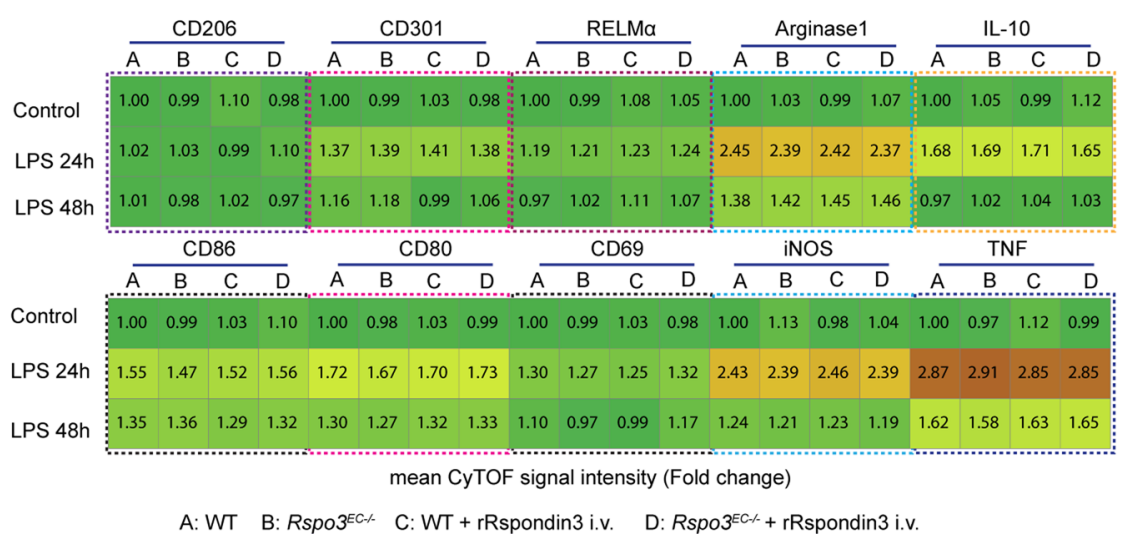

d

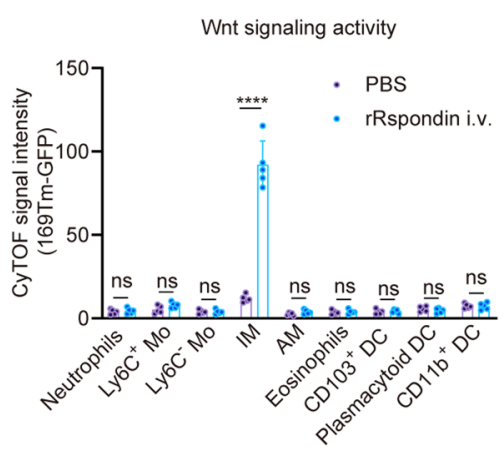

Extended Data Fig. 3 | See next page for caption. 
Extended Data Fig. 3 | Rspondin3 regulates lung interstitial macrophage phenotype transition following acute lung injury. a, Absolute cell number of lung myeloid cells including neutrophils, eosinophils, Ly6C+Mo, Ly6C- Mo, CD103+ DC, plasmacytoid DC and CD11b+ DC in WT and Rspo3 EC- - mice with or without Rspondin3 i.v. under baseline conditions and post sublethal LPS challenge for $24 \mathrm{~h}$ or $48 \mathrm{~h}$ as measured by CyTOF (data are representative of three independent experiments with $n=5$ mice per group). Graphs show the mean \pm s.d, with each dot representing an individual mouse. Statistical significance was determined by two-way ANOVA with Tukey's multiple comparisons test using GraphPad Prism with individual $P$ values (left to right) are: neutrophils (ns $P>0.9999$, ns $P=0.9995$, ns $P=0.9991$, ${ }^{\star \star \star \star} P<0.0001$, ${ }^{\star \star \star \star *} P<0.0001$, ${ }^{\star \star \star \star} P<0.0001$, ns $P=0.9864, n s P=0.2420$, ns $P=0.6898$ ), eosinophils (ns $P>0.9710$, ns $P>0.9789$, ns $P>0.9865$ ), Ly6C+Mo (ns $P>0.9933$, ${ }^{\star \star \star \star *} P<0.0001$, ns $P=0.8936$, ns $P=0.7754$, ns $P>0.9878$ ), Ly6C- Mo (ns $P>0.9942,{ }^{\star \star \star \star *} P<0.0001$, ns $P=0.9059$, ns $P=0.7988$, ns $P>0.9807$ ), $C D 103^{+} D C$ (ns $P>0.9676$, ns $P>0.1488$, ns $P>0.8806$ ), plasmacytoid $D C$ (ns $P>0.9445$, ns $P>0.2290$, ns $P>0.9786$ ), CD11b+ DC (ns $P>0.9377$, ns $P>0.2463$, ns $P>0.9941$ ); $\mathbf{b}$, Levels of anti-inflammatory markers (CD206, CD301, RELM $\alpha$, arginase 1, IL-10) and pro-inflammatory markers (CD86, CD80, iNOS, TNF, CD69) in lung IM in WT and Rspo3 $3^{\mathrm{EC}-}$ mice with or without Rspondin3 i.v. under basal and post sublethal LPS challenge for $24 \mathrm{~h}$ or $48 \mathrm{~h}$ measured by CyTOF (data are representative of three independent experiments with five mice per group). Graphs show the mean \pm s.d, with each dot representing an individual mouse. Statistical significance was determined by two-way ANOVA with Tukey's multiple comparisons test using GraphPad Prism with individual P values (left to right) are: CD206 (ns $P>0.4229$, **** $P<0.0001$,

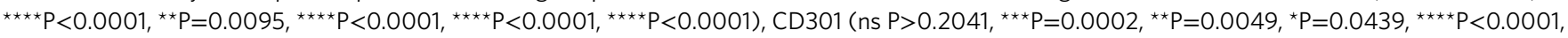

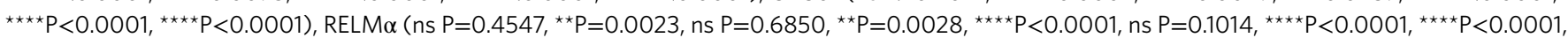
${ }^{\star \star \star \star \star} P<0.0001$ ), arginase 1 (ns $P=0.1315$, ns $P=0.0640$, ns $P=0.2260$, ${ }^{\star \star \star \star \star} P<0.0001,{ }^{\star \star \star \star \star} P<0.0001,{ }^{\star \star \star \star} P<<0.0001,{ }^{\star \star \star \star \star} P<0.0001,{ }^{\star \star \star \star \star} P<0.0001$, ${ }^{\star \star \star \star \star} P<0.0001$ ), IL-10 (ns $P=0.0847$, ns $P=0.5465$, ns $P=0.3525$, ${ }^{\star \star} P=0.0037,{ }^{\star} P=0.0448,{ }^{\star} P=0.0217,{ }^{\star \star \star \star} P<0.0001,{ }^{\star \star \star \star \star} P<0.0001,{ }^{\star \star \star \star \star} P<0.0001$ ),

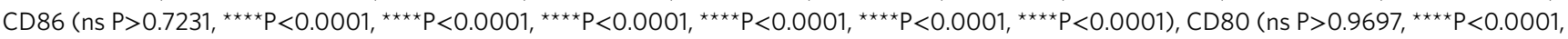

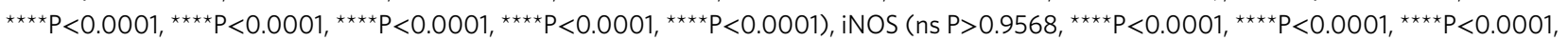

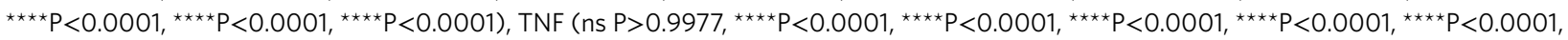

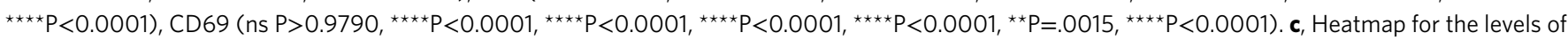
anti-inflammatory markers (CD206, CD301, RELM $\alpha$, arginase 1, IL-10) and pro-inflammatory markers (CD86, CD80, iNOS, TNF, CD69) in lung AM in WT and Rspo3EC/ mice with or without Rspondin3 i.v. under baseline conditions and post sublethal LPS challenge for 24h or 48h as measured by CyTOF (Data are representative of three independent experiments with $n=5$ mice per group, shown as fold change by the mean CyTOF signal intensity normalized to Control group); d, Wnt signaling activities in mice lung myeloid populations determined by CyTOF using Wnt reporter mice (TCF/Lef:H2B-GFP transgenic mice) with or without Rspondin3 i.v. (Data are representative of three independent experiments with five mice per group). Graphs show the mean \pm s.d, with each dot representing an individual mouse. Statistical significance was determined by two-way ANOVA with Sidak's multiple comparisons test using GraphPad Prism with individual $P$ values (left to right) are: $n s P>0.9999, n s P=0.9301$, ns $P>0.9999$, ${ }^{\star \star \star \star ~} P<0.0001, n s P=0.9992, n s P>0.9999$, ns $P>0.9999$, ns $P>0.9999$, ns $P>0.9999$. 
a

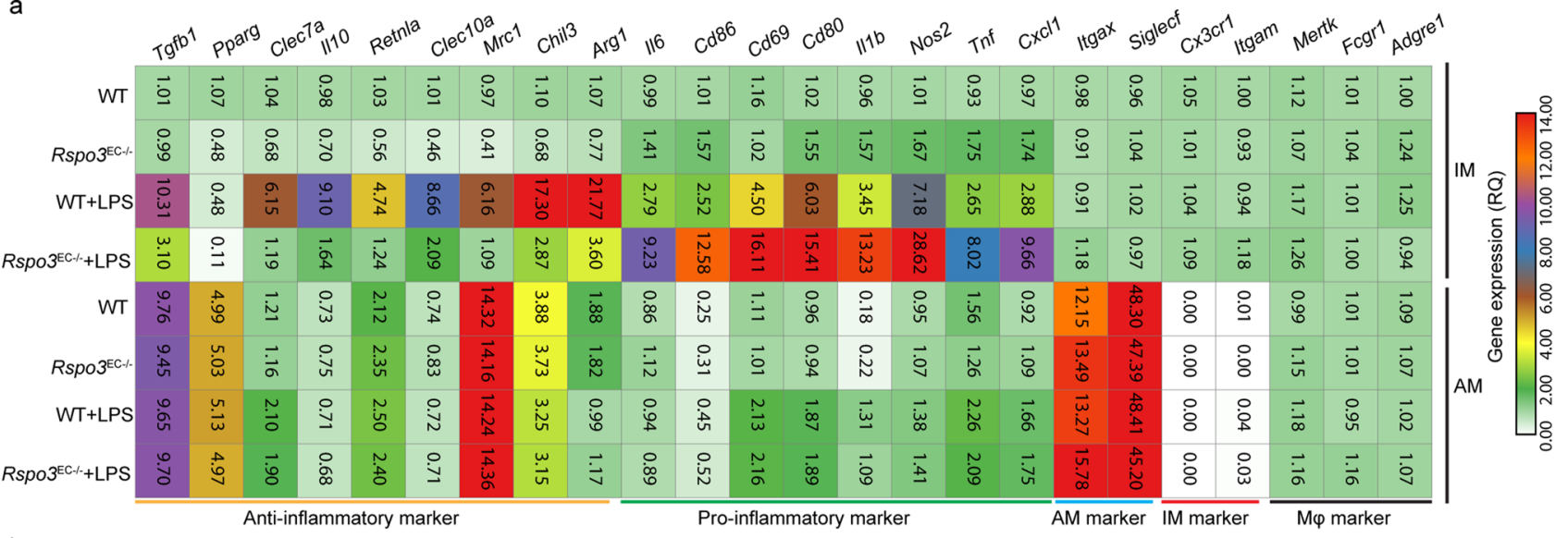

b

WT
Rspo3
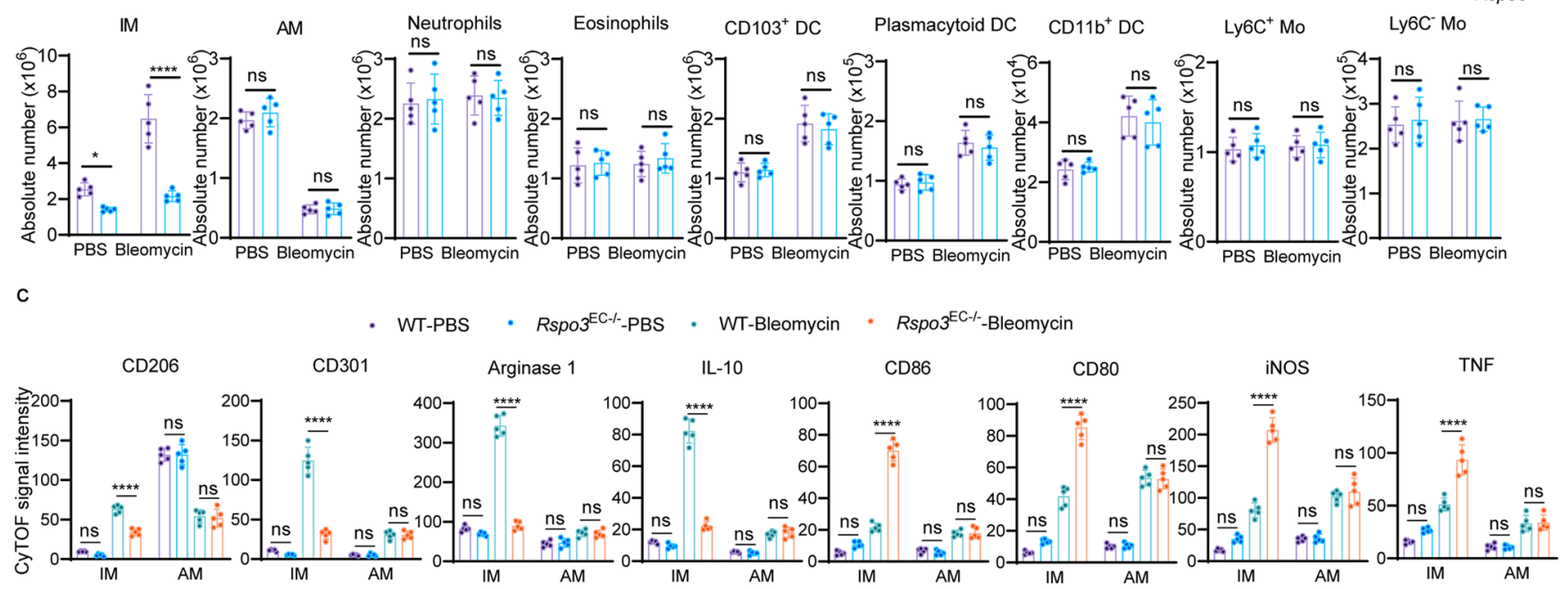

d
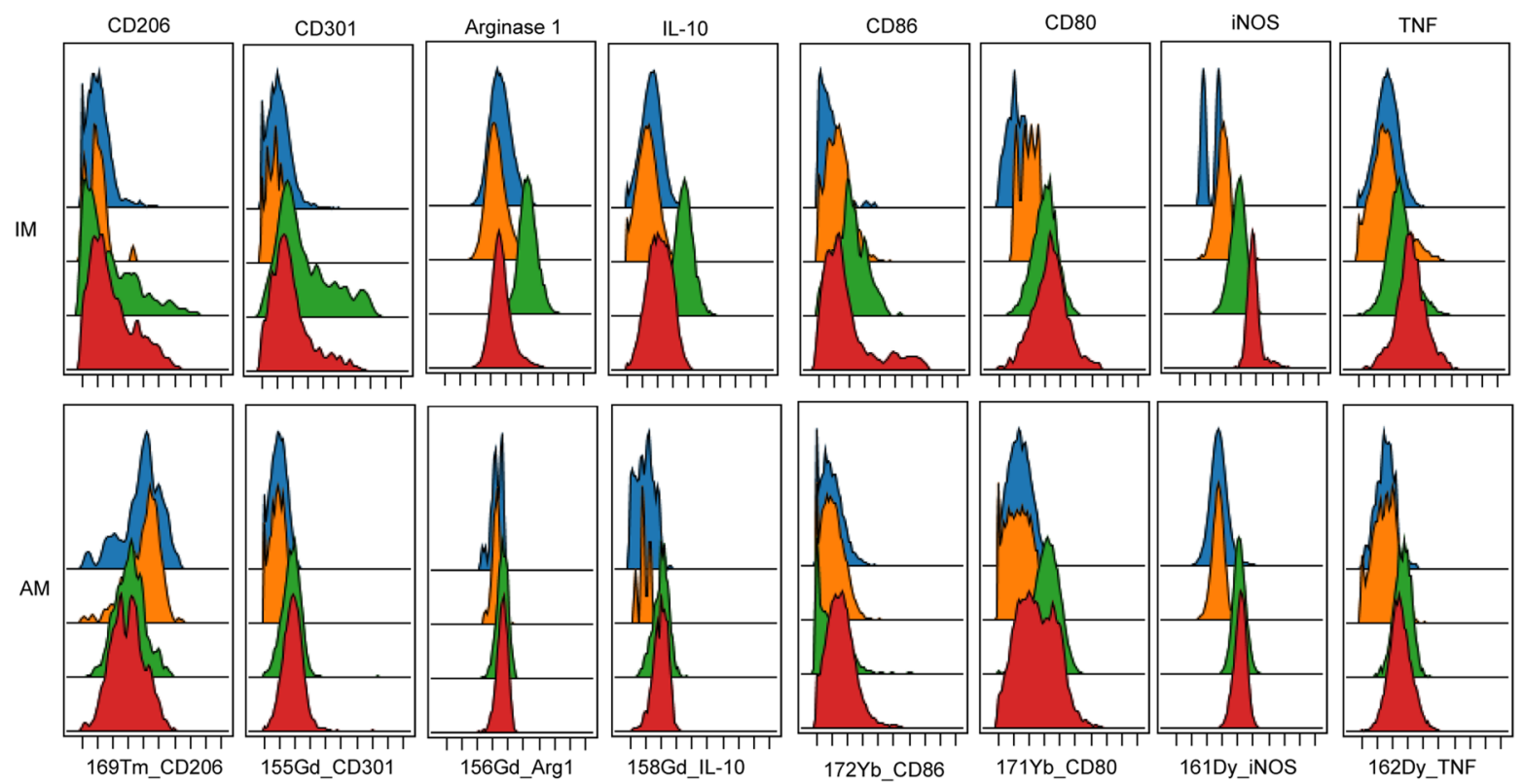

WT/PBS

Rspo3 $3^{E C-\mu / P B S}$

WT/Bleomycin

Rspo3 ${ }^{E C-r / B l}$ Bleomycin

\section{Extended Data Fig. 4 | See next page for caption.}


Extended Data Fig. 4 | Rspondin3 regulates lung interstitial macrophage phenotype transition following acute lung injury. a, Gene expression profiling performed by qPCR to evaluate the expression of macrophage marker genes, anti-inflammatory and pro-inflammatory genes in sorted IM and AM from WT and Rspo $3^{\mathrm{EC}-}$ mice lungs under baseline conditions and endotoxemia. Heatmap shows the fold change of gene levels as normalized to WT IM at basal conditions (Data are representative of three independent experiments, $n=3$ samples per group); $\mathbf{b}$, Lung myeloid populations analyzed by CyTOF in WT and $R$ spo3 ${ }^{\mathrm{EC}-\mathrm{-}}$ mice subjected to bleomycin induced acute lung injury and basal conditions (Data are representative of three independent experiments with five mice per group). Graphs show the mean \pm s.d, with each dot representing an individual mouse. Statistical significance was determined by two-way ANOVA with Sidak's multiple comparisons test using GraphPad Prism with individual $P$ values (left to right) are: IM ( ${ }^{\star} P=0.0487$, $\left.{ }^{\star \star \star \star} P<0.0001\right)$, AM (ns $P<0.3554$, ns $P<0.9932$ ), neutrophils (ns $P=0.9329$, ns $P=0.9798$ ), eosinophils (ns $P=0.9557$, ns $P=0.7925$ ), Ly6C + Mo (ns $P=0.8486$, ns $P=0.9777$ ), Ly6C- Mo (ns $P=0.9040$, ns $P=0.9863$ ), $C D 103^{+} D C$ (ns $P=0.9526$, ns $P=0.7602$ ), plasmacytoid $D C$ ( $n s P=0.9410, n s P=0.7104$ ), $C D 11 b^{+}$ DC (ns $P=0.9613$, ns $P=0.7995)$; c, Levels of the anti-inflammatory markers (CD206, CD301, arginase 1, IL-10) and pro-inflammatory markers (CD86, CD80, iNOS, TNF) in IM in WT and Rspo3 ${ }^{E C-\%}$ mice under baseline conditions and post bleomycin injury for 5 days as measured by CyTOF (Data are representative of three independent experiments with $n=5$ mice per group). Graphs show the mean \pm s.d, with each dot representing an individual mouse. Statistical significance was determined by two-way ANOVA with Tukey's multiple comparisons test using GraphPad Prism with individual $P$ values (left to right) are: $C D 206$ (ns $P=0.7843$, ${ }^{\star \star \star *} P<0.0001$, ns $P>0.9999$, ns $P=0.9642$ ), CD301 (ns $P=0.5538$, ${ }^{\star \star \star \star} P<0.0001$, ns $P>0.9999$, ns $P>0.9999$ ), arginase

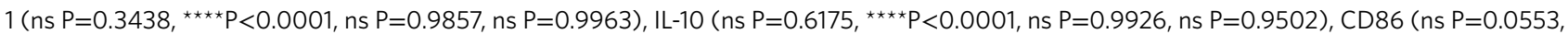

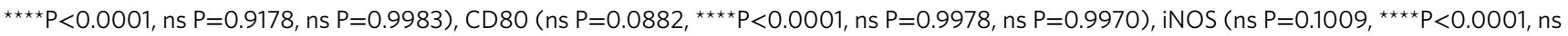
$\mathrm{P}=0.9991$, ns $\mathrm{P}=0.7604$ ), TNF (ns $\mathrm{P}=0.0736$, $\star \star \star \star ~ P<0.0001$, ns $\mathrm{P}=0.9997$, ns $\mathrm{P}=0.9979$ ); $\mathbf{d}$, Representative overlaid CyTOF histograms showing the CyTOF signal intensity of the anti-inflammatory markers (CD206, CD301, arginase 1, IL-10) and pro-inflammatory markers (CD86, CD80, TNF, iNOS) in IM and AM in WT and Rspo3 ${ }^{\mathrm{EC} /-}$ mice at baseline conditions or 5 days after bleomycin i.t. for data in (e). 
a
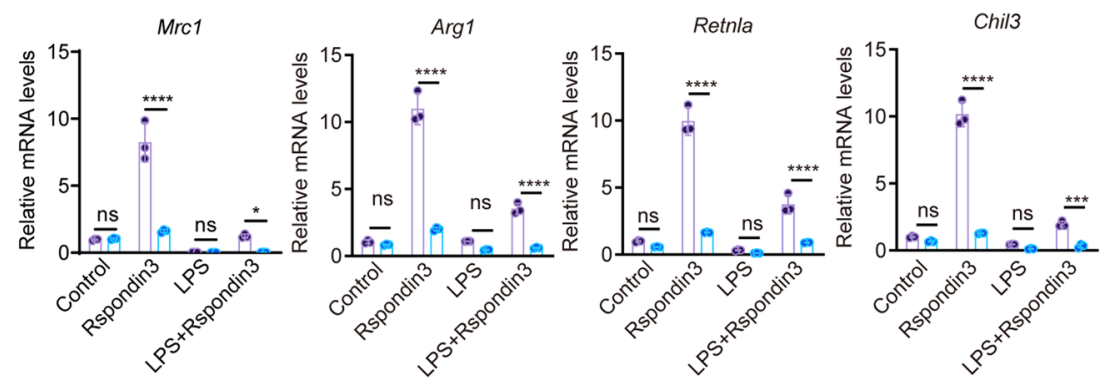

- siNC

Cd86

$111 b$
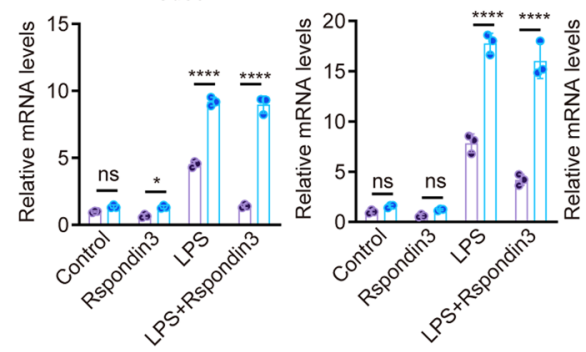

Tnf

Cxcl1

b

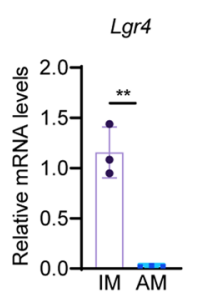

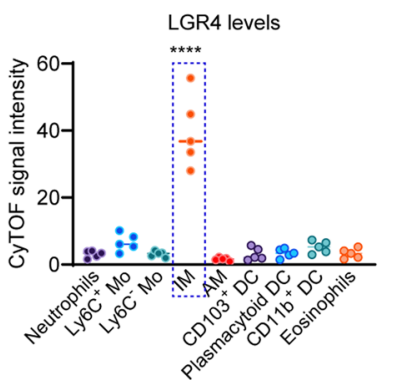

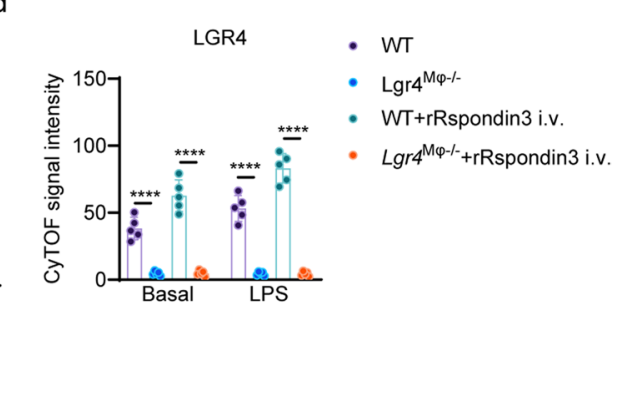

Extended Data Fig. 5 | Macrophage expressed receptor LGR4 is required for Rspondin3-induced macrophage phenotype transition. a, Gene expression levels of anti-inflammatory marker genes (Mrc1, Arg1, Retnla, Chil3) and pro-inflammatory marker genes (Cd86, II16, Tnf, CxC17) measured by qPCR in BMDMs with or without Lgr4 depletion at basal conditions, or treated with Rspondin3, LPS alone or combination of both for $24 \mathrm{~h}$ from three independent experiments with $n=3$ samples per group (mean $\pm \mathrm{sd}$ ); Statistical significance was determined by two-way ANOVA with Sidak's multiple comparisons test using GraphPad Prism with individual $P$ values (left to right) are: $\operatorname{Mrcl}$ ( $\mathrm{ns} P=0.9997,{ }^{\star \star \star \star *} \mathrm{P}<0.0001, \mathrm{~ns} \mathrm{P}>0.9999,{ }^{*} \mathrm{P}=0.0488$ ), Arg1 (ns $\mathrm{P}=0.9708$, ${ }^{\star \star \star \star \star} \mathrm{P}<0.0001$, ns $\mathrm{P}=0.3532$, ${ }^{\star \star \star \star} \mathrm{P}<0.0001$ ), Retnla (ns $\mathrm{P}=0.7211$, ${ }^{\star \star \star \star} \mathrm{P}<0.0001$, ns $\mathrm{P}=0.9663$, ${ }^{\star \star \star \star} \mathrm{P}<0.0001$ ), Chil3 (ns $\mathrm{P}=0.6858$, ${ }^{\star \star \star \star} \mathrm{P}<0.0001$, ns $\mathrm{P}=0.6939$, ${ }^{\star \star \star} \mathrm{P}=0.0001$ ), $\mathrm{Cd} 86$ (ns $\mathrm{P}=0.3993$, ${ }^{\star} \mathrm{P}=0.0419$, ${ }^{\star \star \star \star} \mathrm{P}<0.0001,{ }^{\star \star \star \star \star} \mathrm{P}<0.0001$ ), Il1b (ns $\mathrm{P}=0.8936$, ns $\mathrm{P}=0.8526$, ${ }^{\star \star \star \star *} \mathrm{P}<0.0001$, ${ }^{\star \star \star \star \star} P<0.0001$ ), $\operatorname{Tnf}$ (ns $P>0.9999$, ns $P=0.3417$, ${ }^{\star \star \star \star} P<0.0001$, $\left.{ }^{\star \star \star \star} P<0.0001\right), C x c 17$ (ns $P=0.9976, n s P=0.9584,{ }^{\star \star \star \star} P<0.0001,{ }^{\star \star \star \star \star} P<0.0001$ ). b, mRNA levels of $L g r 4$ in sorted IM and AM measured by $q P C R ; n=3$ samples per group (mean $\pm s d$ ), two-sided unpaired t-test was determined using GraphPad Prism. ${ }^{\star \star} P=0.0012$. c, Levels of LGR4 in myeloid populations in mice lung evaluated by CyTOF (Data are representative of three independent experiments with $n=5$ mice per group). Graphs show the mean \pm s.d, with each dot representing an individual mouse. Statistical significance was determined by ordinary one-way ANOVA with Dunnett's multiple comparisons test using GraphPad Prism. ${ }^{\star \star \star \star P}<<0.0001$. d, Levels of LGR4 in IM in WT and Lgr4 ${ }^{M \varphi-1}$ mice with or without Rspondin3 i.v. under baseline conditions and post sublethal LPS challenge for 24h measured by CyTOF (Data are representative of three independent experiments with $n=5$ mice per group). Graphs show the mean $\pm s$. $d$, with each dot representing an individual mouse. Statistical significance was determined by two-way ANOVA with Tukey's multiple comparisons test using GraphPad Prism with individual P values (left to right) are: ${ }^{\star \star \star \star} \mathrm{P}<0.0001,{ }^{\star \star \star \star} \mathrm{P}<0.0001,{ }^{\star \star \star \star} \mathrm{P}<0.0001,{ }^{\star \star \star \star} \mathrm{P}<0.0001$. 

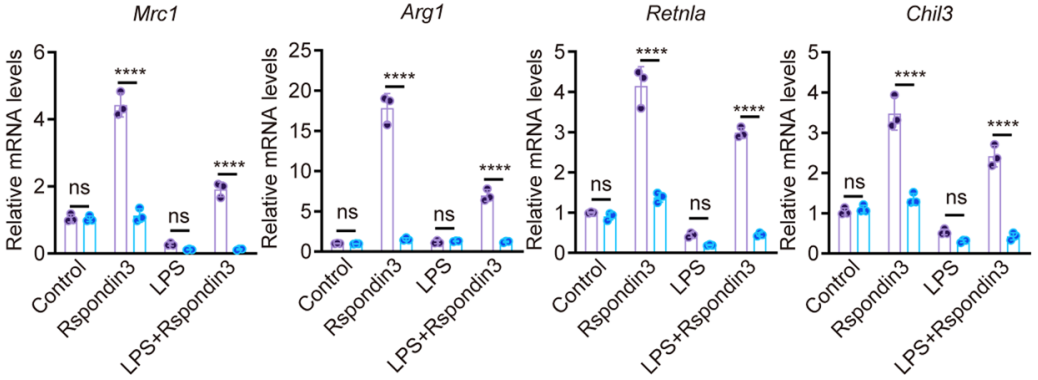

- WT

Cd86

$111 b$

Tnf

$\mathrm{CxCl1}$
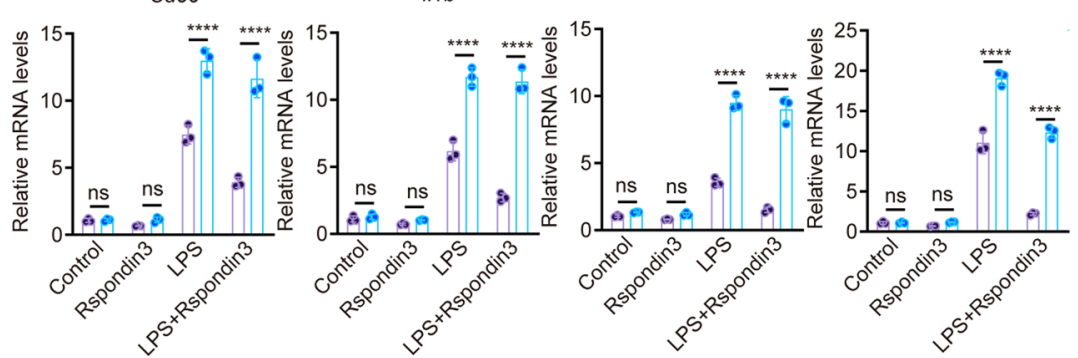

Extended Data Fig. 6 | $\boldsymbol{\beta}$-catenin is required for Rspondin3-induced macrophage phenotype transition. Levels of anti-inflammatory marker genes

(Mrc1, Arg1, Retnla, Chil3) and pro-inflammatory marker genes (Cd86, II1b, Tnf, Cxcl1) measured by qPCR in BMDMs from Ctnnb1 ${ }^{\text {M } \omega-1-}$ and WT mice at basal condition, or treated with Rspondin3, LPS alone or combination of both for 24h; Data are representative of three independent experiments with $\mathrm{n}=3$ samples per group (mean $\pm \mathrm{sd}$ ); Statistical significance was determined by two-way ANOVA with Tukey's multiple comparisons test using GraphPad Prism with individual $P$ values (left to right) are: $\operatorname{Mrc1}$ (ns $\left.P>0.9999,{ }^{\star \star \star \star} P<0.0001, n s P>0.9999,{ }^{\star \star \star \star *} P<0.0001\right), \operatorname{Arg} 1$ (ns $P>0.9999,{ }^{\star \star \star \star \star} P<0.0001$, ns $P>0.9999$, ${ }^{\star \star \star \star} P<0.0001$ ), Retnla (ns $P>0.9999$, ${ }^{\star \star \star \star} P<<0.0001$, ns $P=0.9716$, ${ }^{\star \star \star \star} P<0.0001$ ), Chil3 (ns $P=0.9998$, ${ }^{\star \star \star \star \star} P<0.0001, n s P=0.8135$,

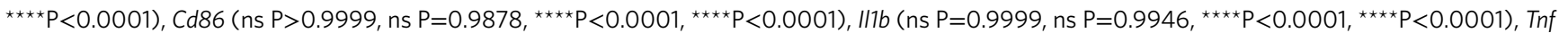
(ns $P=0.9854$, ns $P=0.9426$, ${ }^{\star \star \star \star} P<0.0001$, ${ }^{\star \star \star \star P}<0.0001$ ), Cxcl1 (ns $P>0.9999$, ns $P=0.9975,{ }^{\star \star \star \star} P<0.0001,{ }^{\star \star \star \star ~} P<0.0001$ ). 


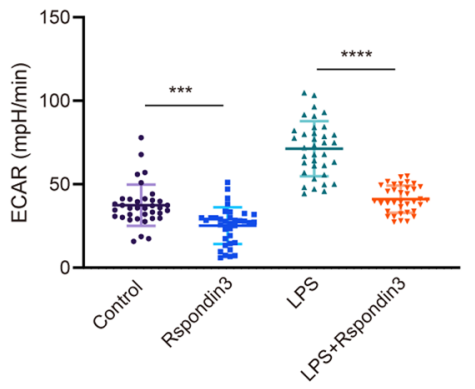

d

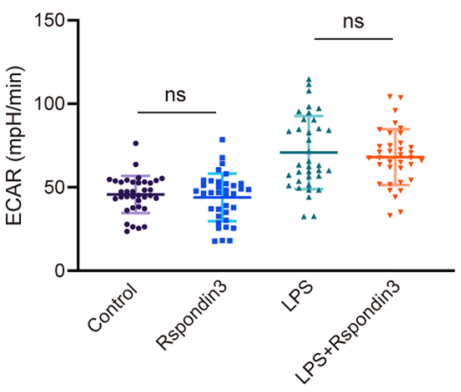

f

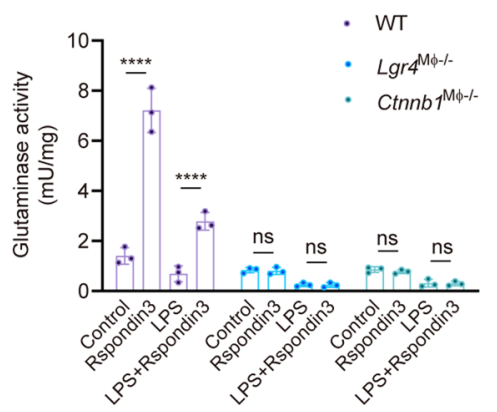

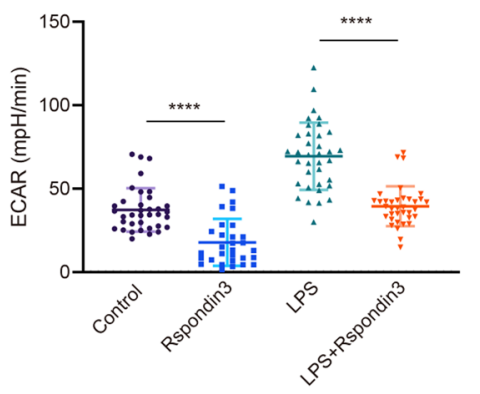

e

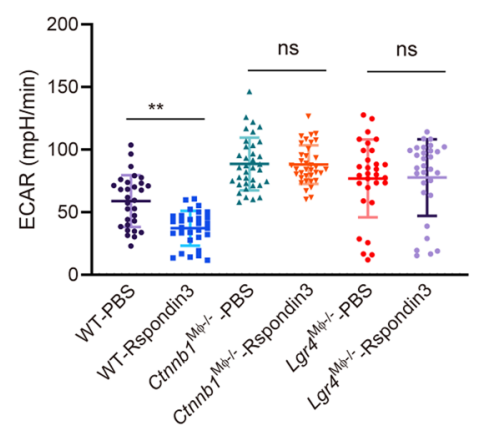

g

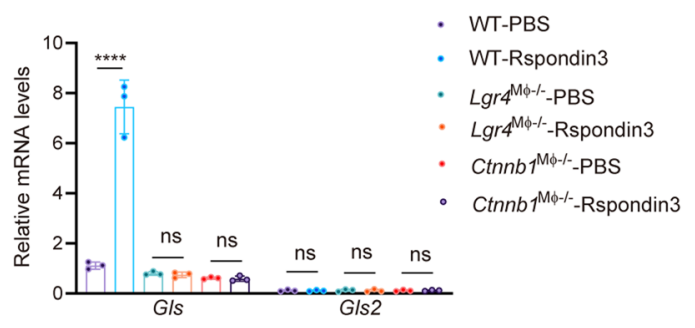

Extended Data Fig. 7 | Rspondin3 mediated increase in mitochondrial respiration through glutamine metabolism. a, Basal extracellular acidification rate (ECAR) measured in macrophages stimulated with Rspondin3, LPS alone or in combination for $24 \mathrm{~h}$ with complete DMEM medium containing $10 \mathrm{mM}$ glucose, $2 \mathrm{mM}$ glutamine, $2 \mathrm{mM}$ sodium pyruvate as substrate; Data are representative of three independent experiments, with 10-12 samples per group. Graphs show the mean \pm s.d, with each dot representing an individual sample per time point. Statistical significance was determined by ordinary one-way ANOVA with Tukey's multiple comparisons test using GraphPad Prism, P values are ${ }^{\star \star \star P}=0.0003,{ }^{\star \star \star \star P} P<0.0001$. b, Basal ECAR measured in macrophages stimulated with Rspondin3, LPS alone or in combination with only $2 \mathrm{mM}$ glutamine as substrate; Data are representative of three independent experiments, with 10-12 samples per group. Graphs show the mean \pm s.d, with each dot representing an individual sample per time point. Statistical significance was determined by ordinary one-way ANOVA with Tukey's multiple comparisons test using GraphPad Prism, P values are ${ }^{\star \star \star \star} \mathrm{P}<0.0001,{ }^{\star \star \star \star} \mathrm{P}<0.0001$. c, Basal ECAR measured in BMDMs stimulated with Rspondin3, LPS alone or in combination with only $10 \mathrm{nM}$ free fatty acid as palmitate bound to BSA added as substrate; Data are representative of three independent experiments, with 10-12 samples per group. Graphs show the mean \pm s.d, with each dot representing an individual sample per time point. Statistical significance was determined by ordinary one-way ANOVA with Tukey's multiple comparisons test using GraphPad Prism, $P$ values are ns $P=0.9681, n s P=0.3389$. d, Basal ECAR measured in BMDMs stimulated with Rspondin3, LPS alone or in combination with only10 mM glucose as substrate; Data are representative of three independent experiments, with 10-12 samples per group. Graphs show the mean \pm s.d, with each dot representing an individual sample per time point. Statistical significance was determined by ordinary one-way ANOVA with Tukey's multiple comparisons test using GraphPad Prism, P values are ns P=0.9694, ns P=0.9000. e, Basal

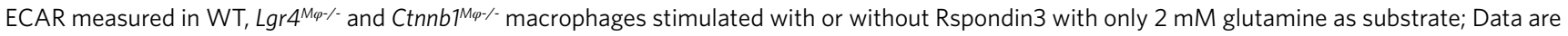
representative of three independent experiments, graphs show the mean \pm s.d, with each dot representing an individual sample per time point. Statistical significance was determined by ordinary one-way ANOVA with Tukey's multiple comparisons test using GraphPad Prism, $P$ values are ${ }^{\star \star} P=0.0041, n s$ $\mathrm{P}>0.9999$, ns P>0.9999. f, Glutaminase activity measured in WT, Lgr4 ${ }^{M \omega-} /-$ and Ctnnb ${ }^{\mathrm{M} \varphi-/}$ BMDMs under basal, or stimulated with Rspondin3, LPS alone or in combination. Data are representative of three independent experiments (mean $\pm \mathrm{sd}$ ); Statistical significance was determined by two-way ANOVA with Tukey's multiple comparisons test using GraphPad Prism. $P$ values (left to right) are ${ }^{\star \star \star \star} P<0.0001,{ }^{\star \star \star \star} P<0.0001, n s P=0.9995, n s P>0.9999$, ns $\mathrm{P}=0.9881$, ns $\mathrm{P}>0.9999$. , Relative gene expression of glutaminase genes - Gls and Gls2 in WT, Lgr4 ${ }^{\mathrm{M} \varphi-/}$ and Ctnnb $7^{\mathrm{M} \varphi-/}$ BMDMs with or without Rspondin3 stimulation measured by qPCR; Data are representative of three independent experiments, $\mathrm{n}=3$ samples from three individual mice per group (mean $\pm \mathrm{sd}$ ), statistical significance was determined by two-way ANOVA with Tukey's multiple comparisons test using GraphPad Prism. P values are ns $\mathrm{P}>0.9999,{ }^{\star \star \star \star} \mathrm{P}<0.0001$. 
a

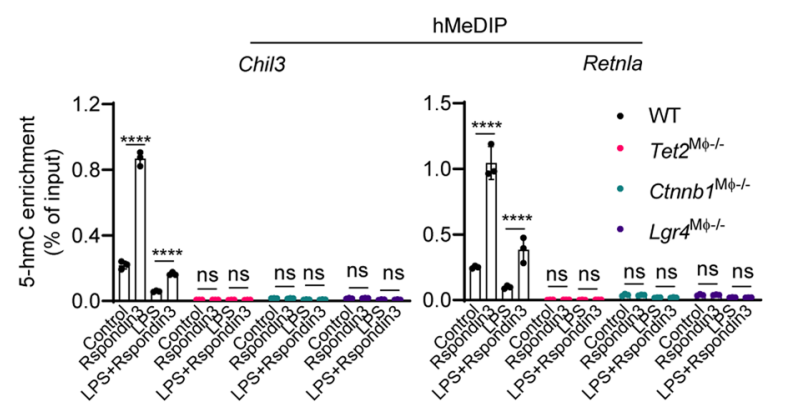

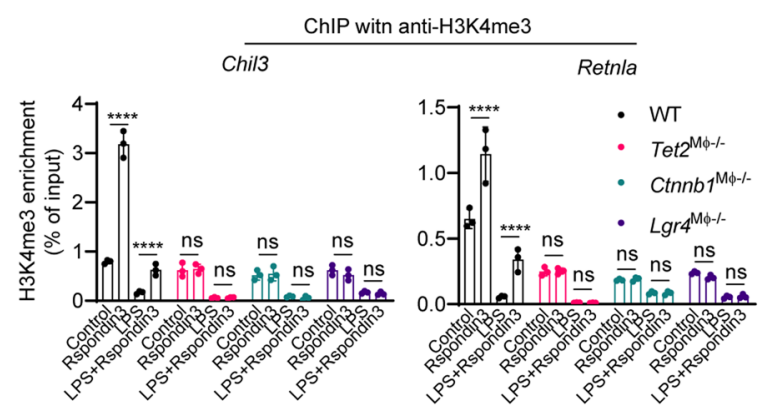

C
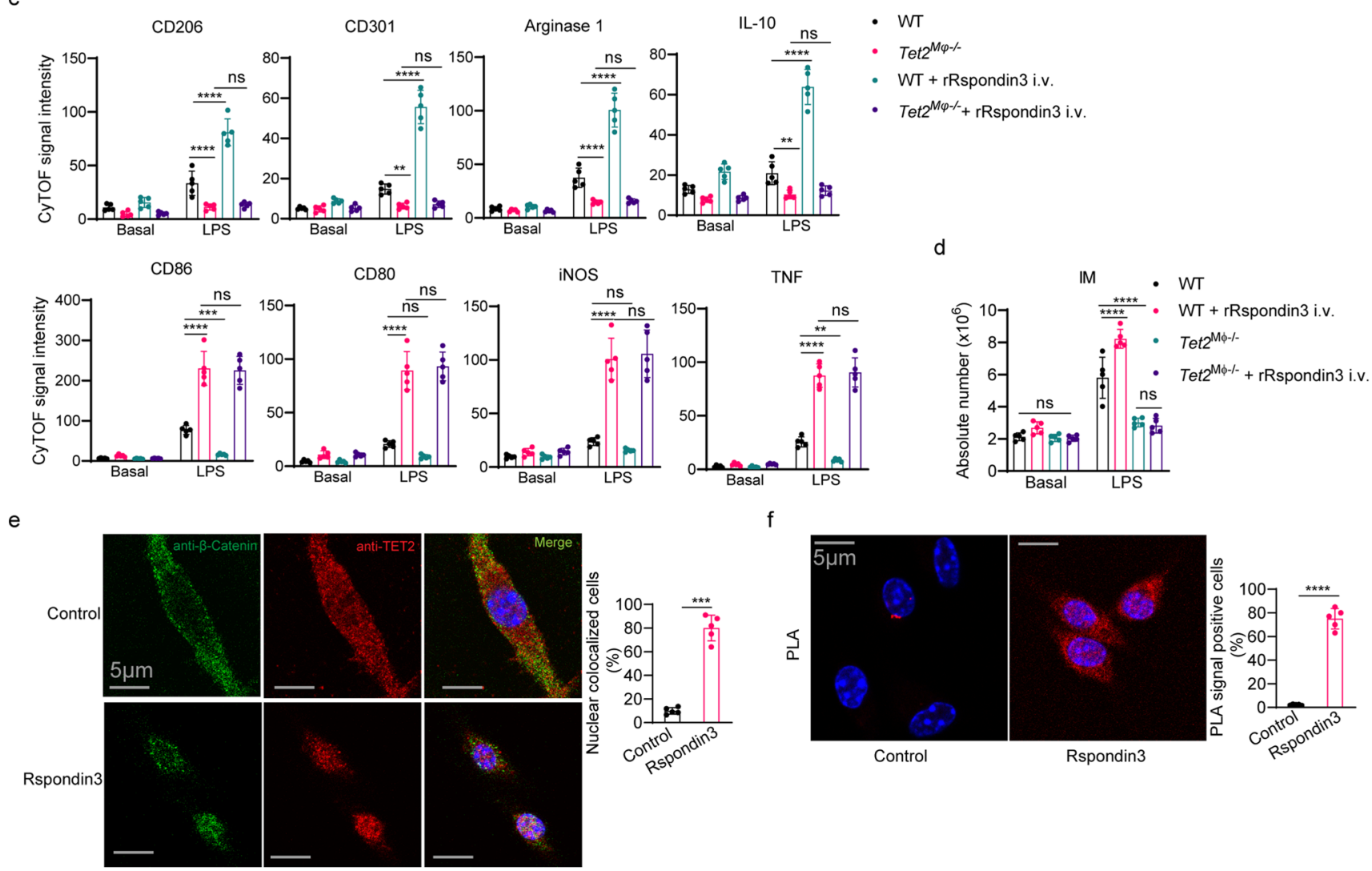

f

g

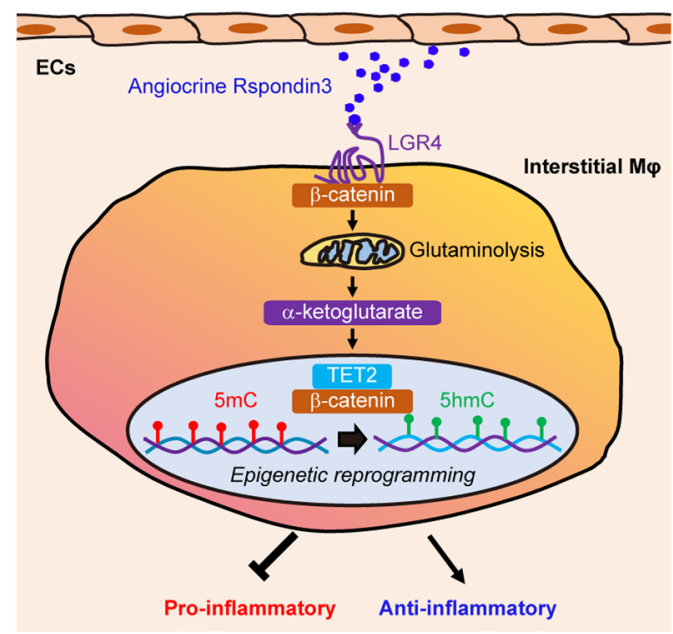

Extended Data Fig. 8 | See next page for caption. 
Extended Data Fig. 8 | Rspondin3 induces TET2-mediated DNA hydroxymethylation of anti-inflammatory genes. a, hMeDIP performed on WT, Lgr4 ${ }^{\mathrm{M} \varphi-1}$, Ctnnb $7^{\mathrm{M} \varphi-/}$ and Tet2 ${ }^{\mathrm{M} \varphi-/}$ BMDMs treated with Rspondin3, LPS alone or combination of both, and qPCR with primers targeting the proximal promoters of the indicated genes were used to detect the enrichment of $5 \mathrm{hmC}$; Data are representative of three independent experiments, $\mathrm{n}=3$ samples per group (mean $\pm \mathrm{sd}$ ); Statistical significance was determined by two-way ANOVA with Tukey's multiple comparisons test using GraphPad Prism. $P$ values (left to right) are: Chil3 ( ${ }^{\star \star \star \star} P<0.0001$, ${ }^{\star \star \star \star} P<0.0001$, ns $P>0.9999$, ns $P>0.9999$, ns $P=0.9998$, ns $P>0.9999, n s P=0.9983$, ns $P>0.9999$ ), Retnla ( ${ }^{\star \star \star *} \mathrm{P}<0.0001$, ${ }^{\star \star \star \star} \mathrm{P}<0.0001$, ns $\mathrm{P}>0.9999$, ns $\mathrm{P}>0.9999, \mathrm{P}>0.9999$, ns $\mathrm{P}>0.9999$, ns $\mathrm{P}>0.9999$, ns $\left.\mathrm{P}>0.9999\right)$. b, ChIP with an anti-H3K4me3

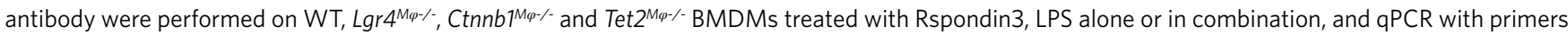
targeting the proximal promoters of the indicated genes were used to detect the enrichment of H3K4me3; Data are representative of three independent experiments, $n=3$ samples per group (mean $\pm \mathrm{sd}$ ); Statistical significance was determined by two-way ANOVA with Tukey's multiple comparisons test using GraphPad Prism. $P$ values (left to right) are: Chil3 ( ${ }^{\star \star \star \star} P<0.0001$, ${ }^{\star \star \star \star} P<0.0001$, ns $P=0.9920$, ns $P>0.9999, n s P=0.9883$, ns $P=0.9843$, ns $P=0.6545$, ns $P>0.9900$ ), Retnla ( ${ }^{\star \star \star \star} P<0.0001$, ${ }^{\star \star \star \star} P<0.0001$, ns $P=0.9998$, ns $P>0.9999, P=0.9992$, ns $P>0.9999$, ns $P=0.8940$, ns $P=0.9994$ ).

c, Bar figure showing levels of anti-inflammatory markers and pro-inflammatory markers in lung IM in WT and Tet2 ${ }^{\mathrm{M} \varphi-1-}$ mice with or without Rspondin3 i.v. under basal and post-sublethal LPS challenge for $24 \mathrm{~h}$ as measured by CyTOF (data are representative of three independent experiments with five mice per group). Graphs show the mean \pm s.d, with each dot representing an individual mouse. Statistical significance was determined by two-way ANOVA with Tukey's multiple comparisons test using GraphPad Prism with individual $P$ values (left to right) are: $C D 206\left({ }^{\star \star \star \star} P<0.0001,{ }^{\star \star \star \star} P<0.0001\right.$, ns $P=0.9618$ ),

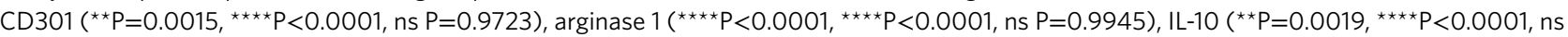

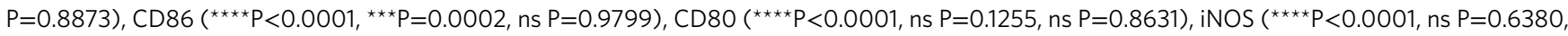

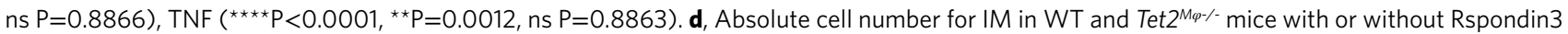
i.v. under basal conditions and post sublethal LPS challenge for $24 \mathrm{~h}$ measured by CyTOF (Data are representative of three independent experiments with five mice per group). Graphs show the mean \pm s.d., with each dot representing an individual mouse. Statistical significance was determined by two-way ANOVA with Tukey's multiple comparisons test using GraphPad Prism with individual P values (left to right) are $n s P>0.4182$, ${ }^{\star \star \star \star *} P<0.0001$, ${ }^{* * \star *} \mathrm{P}<0.0001$, ns $\mathrm{P}=0.9444$; e, Representative confocal images for immunofluorescent staining with anti-TET2 and anti- $\beta$-catenin antibodies performed on BMDMs stimulated with Rspondin3 or PBS from three independent repeats, and quantified data are shown (right) with $n=5$ samples per group (mean \pm sd); Statistical significance was determined by two-tailed unpaired t test using GraphPad Prism. ${ }^{\star \star \star \star} P<0.0001$. f, Representative confocal images for in situ proximity ligation assay (PLA) with anti-TET2 and anti- $\beta$-catenin antibodies to detect interactions were performed on BMDMs stimulated with Rspondin3 or PBS (three independent repeats), and quantified data are shown (right) with $n=5$ samples per group (mean $\pm s d$ ); Statistical significance was determined by two-tailed unpaired $t$ test using GraphPad Prism. ${ }^{\star \star \star \star} \mathrm{P}<0.0001$. g, Model: The angiocrine-metabolic-epigenetic axis regulates lung IM phenotypic transition. Lung ECs release Rspondin3, which binds to its cell surface receptor-LGR4 in IM and activates $\beta$-catenin leading to increased $\alpha$-ketoglutarate concentration through activation of glutaminolysis, followed by induction of TET2 mediated epigenetic reprograming. TET2 mediated DNA hydroxymethylation increases expression of anti-inflammatory genes in IM and prevents inflammatory lung injury. 


\section{Reporting Summary}

Nature Research wishes to improve the reproducibility of the work that we publish. This form provides structure for consistency and transparency in reporting. For further information on Nature Research policies, see Authors \& Referees and the Editorial Policy Checklist.

\section{Statistics}

For all statistical analyses, confirm that the following items are present in the figure legend, table legend, main text, or Methods section.

$\mathrm{n} / \mathrm{a}$ Confirmed

$\bigotimes$ The exact sample size $(n)$ for each experimental group/condition, given as a discrete number and unit of measurement

\A statement on whether measurements were taken from distinct samples or whether the same sample was measured repeatedly

The statistical test(s) used AND whether they are one- or two-sided

$\triangle$ Only common tests should be described solely by name; describe more complex techniques in the Methods section.

X A description of all covariates tested

\A description of any assumptions or corrections, such as tests of normality and adjustment for multiple comparisons

A full description of the statistical parameters including central tendency (e.g. means) or other basic estimates (e.g. regression coefficient)

AND variation (e.g. standard deviation) or associated estimates of uncertainty (e.g. confidence intervals)

Х

For null hypothesis testing, the test statistic (e.g. $F, t, r$ ) with confidence intervals, effect sizes, degrees of freedom and $P$ value noted Give $P$ values as exact values whenever suitable.

Х For Bayesian analysis, information on the choice of priors and Markov chain Monte Carlo settings

Х $\square$ For hierarchical and complex designs, identification of the appropriate level for tests and full reporting of outcomes

Х $\square$ Estimates of effect sizes (e.g. Cohen's $d$, Pearson's $r$ ), indicating how they were calculated

Our web collection on statistics for biologists contains articles on many of the points above.

\section{Software and code}

Policy information about availability of computer code

Data collection

All the flow cytometry data were acquired on a CytoFLEX S Flow Cytometer (Beckman).

Seahorse XF96 Extracellular Flx Analyzer (Agilent) was used for determining oxygen consumption.

ELISA data were collected on a SynergyTM HTX multi-mode microplate reader (BioTek).

Data for TET activities, JMJD3/UTX activities, $\alpha$-ketoglutarate and succinate levels, $5 \mathrm{hmC}$ levels, and glutaminase activities were collected on a SynergyTM HTX multi-mode microplate reader (BioTek) via biochemical assays.

All the qPCR data for RNA levels, ChIP, and hMeDIP were collected on an ABI Prism 7000 system.

All the microscopic pictures were acquired with Zeiss Laser Scanning Confocal Microscope (LSM) with Zen software 3.1.

Cytometry by time-of-flight mass spectrometry (CyTOF) were performed with a Helios CyTOF Mass cytometer (FLUIDIGM Inc, USA).

Data analysis

Kaluza Analysis 2.1 software (Beckman) was used for analysis of flow cytometry data.

Wave software 2.6.1 (Agilent) was used for analysis of Seahorse data.

ELISA data were analyzed using Gen 5 software (BioTek).

Data for TET activities, JMJD3/UTX activities, $\alpha$-ketoglutarate and succinate levels, $5 \mathrm{hmC}$ levels, and glutaminase activities were analyzed with Gen 5 software (basic version).

qPCR data for RNA levels, ChIP, and hMeDIP were analyzed and exported by the QuantStudio software v1.3.

All the microscopic pictures were analyzed by ImageJ software v1.52p.

Final graphs were made in GraphPad prism v8.0, and figures were arranged and edited in Adobe Illustrator2019.

Schematics were made by Microsoft PowerPoint.

CyTOF data were analyzed using Cytobank (https://www.cytobank.org/).

Heatmap were generized using morpheus (https://software.broadinstitute.org/morpheus/). 
Policy information about availability of data

All manuscripts must include a data availability statement. This statement should provide the following information, where applicable:

- Accession codes, unique identifiers, or web links for publicly available datasets

- A list of figures that have associated raw data

- A description of any restrictions on data availability

Source data are provided with this paper. Other data that support the findings of this study are available from the corresponding author upon request.

\section{Field-specific reporting}

Please select the one below that is the best fit for your research. If you are not sure, read the appropriate sections before making your selection.

\ Life sciences

Behavioural \& social sciences

Ecological, evolutionary \& environmental sciences

For a reference copy of the document with all sections, see nature.com/documents/nr-reporting-summary-flat.pdf

\section{Life sciences study design}

All studies must disclose on these points even when the disclosure is negative.

Sample size

We did not use statistical method to determine sample size; We chose sample size based on our prior experience as for in vivo and in vitro experiments, the sample size for in vivo study was at least five mice per group and the data was routinely collected across at least three independent experiments which turned out similar results; for in vitro study, the sample size was at least three independent samples with three independent repeats showed similar results.

Data exclusions no samples or animals were excluded from the analysis.

Replication Experiments were independently repeated three times which turned out similar results to ensure the reproducibility of the findings.

Randomization Littermates were always used as controls. In comparisons between different mouse strains, mice were segregated based on their genotypes. Mice from the same genotype were randomly ascribed to different comparison groups.

Blinding Investigators were blinded to the genotype of the mice during sample preparation and data collection. And investigators were blinded to the group allocation during data analysis.

\section{Behavioural \& social sciences study design}

All studies must disclose on these points even when the disclosure is negative.

Study description

Research sample

Sampling strategy

Data collection

Timing

Data exclusions

Non-participation

Randomization
Briefly describe the study type including whether data are quantitative, qualitative, or mixed-methods (e.g. qualitative cross-sectional, quantitative experimental, mixed-methods case study).

State the research sample (e.g. Harvard university undergraduates, villagers in rural India) and provide relevant demographic information (e.g. age, sex) and indicate whether the sample is representative. Provide a rationale for the study sample chosen. For studies involving existing datasets, please describe the dataset and source.

Describe the sampling procedure (e.g. random, snowball, stratified, convenience). Describe the statistical methods that were used to predetermine sample size $O R$ if no sample-size calculation was performed, describe how sample sizes were chosen and provide a rationale for why these sample sizes are sufficient. For qualitative data, please indicate whether data saturation was considered, and what criteria were used to decide that no further sampling was needed.

Provide details about the data collection procedure, including the instruments or devices used to record the data (e.g. pen and paper, computer, eye tracker, video or audio equipment) whether anyone was present besides the participant(s) and the researcher, and whether the researcher was blind to experimental condition and/or the study hypothesis during data collection.

Indicate the start and stop dates of data collection. If there is a gap between collection periods, state the dates for each sample cohort.

If no data were excluded from the analyses, state so OR if data were excluded, provide the exact number of exclusions and the rationale behind them, indicating whether exclusion criteria were pre-established.

State how many participants dropped out/declined participation and the reason(s) given OR provide response rate OR state that no participants dropped out/declined participation.

If participants were not allocated into experimental groups, state so OR describe how participants were allocated to groups, and if allocation was not random, describe how covariates were controlled. 


\section{Ecological, evolutionary \& environmental sciences study design}

All studies must disclose on these points even when the disclosure is negative.

$\begin{array}{ll}\text { Study description } & \begin{array}{l}\text { Briefly describe the study. For quantitative data include treatment factors and interactions, design structure (e.g. factorial, nested, } \\ \text { hierarchical), nature and number of experimental units and replicates. }\end{array}\end{array}$

Research sample Describe the research sample (e.g. a group of tagged Passer domesticus, all Stenocereus thurberi within Organ Pipe Cactus National Monument), and provide a rationale for the sample choice. When relevant, describe the organism taxa, source, sex, age range and any manipulations. State what population the sample is meant to represent when applicable. For studies involving existing datasets, describe the data and its source.

Sampling strategy

Note the sampling procedure. Describe the statistical methods that were used to predetermine sample size OR if no sample-size calculation was performed, describe how sample sizes were chosen and provide a rationale for why these sample sizes are sufficient.

Data collection

Describe the data collection procedure, including who recorded the data and how.

Timing and spatial scale

Indicate the start and stop dates of data collection, noting the frequency and periodicity of sampling and providing a rationale for these choices. If there is a gap between collection periods, state the dates for each sample cohort. Specify the spatial scale from which the data are taken

Data exclusions

If no data were excluded from the analyses, state so OR if data were excluded, describe the exclusions and the rationale behind them, indicating whether exclusion criteria were pre-established.

Reproducibility

Describe the measures taken to verify the reproducibility of experimental findings. For each experiment, note whether any attempts to repeat the experiment failed OR state that all attempts to repeat the experiment were successful.

Randomization

Describe how samples/organisms/participants were allocated into groups. If allocation was not random, describe how covariates were controlled. If this is not relevant to your study, explain why.

Blinding

Describe the extent of blinding used during data acquisition and analysis. If blinding was not possible, describe why OR explain why blinding was not relevant to your study.

Did the study involve field work? $\square$ Yes $\square$ No

Field work, collection and transport

Field conditions

Location

Access and import/export

Disturbance
Describe the study conditions for field work, providing relevant parameters (e.g. temperature, rainfall).

State the location of the sampling or experiment, providing relevant parameters (e.g. latitude and longitude, elevation, water depth).

Describe the efforts you have made to access habitats and to collect and import/export your samples in a responsible manner and in compliance with local, national and international laws, noting any permits that were obtained (give the name of the issuing authority, the date of issue, and any identifying information).

\section{Reporting for specific materials, systems and methods}

We require information from authors about some types of materials, experimental systems and methods used in many studies. Here, indicate whether each material, system or method listed is relevant to your study. If you are not sure if a list item applies to your research, read the appropriate section before selecting a response.

\begin{tabular}{l|l} 
Materials \& experimental sys \\
\hline $\mathrm{n} / \mathrm{a}$ & Involved in the study \\
\hline & $\bigotimes$ Antibodies \\
\hline & $\square$ Eukaryotic cell lines \\
$\square$ & $\square$ Palaeontology \\
$\square$ & $\bigotimes$ Animals and other organisms \\
$\square$ & $\square$ Human research participants \\
$\square$ & $\square$ Clinical data
\end{tabular}

Methods $\mathrm{n} / \mathrm{a}$ Involved in the study

\ $\square$ chip-seq

$\square \bigotimes$ Flow cytometry

X| $\square$ MRI-based neuroimaging 
For Immunoblot analysis:

RSPO3 Polyclonal Antibody (Abclonal, A8389), 1:3000;

$\beta$-Actin (8H10D10) Mouse mAb (CST, \#3700), 1:5000;

For ChIP:

H3K4me3 antibody (CST, Cat\#9751), 1:500;

For hMeDIP:

5-Hydroxymethylcytosine (5-hmC) antibody (mAb) (Active Motif, Cat\#39999), 1:500;

For Immunofluorescence staining and Proximity Ligation Assay (PLA) :

anti-TET2 rabbit monoclonal antibody (CST, Cat\#36449) , 1:500;

anti- $\beta$-catenin mouse monoclonal antibody (Thermo Fisher Scientific, Cat\#13-8400), 1:500;

AF488-conjugated donkey anti-mouse IgG (Thermo Fisher Scientific, Cat\#A-21202), 1:2000;

AF594-conjugated goat anti-rabbit IgG (Thermo Fisher Scientific, Cat\#R37117), 1:2000.

FITC anti-mouse CD11b Antibody (clone M1/70), Reactivity: mouse, Application: Flow cytometry APC anti-mouse CD11b Antibody (clone M1/70), Reactivity: mouse, Application: Flow cytometry PE anti-mouse CD11b Antibody (clone M1/70), Reactivity: mouse, Application: Flow cytometry APC anti-mouse F4/80 Antibody (clone BM8), Reactivity: mouse, Application: Flow cytometry FITC anti-mouse F4/80 Antibody (clone BM8), Reactivity: mouse, Application: Flow cytometry PE anti-mouse F4/80 Antibody (clone BM8), Reactivity: mouse, Application: Flow cytometry APC anti-mouse CD64 Antibody (clone X54-5/7.1), Reactivity: mouse, Application: Flow cytometry FITC anti-mouse CD64 Antibody (clone X54-5/7.1), Reactivity: mouse, Application: Flow cytometry PE anti-mouse CD64 Antibody (clone X54-5/7.1), Reactivity: mouse, Application: Flow cytometry Alexa Fluor ${ }^{\circledast} 647$ anti-mouse CD64 Antibody (clone X54-5/7.1), Reactivity: mouse, Application: Flow cytometry APC anti-mouse CD206 Antibody (clone C068C2), Reactivity: mouse, Application: Flow cytometry Alexa Fluor 488 anti-mouse CD206 Antibody (clone C068C2), Reactivity: mouse, Application: Flow cytometry Alexa Fluor 700 anti-mouse CD206 Antibody (clone C068C2), Reactivity: mouse, Application: Flow cytometry PE anti-mouse CD206 Antibody (clone C068C2), Reactivity: mouse, Application: Flow cytometry PE anti-mouse CD86 Antibody (clone A17199A), Reactivity: mouse, Application: Flow cytometry Alexa Fluor 700 anti-mouse CD86 Antibody (clone A17199A), Reactivity: mouse, Application: Flow cytometry Pacific Blue anti-mouse CD86 Antibody (clone A17199A), Reactivity: mouse, Application: Flow cytometry APC anti-mouse CD301 Antibody (clone LOM-14), Reactivity: mouse, Application: Flow cytometry Pacific Blue anti-mouse CD301 Antibody (clone LOM-14), Reactivity: mouse, Application: Flow cytometry anti- Arginase1 Antibody (clone 658922) Reactivity: mouse, human Application: Flow cytometry APC anti-mouse CD80 Antibody (clone 16-10A1) Reactivity: mouse, human Application: Flow cytometry anti-mouse CD80 Antibody (clone 16-10A1) Reactivity: mouse, human Application: Flow cytometry PerCP/Cyanine5.5 anti-mouse CD80 Antibody (clone 16-10A1) Reactivity: mouse, human Application: Flow cytometry anti-mouse TNF $\alpha$ Antibody (clone MP6-XT22) Reactivity: mouse Application: Flow cytometry anti-NOS2 Antibody (clone 5C1B52) Reactivity: mouse, human Application: Flow cytometry PE anti-mouse IL-10 Antibody (clone JES5-16E3) Reactivity: mouse Application: Flow cytometry PE anti- $\beta$-Catenin Antibody (clone 12F7) Reactivity: mouse Application: Flow cytometry Alexa Fluor 488 anti- $\beta$-Catenin Antibody (clone 12F7) Reactivity: mouse Application: Flow cytometry anti-LGR4 Antibody (clone 852229) Reactivity: mouse, human Application: Flow cytometry anti-LGR5 Antibody (clone RD20) Reactivity: mouse Application: Flow cytometry anti-LGR6 Antibody (clone 918719R) Reactivity: mouse, human Application: Flow cytometry RSPO3 Polyclonal Antibody (clone AB_2554656) Reactivity: mouse, human Application: Flow cytometry Pacific Blue anti-mouse CD45 Antibody (clone 30-F11) Reactivity: mouse, Application: Flow cytometry PE/Cy5 anti-mouse CD45 Antibody (clone 30-F11) Reactivity: mouse, Application: Flow cytometry Brilliant Violet 605 Anti-mouse CD45 Antibody (clone 30-F11) Reactivity: mouse, Application: Flow cytometry PerCP anti-mouse Ly6G Antibody (clone 1A8) Reactivity: mouse, Application: Flow cytometry APC/Cy7 anti-mouse Siglec-F Antibody (clone E50-2440) Reactivity: mouse, Application: Flow cytometry PE anti-mouse Siglec-F Antibody (clone E50-2440) Reactivity: mouse, Application: Flow cytometry APC anti-mouse MERTK Antibody (clone 2B10C42) Reactivity: mouse, Application: Flow cytometry FITC anti-mouse MERTK Antibody (clone 2B10C42) Reactivity: mouse, Application: Flow cytometry PE anti-mouse MERTK Antibody (clone 2B10C42) Reactivity: mouse, Application: Flow cytometry 148Nd-anti-CD11b Antibody (clone M1/70) Reactivity: mouse, Application: Mass cytometry 159Tb-anti-F4/80 Antibody (clone BM8) Reactivity: mouse, Application: Mass cytometry 147Sm-anti-MerTK Antibody (clone 2B1042) Reactivity: mouse, Application: Mass cytometry 151Eu-anti-CD64 Antibody (clone X54-5/7.1) Reactivity: mouse, Application: Mass cytometry 141Pr-anti-Ly6G Antibody (clone 1A8) Reactivity: mouse, Application: Mass cytometry 162Dy-anti-Ly6C Antibody (clone HK1.4) Reactivity: mouse, Application: Mass cytometry 150Nd-anti-CD24 Antibody (clone M1/89) Reactivity: mouse, Application: Mass cytometry 169Tm-anti-CD206 Antibody (clone C068C2) Reactivity: mouse, Application: Mass cytometry 172Yb-anti-CD86 Antibody (clone GL1) Reactivity: mouse, Application: Mass cytometry 155Gd-anti-CD301 Antibody (clone AAH14811) Reactivity: mouse, Application: Mass cytometry 
149Sm-anti-CD19 Antibody (clone 6D5) Reactivity: mouse, Application: Mass cytometry 152Sm-anti-CD3 Antibody (clone 145-2C11) Reactivity: mouse, Application: Mass cytometry 170Er-anti-CD49 Antibody (clone HMa2) Reactivity: mouse, Application: Mass cytometry 176Yb-anti-SiglecF Antibody (clone E50-2440) Reactivity: mouse, Application: Mass cytometry 142Nd-anti-CD11c Antibody (clone N418) Reactivity: mouse, Application: Mass cytometry 156Gd-anti-Arginase 1 Antibody (clone P05089) Reactivity: mouse, Application: Mass cytometry 153Eu-anti-LGR4 Antibody (clone BBX-3A8) Reactivity: mouse, Application: Mass cytometry 165Ho-anti- $\beta$-catenin Antibody (clone D13A1) Reactivity: mouse, Application: Mass cytometry 154Sm-anti-TER119 Antibody (clone TER119) Reactivity: mouse, Application: Mass cytometry 158Gd-anti-IL-10 Antibody (clone JES5-16E3) Reactivity: mouse, Application: Mass cytometry 161Dy-anti-iNOS Antibody (clone CXNFT) Reactivity: mouse, Application: Mass cytometry 162Dy-anti-TNF Antibody (clone MP6-XT22) Reactivity: mouse, Application: Mass cytometry 164Dy-anti-CX3CR1 Antibody (clone SA011F11) Reactivity: mouse, Application: Mass cytometry 169Tm-anti-GFP Antibody (clone 5F12.4) Reactivity: mouse, Application: Mass cytometry 171Yb-anti-CD80 Antibody (clone 16-10A1) Reactivity: mouse, Application: Mass cytometry 209Bi-anti-I-A/I-E Antibody (clone 1M5/114.15.2) Reactivity: mouse, Application: Mass cytometry 89Y-anti-CD45 Antibody (clone 30-F11) Reactivity: mouse, Application: Mass cytometry 144Nd-anti-CD115 Antibody (clone AFS98) Reactivity: mouse, Application: Mass cytometry 145Nd-anti-CD69 Antibody (clone H1.2F3) Reactivity: mouse, Application: Mass cytometry 146Nd-anti-CCR2 Antibody (clone 475301R) Reactivity: mouse, Application: Mass cytometry 163Dy-anti-BST-2 Antibody (clone 44E9R) Reactivity: mouse, Application: Mass cytometry 168Er-anti-REMLa Antibody (clone Q9EP95) Reactivity: mouse, Application: Mass cytometry 173Yb-anti-CD103 Antibody (clone Q60677) Reactivity: mouse, Application: Mass cytometry RSPO3 Polyclonal Antibody (Abclonal, A8389), Reactivity: mouse, human, Application: western blot B-Actin Mouse mAb (clone, 8H10D10), Reactivity: mouse, human, Application: western blot H3K4me3 antibody (clone, C42D8), Reactivity: mouse, Application: ChIP

5-Hydroxymethylcytosine (5-hmC) antibody (mAb) , Reactivity: mouse, human, Application: hMeDiP anti-TET2 rabbit monoclonal antibody (clone, D6C7K), Reactivity: mouse, human, Application: IF anti- $\beta$-catenin mouse monoclonal antibody (clone, CAT-5H10), Reactivity: mouse, human, Application: IF

\section{Eukaryotic cell lines}

Policy information about cell lines

Cell line source(s)

State the source of each cell line used.

Authentication

Describe the authentication procedures for each cell line used OR declare that none of the cell lines used were authenticated.

Mycoplasma contamination

Confirm that all cell lines tested negative for mycoplasma contamination OR describe the results of the testing for mycoplasma contamination OR declare that the cell lines were not tested for mycoplasma contamination.

Commonly misidentified lines (See ICLAC register)

\section{Palaeontology}

Specimen provenance

Provide provenance information for specimens and describe permits that were obtained for the work (including the name of the issuing authority, the date of issue, and any identifying information).

Specimen deposition

Indicate where the specimens have been deposited to permit free access by other researchers.

Dating methods

If new dates are provided, describe how they were obtained (e.g. collection, storage, sample pretreatment and measurement), where they were obtained (i.e. lab name), the calibration program and the protocol for quality assurance OR state that no new dates are provided.

Tick this box to confirm that the raw and calibrated dates are available in the paper or in Supplementary Information.

\section{Animals and other organisms}

Policy information about studies involving animals; ARRIVE guidelines recommended for reporting animal research

Laboratory animals

Wild animals

Field-collected samples
8-12 weeks old age of both sex for the C57BL/6J, Rspo3fl/fl, Rspo3EC-/-, Ctnnb1fl/fl, Ctnnb1M $\phi-/-$, Tet2fl/fl, Tet2M $\phi-/$, and TCF/Lef:H2B-GFP mice strains were used.
Study did not involve wild animals.

The study did not involved Field-collected samples 


\section{Human research participants}

Policy information about studies involving human research participants

Population characteristics

Describe the covariate-relevant population characteristics of the human research participants (e.g. age, gender, genotypic

information, past and current diagnosis and treatment categories). If you filled out the behavioural \& social sciences study design questions and have nothing to add here, write "See above."

Recruitment

Describe how participants were recruited. Outline any potential self-selection bias or other biases that may be present and how these are likely to impact results.

Ethics oversight

Identify the organization(s) that approved the study protocol.

Note that full information on the approval of the study protocol must also be provided in the manuscript.

\section{Clinical data}

Policy information about clinical studies

All manuscripts should comply with the ICMJE guidelines for publication of clinical research and a completed CONSORT checklist must be included with all submissions.

Clinical trial registration

Provide the trial registration number from ClinicalTrials. gov or an equivalent agency.

Study protocol

Note where the full trial protocol can be accessed OR if not available, explain why.

Data collection

Describe the settings and locales of data collection, noting the time periods of recruitment and data collection.

Outcomes

Describe how you pre-defined primary and secondary outcome measures and how you assessed these measures.

ChIP-seq

\section{Data deposition}

Confirm that both raw and final processed data have been deposited in a public database such as GEO.

$\square$ Confirm that you have deposited or provided access to graph files (e.g. BED files) for the called peaks.

\section{Data access links}

May remain private before publication.

Files in database submission

Genome browser session (e.g. $\mathrm{UCSC}$ )

\section{Methodology}

\section{Replicates}

\section{Sequencing depth}

Antibodies

Peak calling parameters

Data quality

Software
For "Initial submission" or "Revised version" documents, provide reviewer access links. For your "Final submission" document, provide a link to the deposited data.

Provide a list of all files available in the database submission.

Provide a link to an anonymized genome browser session for "Initial submission" and "Revised version" documents only, to enable peer review. Write "no longer applicable" for "Final submission" documents. 


\section{Plots}

Confirm that:

\The axis labels state the marker and fluorochrome used (e.g. CD4-FITC).

W The axis scales are clearly visible. Include numbers along axes only for bottom left plot of group (a 'group' is an analysis of identical markers).

\All plots are contour plots with outliers or pseudocolor plots.

\A numerical value for number of cells or percentage (with statistics) is provided.

\section{Methodology}

Sample preparation

BMDMs were prepared by flushing and pipetting, filtered with $70 \mu \mathrm{m}$ strainer, and removal of red blood cells with RBC lysis buffer, and stained as depicted within the manuscript.

Single cell suspensions from lung tissues were cut into tiny pieces and digested with Collagenase/DNase I to at a shaking water bath for $30 \mathrm{~min}$, filtered with $70 \mu \mathrm{m}$ strainer, and removal of red blood cells with RBC lysis buffer, and stained as depicted within the manuscript.

Instrument

The CytoFLEX S Flow Cytometer (Beckman) was used for running the samples

Software

The Kaluza Analysis 2.1 software (Beckman) was used for analyzing the flow cytometry data.

Cell population abundance

Mouse lung IMs and AMs were isolated by Fluorescence-activated cell sorting (FACS) on the MoFlo Astrios cell sorter (Beckman Coulter) with the strategy described in the manuscript, with a purity of $>95 \%$ as verified by the indicated markers depicted within the manuscript.

Gating strategy

Gating strategy for BMDMs was depicted within the manuscript, briefly, BMDMs were gated with $\mathrm{CD} 11 \mathrm{~b}+\mathrm{F} 4 / 80+\mathrm{CD} 64+$, as well as shown in Supple. Fig. 1i.

$\bigotimes$ Tick this box to confirm that a figure exemplifying the gating strategy is provided in the Supplementary Information.

\section{Magnetic resonance imaging}

Experimental design

\section{Design type}

Design specifications

Behavioral performance measures

Acquisition

Imaging type(s)
Field strength
Sequence \& imaging parameters

Area of acquisition

Diffusion MRI

Used

\section{Preprocessing}

Preprocessing software

Normalization

Normalization template
Indicate task or resting state; event-related or block design.

Specify the number of blocks, trials or experimental units per session and/or subject, and specify the length of each trial or block (if trials are blocked) and interval between trials.

State number and/or type of variables recorded (e.g. correct button press, response time) and what statistics were used to establish that the subjects were performing the task as expected (e.g. mean, range, and/or standard deviation across subjects).

Specify: functional, structural, diffusion, perfusion.

Specify in Tesla

Specify the pulse sequence type (gradient echo, spin echo, etc.), imaging type (EPI, spiral, etc.), field of view, matrix size, slice thickness, orientation and TE/TR/flip angle.

State whether a whole brain scan was used OR define the area of acquisition, describing how the region was determined. Not used
Provide detail on software version and revision number and on specific parameters (model/functions, brain extraction, segmentation, smoothing kernel size, etc.).

If data were normalized/standardized, describe the approach(es): specify linear or non-linear and define image types used for transformation OR indicate that data were not normalized and explain rationale for lack of normalization.

Describe the template used for normalization/transformation, specifying subject space or group standardized space (e.g. original Talairach, MNI305, ICBM152) OR indicate that the data were not normalized. 
Noise and artifact removal

Volume censoring
Describe your procedure(s) for artifact and structured noise removal, specifying motion parameters, tissue signals and physiological signals (heart rate, respiration).

Define your software and/or method and criteria for volume censoring, and state the extent of such censoring.

Statistical modeling \& inference

Model type and settings

Specify type (mass univariate, multivariate, RSA, predictive, etc.) and describe essential details of the model at the first and second levels (e.g. fixed, random or mixed effects; drift or auto-correlation).

Effect(s) tested

Define precise effect in terms of the task or stimulus conditions instead of psychological concepts and indicate whether ANOVA or factorial designs were used.

Specify type of analysis:

Whole brain

ROI-based

Both

Statistic type for inference (See Eklund et al. 2016)

Specify voxel-wise or cluster-wise and report all relevant parameters for cluster-wise methods.

\section{Correction}

Describe the type of correction and how it is obtained for multiple comparisons (e.g. FWE, FDR, permutation or Monte (carlo).

Models \& analysis

\begin{tabular}{l|l} 
n/a & Involved in the study \\
\hline & $\square$ Functional and/or effective connectivity \\
\hline & $\square$ Graph analysis \\
\hline & $\square$ Multivariate modeling or predictive analysis
\end{tabular}

Functional and/or effective connectivity

Report the measures of dependence used and the model details (e.g. Pearson correlation, partial correlation, mutual information).

\section{Graph analysis}

Report the dependent variable and connectivity measure, specifying weighted graph or binarized graph, subject- or group-level, and the global and/or node summaries used (e.g. clustering coefficient, efficiency, etc.).

Multivariate modeling and predictive analysis

Specify independent variables, features extraction and dimension reduction, model, training and evaluation metrics. 